\title{
A neuronal model of classical conditioning
}

\author{
A. HARRY KLOPF \\ Air Force Wright Aeronautical Laboratories, Wright-Patterson Air Force Base, Ohio
}

\begin{abstract}
A neuronal model of classical conditioning is proposed. The model is most easily described by contrasting it with a still influential neuronal model first analyzed by Hebb (1949). It is proposed that the Hebbian model be modified in three ways to yield a model more in accordance with animal learning phenomena. First, instead of correlating pre- and postsynaptic levels of activity, changes in pre- and postsynaptic levels of activity should be correlated to determine the changes in synaptic efficacy that represent learning. Second, instead of correlating approximately simultaneous pre- and postsynaptic signals, earlier changes in presynaptic signals should be correlated with later changes in postsynaptic signals. Third, a change in the efficacy of a synapse should be proportional to the current efficacy of the synapse, accounting for the initial positive acceleration in the S-shaped acquisition curves observed in animal learning. The resulting model, termed a drivereinforcement model of single neuron function, suggests that nervous system activity can be understood in terms of two classes of neuronal signals: drives that are defined to be signal levels and reinforcers that are defined to be changes in signal levels. Defining drives and reinforcers in this way, in conjunction with the neuronal model, suggests a basis for a neurobiological theory of learning. The proposed neuronal model is an extension of the Sutton-Barto (1981) model, which in turn can be seen as a temporally refined extension of the Rescorla-Wagner (1972) model. It is shown that the proposed neuronal model predicts a wide range of classical conditioning phenomena, including delay and trace conditioning, conditioned and unconditioned stimulus duration and amplitude effects, partial reinforcement effects, interstimulus interval effects, secondorder conditioning, conditioned inhibition, extinction, reacquisition effects, backward conditioning, blocking, overshadowing, compound conditioning, and discriminative stimulus effects. The neuronal model also eliminates some inconsistencies with the experimental evidence that occur with the Rescorla-Wagner and Sutton-Barto models. Implications of the neuronal model for animal learning theory, connectionist and neural network modeling, artificial intelligence, adaptive control theory, and adaptive signal processing are discussed. It is concluded that real-time learning mechanisms that do not require evaluative feedback from the environment are fundamental to natural intelligence and may have implications for artificial intelligence. Experimental tests of the model are suggested.
\end{abstract}

Pavlov (1927/1960) and Hebb (1949) were among the first investigators to extensively analyze possible relationships between the behavior of whole animals and the behavior of single neurons. Building on Pavlov's experimental foundation, Hebb's theoretical analyses led him to a model of single neuron function that continues to be relevant to the theoretical and experimental issues of learning and memory. There had been earlier attempts to de-

This research was supported by the Life Sciences Directorate of the Air Force Office of Scientific Research under Task 2312 R1. Jim Morgan contributed substantially throughout the course of this research. In particular, I want to acknowledge many valuable discussions and the software Jim wrote for the computer simulations of the neuronal models. I am grateful to the following people for comments on an earlier draft of this article: Andy Barto, Diana Blazis, Jack Byrne, John Desmond, Bruce Edson, Mark Gluck, Chuck Hendrix, Joan Klopf, John Moore, Jim Morgan, Libby Patterson, Rick Ricart, and Rich Sutton. An abbreviated version of the results reported in this article will appear in a chapter entitled "Classical Conditioning Phenomena Predicted by a Drive-Reinforcement Model of Neuronal Function,"' to be published in Neural Models of Plasticity (J. H. Byrne \& W. O. Berry, Eds.), New York: Academic Press. My mailing address is AFWAL/AAAT-3, Wright-Patterson Air Force Base, OH 45433. velop such neuronal models. Among them were the models of Freud (1895/1964), Rashevsky (1938), and McCulloch and Pitts (1943/1965), but, to this day, the neuronal model proposed by Hebb has remained the most influential among theorists. Current theorists who have utilized variants of the Hebbian model include Anderson, Silverman, Ritz, and Jones (1977), Kohonen (1977), Grossberg (1982), Levy and Desmond (1985), Hopfield and Tank (1986), and Rolls (1987).

In this article, I will suggest several modifications to the Hebbian neuronal model. The modifications yield a model that will be shown to be more nearly in accord with animal learning phenomena that are observed experimentally. The model to be proposed is an extension of the Sutton-Barto (1981) model.

After defining the neuronal model, first qualitatively and then mathematically, I will show, by means of computer simulations, that the neuronal model predicts a wide range of classical conditioning phenomena. Then I will discuss the neuronal model in more general theoretical terms, with particular reference to the psychological notions of drives and reinforcers. My conclusion will be that the model offers a way of defining drives and reinforcers 
at a neuronal level such that a neurobiological basis is suggested for animal learning. In the theoretical context that the neuronal model provides, I will suggest that drives, in their most general sense, are simply signal levels in the nervous system, and that reinforcers, in their most general sense, are simply changes in signal levels. This seems too simple and, indeed, it is-but I hope to show that it is not that much too simple. I will attempt to make a case for drives and reinforcers being viewed, in their essence, as signal levels in the nervous system and as changes in signal levels, respectively. The result will be a theoretical framework based on what I propose to call a drive-reinforcement model of single neuron function.

\section{THE NEURONAL MODEL}

\section{Qualitative Description}

I will begin by defining the drive-reinforcement neuronal model in qualititive terms. It will be easiest to do this by contrasting the model with the Hebbian model. Hebb (1949) suggested that the efficacy of a plastic synapse increased whenever the synapse was active in conjunction with activity of the postsynaptic neuron. Thus, Hebb was proposing that learning (i.e., changes in the efficacy of synapses) was a function of correlations between approximately simultaneous pre- and postsynaptic levels of neuronal activity.

I wish to suggest three modifications to the Hebbian model:

1. Instead of correlating pre- and postsynaptic levels of activity, changes in presynaptic levels of activity should be correlated with changes in postsynaptic levels of activity. In other words, instead of correlating signal levels on the input and output sides of the neuron, the first derivatives of the input and output signal levels should be correlated.

2. Instead of correlating approximately simultaneous pre- and postsynaptic signal levels, earlier presynaptic signal levels should be correlated with later postsynaptic signal levels. More precisely and consistent with (1), earlier changes in presynaptic signal levels should be correlated with later changes in postsynaptic signal levels. Thus, sequentiality replaces simultaneity in the model. The interval between correlated changes in pre- and postsynaptic signal levels is suggested to range up to that of the maximum effective interstimulus interval in delay conditioning.

3. A change in the efficacy of a synapse should be proportional to the current efficacy of the synapse, accounting for the initial positive acceleration in the S-shaped acquisition curves observed in animal learning.

A refinement of the model will be noted now and discussed more fully later. The ability of the neuronal model to predict animal learning phenomena is improved if, instead of correlating positive and negative changes in neuronal inputs with changes in neuronal outputs, only positive changes in inputs are correlated with changes in outputs. To clarify this, positive changes in inputs refer to increases in the frequency of action potentials at a syn- apse, whether the synapse is excitatory or inhibitory. Negative changes in inputs refer to decreases in the frequency of action potentials at a synapse, whether the synapse is excitatory or inhibitory. Furthermore, the changes in frequencies of action potentials I'm referring to will be relatively abrupt, occurring within about a second or less. It is hypothesized that more gradual and long-term changes in the frequency of action potentials at a synapse do not trigger the neuronal learning mechanism.

After the neuronal model has been defined precisely and the results of computer simulations have been presented, it will be seen that this model of neuronal function bears the following relationship to models of whole-animal behavior. In general, changes in presynaptic frequencies of firing will reflect the onsets and offsets of conditioned stimuli. In general, changes in postsynaptic frequencies of firing will reflect increases or decreases in levels of drives (with drives being defined more broadly than has been customary in the past). In the case of the neuronal model, changes in the levels of drives (which will usually manifest as changes in postsynaptic frequencies of firing) will be associated with reinforcement. With regard to the behavior of whole animals, the notion that changes in drive levels constitute reinforcement has been a fundamental part of animal learning theory since the time of Hull (1943) and Mowrer (1960/1973). Here, I am taking the notion down to the level of the single neuron. Changes in signal levels, which play a fundamental role in the neuronal model being proposed, have long been recognized to be of importance. For example, Berlyne (1973) noted that

many recent theorists have been led from different starting points to the conclusion that hedonic value is dependent above all on changes in level of stimulation or level of activity. They include McClelland, Atkinson, Clark and Lowell (1953), Premack (1959), Helson (1964), and Fowler (1971) (p. 16).

Before concluding this introduction to the drivereinforcement neuronal model, it will be useful to note briefly how the model relates to earlier models from which it derives. The derivation and evolution of the model will be discussed more fully later. As has already been indicated, the drive-reinforcement model is an extension of the Sutton-Barto (1981) model. The Sutton-Barto model, in turn, can be viewed as a temporally refined extension of the Rescorla-Wagner (1972) model. I will show that the drive-reinforcement model eliminates some shortcomings of the Rescorla-Wagner and Sutton-Barto models. Both of the latter models predict strictly negatively accelerated acquisition or learning curves. The RescorlaWagner model also predicts extinction of conditioned inhibition. Consistent with the experimental evidence, it will be seen below that the drive-reinforcement model predicts (1) an acquisition curve that is initially positively accelerating and subsequently negatively accelerating, and (2) conditioned inhibition that does not extinguish. In addition, the drive-reinforcement model solves some problems with conditioned stimulus duration effects that arise in the case of the Sutton-Barto model. 


\section{Mathematical Specification}

The proposed neuronal model may be defined precisely as follows. The input-output relationship of a neuron will be modeled in a fashion that is customary among neural network modelers. Namely, it will be assumed that single neurons are forming sums of their weighted excitatory and inhibitory inputs, and then, if the sum equals or exceeds the threshold, that the neuron fires. Such a model of a neuron's input-output relationship can be based on the view that neuronal signals are binary (either a neuron fires or it does not) or on the view that neuronal signals are real-valued (reflecting some measure of the frequency of firing of neurons as a function of the amount by which the neuronal threshold is exceeded). Here, the latter view will be adopted. Neuronal input and output signals will be treated as frequencies. This approach to modeling neuronal input-output relationships is consistent with experimental evidence reviewed by Calvin (1975).

Mathematically, then, the neuronal input-output relationship may be specified as follows:

$$
y(t)=\sum_{i=1}^{n} w_{i}(t) x_{i}(t)-\theta
$$

where $y(t)$ is a measure of the postsynaptic frequency of firing at discrete time $t ; n$ is the number of synapses impinging on the neuron; $w_{i}(t)$ is the efficacy of the $i$ th synapse; $x_{i}(t)$ is a measure of the frequency of action potentials at the $i$ th synapse, and $\theta$ is the neuronal threshold. The synaptic efficacy, $w_{i}(t)$, can be positive or negative, corresponding to excitatory or inhibitory synapses, respectively. Also, $y(t)$ is bounded such that $y(t)$ is greater than or equal to zero and less than or equal to the maximal output frequency, $y^{\prime}(t)$, of the neuron. Negative values of $y(t)$ have no meaning because they would correspond to negative frequencies of firing.

To complete the mathematical specification of the neuronal model, the learning mechanism described earlier in qualitative terms remains to be presented. The learning mechanism may be specified as follows:

$$
\Delta w_{i}(t)=\Delta y(t) \sum_{j=1}^{\tau} c_{j}\left|w_{i}(t-j)\right| \Delta x_{i}(t-j)
$$

where $\Delta w_{i}(t)=w_{i}(t+1)-w_{i}(t), \Delta y(t)=y(t)-y(t-1)$, and $\Delta x_{i}(t-j)=x_{i}(t-j)-x_{i}(t-j-1)$. $\Delta w_{i}(t)$ represents the change in the efficacy of the $i$ th synapse at time $t$, yielding the adjusted or new efficacy of the synapse at time $t+1 . \Delta x_{i}(t-j)$ represents a change in the presynaptic signal level at time $t-j$, and $\Delta y(t)$ represents a change in the postsynaptic signal level at time $t . \tau$ is the longest interstimulus interval, measured in discrete time steps, over which delay conditioning is effective, and $c_{j}$ is an empirically established learning-rate constant that is proportional to the efficacy of conditioning when the interstimulus interval is $j$. The remaining symbols are defined as in Equation 1. Mathematical symbols used in this article are defined when introduced. In addition, a glossary listing the symbols and their definitions is located at the end of the article. A diagram of the neuron modeled by Equations 1 and 2 is shown in Figure 1.

Generally, in interpreting and working with Equation 2, I have adopted the following assumptions, consistent with what is known of learning involving the skeletal reflexes. I usually consider each discrete time step, $t$, to be equal to $.5 \mathrm{sec}$. This is a meaningful interval over which to obtain measures of the pre- and postsynaptic frequencies of firing, $x_{i}(t)$ and $y(t)$. Also, it is probably a reasonable interval of time with respect to the learning processes underlying changes in synaptic efficacy. For example, the optimal interstimulus interval for classically conditioning a skeletal reflex is nominally $.5 \mathrm{sec}$ (optimal interstimulus intervals vary from about 200 to $500 \mathrm{msec}$, depending on the species and the response system within the species; see review by Woody, 1982), and very little or no conditioning is observed with intervals approaching zero or exceeding $3 \mathrm{sec}$ (Frey \& Ross, 1968; McAllister, 1953; Russell, 1966; Moore \& Gormezano, 1977). Thus, in Equation 2, indexing starts with $j$ equal to 1 because $c_{0}$ is equal to zero, reflecting the fact that no conditioning is observed with an interstimulus interval of zero. $c_{1}$ is assigned the maximal value, reflecting the fact that $.5 \mathrm{sec}$ is (approximately) the optimal interstimulus interval. Then, $c_{j+1}$ is less than $c_{j}$ for the remaining $c$ values, reflecting the decreasing efficacy of conditioning as the interstimulus interval increases beyond $.5 \mathrm{sec} . \tau$ is normally set equal to 5 because when $j$ equals 6 (corresponding to an interstimulus interval of $3 \mathrm{sec}$ ), little or no conditioning would occur, so $c_{6}$ would be approximately equal to zero.

A lower bound is set on the absolute values of the synaptic weights, $w_{i}(t)$. The bound is near but not equal to zero because synaptic weights appear as factors on the right side of Equation 2. It can be seen that the learning mechanism would cease to yield changes in synaptic efficacy for any synapse whose efficacy reached zero; that is, $\Delta w_{i}(t)$ would henceforth always equal zero. A lower

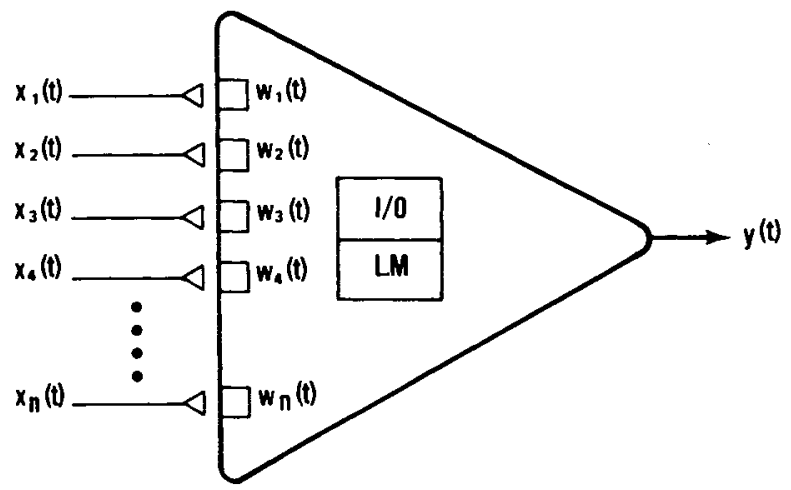

Figure 1. A model of a single neuron with $\boldsymbol{n}$ synapses. Presynaptic frequencies of firing are represented by $x_{t}(t)$, synaptic efficacies by $w_{i}(t)$, and the postsynaptic frequency of firing by $y(t)$. The inputoutput ( $(\mathbf{O} / \mathbf{O})$ relationship is specified by Equation 1 , and the learning mechanism (LM) is specified by Equation 2 in the text. 
bound on the absolute values of synaptic weights results in excitatory weights' always remaining excitatory (positive) and inhibitory weights' always remaining inhibitory (negative); that is, synaptic weights do not cross zero. This is consistent with the known physiology of synapses (Eccles, 1964). A nonzero lower bound on the efficacy of synapses is also consistent with evidence suggesting that potential conditioned stimuli are weakly connected to unconditioned responses prior to conditioning (Gould, 1986; Pavlov, 1927/1960; Schwartz, 1978). Also, a nonzero lower bound on the efficacy of synapses models the notion that a synapse must have some effect on the postsynaptic neuron in order for the postsynaptic learning mechanism to be triggered. That learning mechanisms are postsynaptic, at least in phylogenetically advanced organisms, has been well argued by McNaughton, Barnes, and Rao (1984). In the case of the mammalian central nervous system, Thompson, McCormick, et al. (1983) noted that what little evidence now exists is perhaps more consistent with the hypothesis of postsynaptic, rather than presynaptic, learning mechanisms.

In general, it is expected that the efficacy of synapses, $w_{i}(t)$, is variable and under the control of the neuronal learning mechanism. However, some synapses can be expected to have fixed weights, that is, weights that are innate and unchangeable. This may be true for many or most synapses in the autonomic nervous system. In the somatic nervous system, it is likely that many more synapses, and perhaps most, are variable or "plastic." In the case of the drive-reinforcement neuronal model, it will be assumed that synapses mediating conditioned stimuli have variable weights and that synapses mediating unconditioned stimuli have fixed weights. The innately specified synaptic weights that are assumed to mediate unconditioned stimuli are expected to reflect the evolutionary history of the organism.

Let us now consider what is happening in Equation 2. As the specification of the learning mechanism for the drive-reinforcement neuronal model, Equation 2 suggests how the efficacy of a synapse changes as a function of four factors: (1) learning-rate constants, $c_{j}$, that are assumed to be innate; (2) the absolute value, $\left|w_{i}(t-j)\right|$, of the efficacy of the synapse at time $t-j$, when the change in presynaptic level of activity occurred; (3) the change in presynaptic level of activity, $\Delta x_{i}(t-j)$; and (4) the change in postsynaptic level of activity, $\Delta y(t)$.

One way of visualizing either the Hebbian or the drivereinforcement learning mechanism is in terms of a temporal window that moves along the time line as learning occurs, changing the efficacy of synapses as it moves along. In the case of the Hebbian model, the learning mechanism employs a temporal window that is, in effect, only one time step wide. The learning mechanism moves along the time line, modifying the efficacy of synapses proportional to (1) a learning-rate constant, (2) the presynaptic level of activity, and (3) the postsynaptic level of activity. (The Hebbian model will be presented in mathematical form below.) In the case of the drive-reinforcement model, the learning mechanism employs a temporal window that is $\tau+1$ time steps wide. The learning mechanism moves along the time line modifying the efficacy of synapses proportional to (1) learning-rate constants, (2) the efficacy of synapses, (3) earlier changes in presynaptic levels of activity, and (4) later changes in postsynaptic levels of activity. It can be seen that the Hebbian learning mechanism correlates approximately simultaneous signal levels and the drivereinforcement learning mechanism correlates temporally separated derivatives of signal levels. (In the case of the drive-reinforcement model, I am not suggesting that a neuron would have to compute anything as refined as a first derivative. A first-order difference will suffice, as will be demonstrated below.) The differences in the behavior of the Hebbian and the drive-reinforcement learning mechanisms will be examined below with the results of computer simulations of both models.

\section{Properties of the Model}

The drive-reinforcement neuronal model suggests that neurons are learning to anticipate or predict the onsets and offsets of pulse trains. By pulse trains, I mean sequences or clusters of action potentials in axons. The model neuron learns to predict the onsets and offsets of pulse trains that represent unconditioned stimuli, utilizing the onsets of pulse trains that represent conditioned stimuli. This will become evident when the results of computer simulations are presented. It will be seen that the learning mechanism moves the onsets and offsets of pulse trains to earlier points in time. Fundamentally, the learning mechanism is a shaper of pulse trains. The efficacy of a synapse changes in a direction such that the neuron comes to anticipate the unconditioned response; that is, the conditioned stimulus comes to produce the conditioned response prior to the occurrence of the unconditioned stimulus and the unconditioned response. The way the drive-reinforcement neuronal learning mechanism shapes pulse trains is illustrated in Figure 2. Many investigators, including Pavlov (1927/1960), have pointed to the anticipatory or predictive nature of conditioning phenomena (see, e.g., Dickinson \& Mackintosh, 1978; Kamin, 1968, 1969; Rescorla \& Wagner, 1972; Sutton \& Barto, 1981].

\section{Refinement of the Model}

The drive-reinforcement neuronal learning mechanism, as defined by Equation 2, can be refined in a way that improves the model's ability to predict animal learning phenomena. The refinement, as briefly noted earlier, involves allowing only positive changes in presynaptic signal levels to trigger the neuronal learning mechanism. In other words, $\Delta x_{i}(t-j)$ must be greater than zero. If $\Delta x_{i}(t-j)$ is less than zero, it is then set equal to zero for the purpose of calculating $\Delta w_{i}(t)$ in Equation 2. 
(A) ONSETS

cs

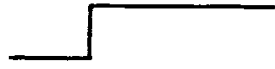

US
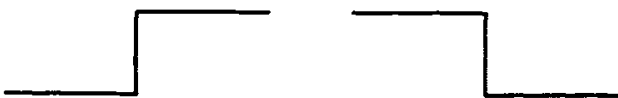

CR/UR: BEFORE LEARNING

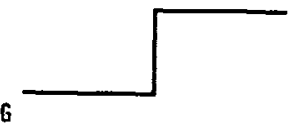

CR/UR: AFTER LEARNING

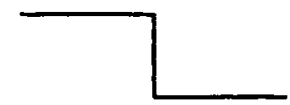

Figure 2. Examples of how the drive-reinforcement learning mechanism alters the onsets and ofisets of pulse trains for a single theoretical neuron. Panels $A$ and $B$ show the eflects of unconditioned stimulus onset and ofret, respectively. In each example, the conditioned stimulas (CS) is followed by an unconditioned stimulus (US), both of which represent presymaptic signals. The two presynaptic signols are aseumed to be medinted by separate synapes, with the CS-medinting synape hoving a variable eftcacy (weight) under the control of the neuromal learning mechanism. The conditioned and unconditioned response (CR and UR) before and atter learning (i.e., before and after a number of presentations of the CS-US pair) are shown below the woveforms for the CS and US pulse trains. The conditioned and unconditioned response (CR/UR) represents the postsymaptic frequency of firing of the neuron. In Panels A and $B$ it is seen that the onset and ofiset of firing, respectively, occur eariler in time after learning. Thus, in esch case, the neuron has learned to anticipate the unconditioned response by learning to start firing earlier (Panel A) or stop firing eartier (Panel B).

There is an intuitive basis for this refinement. A negative change in presynaptic signal level means that the presynaptic signal is falling away-that it is headed toward zero. If such a negative change in presynaptic signal level were to trigger the neuronal learning mechanism and possibly cause a synaptic weight to change, then a synaptic weight would have changed for a synapse that had just ceased to carry the signal that caused the change. That is to say, the relevant part of the signal on which the synaptic weight should operate would no longer be present. Some residual portion of the signal might still be present after the negative change in presynaptic signal level. However, the residual portion of the signal is not relevant, because it might have been there long before the negative change in presynaptic signal level and might be there long afterward. With the drive-reinforcement learning mechanism, only the dynamic part of the signal is relevant, as will be more clearly seen after the computer simulations are presented. This is not to suggest that a drive-reinforcement learning mechanism would preclude learning about negative changes in levels of stimuli. However, if such changes are to trigger a drivereinforcement learning mechanism, it is suggested that they would have to be, in effect, inverted, such that they would manifest in some part of the nervous system as positive changes in signal levels.

Allowing only positive changes in presynaptic signal levels to trigger the neuronal learning mechanism is part of a strategy of not changing a synaptic weight unless there is good reason to believe that the weight change will be useful. Such a strategy seems reasonable because, in a neural network, there is always the possibility that a synaptic weight change will interfere with or constitute overwriting of a previous weight change. Thus, weight changes are to be minimized.

The rationale offered above for refining the learning mechanism does not constitute a rigorous argument. The rationale may, however, provide some insight into why the refinement might make sense. Later, a more rigorous approach will be taken. It will be shown that a wide range of classical conditioning phenomena are predicted by the neuronal model when only positive changes in presynaptic signal levels are allowed to trigger the learning mechanism. Then it will be shown how the model's predictions deviate from the experimental evidence when both positive and negative changes in presynaptic signal levels can trigger changes in synaptic weights.

\section{Derivation and Evolution of the Drive-Reinforcement Model from Earlier Models}

Having defined the neuronal model in qualitative and mathematical terms, I will now describe the model's derivation and evolution from earlier neuronal models. The proposed neuronal learning mechanisms leading to the drive-reinforcement model will be portrayed in two ways: (1) by means of the sequence of critical events that have been hypothesized to lead to learning, and (2) by means of the equation that characterizes the learning mechanism. Since it is customary to number equations, I will also number the critical-event sequences so that I can refer to them later. To distinguish them from the equation numbers, an " $S$ " will be added as a prefix to the critical-event sequence numbers.

Hebb suggested that the sequence of critical events for learning was simple:

$$
x_{i}(t) \rightarrow y(t) \rightarrow \Delta w_{i}(t) .
$$

In other words, presynaptic activity, $x_{i}(t)$, followed directly by postsynaptic activity, $y(t)$, was hypothesized to result in a change, $\Delta w_{i}(t)$, in the efficacy of the associated synapse. (The convention adopted in this article is that when presynaptic activity, $x_{i}$, is a direct cause of postsynaptic activity, $y$, then $x_{i}$ and $y$ will have the same time step, $t$, associated with them.) The equation for the Hebbian learning mechanism may be written as follows:

$$
\Delta w_{i}(t)=c x_{i}(t) y(t),
$$

where $c$ is a learning-rate constant and the other symbols are as defined earlier.

Hebb's model is an example of a simple real-time learning mechanism. Real-time learning mechanisms empha- 
size the temporal association of signals: each critical event in the sequence leading to learning has a time of occurrence associated with it, and this time plays a fundamental role in the computations that yield changes in the efficacy of synapses. It should be noted that "real-time," in this context, does not mean continuous time as contrasted with discrete time; nor does it refer to a learning system's ability to accomplish its computations at a sufficient speed to keep pace with the environment within which it is embedded. Rather, a real-time learning mechanism, as defined here, is one for which the time of occurrence of each critical event in the sequence leading to learning is of fundamental importance with respect to the computations the learning mechanism is performing. Real-time learning mechanisms may be contrasted with non-real-time learning mechanisms such as the perceptron (Rosenblatt, 1962), adaline (Widrow, 1962), or back propagation (Le Cun, 1985; Parker, 1982, 1985; Rumelhart, Hinton, \& Williams, 1985, 1986; Werbos, 1974) learning mechanisms for which error signals follow system responses and only the order of the inputs, outputs, and error signals is important, not the exact time of occurrence of each signal, relative to the others. For additional discussions of realtime learning mechanism models, see Klopf $(1972,1975$, 1979, 1982, 1986), Moore and Stickney (1980), Sutton and Barto (1981, 1987), Wagner (1981), Grossberg (1982, 1987), Schmajuk and Moore (1985), Gelperin, Hopfield, and Tank (1985), Blazis, Desmond, Moore, and Berthier (1986), Tesauro (1986), and Donegan and Wagner (1987). Proposals for real-time models that give especially careful attention to neurobiological constraints are those of Hawkins and Kandel (1984) and Gluck and Thompson (1987).

Klopf $(1972,1982)$ proposed an extension to Hebb's model that introduced the notions of synaptic eligibility and reinforcement into real-time learning mechanisms, resulting in a neuronal model that emphasized sequential rather than simultaneous events. The following sequence of critical events was hypothesized to lead to learning:

$$
x_{i}(t-k) \rightarrow y(t-k) \rightarrow s(t) \rightarrow \Delta w_{i}(t),
$$

where $s(t)$ is the sum of the weighted inputs to the neuron at time $t$, and $k$ is the nominal interval of time required for a neuronal output to feed back and influence the neuronal input, the feedback occurring either through the remainder of the neural network or through the environment. The variable $s(t)$ represents the neuronal membrane potential. In this model, presynaptic and postsynaptic activity, $x_{i}(t-k)$ and $y(t-k)$, when they occur in conjunction, render a synapse eligible for modification. However, the efficacy of an eligible synapse does not change unless the subsequent membrane potential, $s(t)$, is nonzero, $s(t)$ functioning as a reinforcer that follows the eligibility computation. The equation for the learning mechanism is as follows:

$$
\Delta w_{i}(t)=c x_{i}(t-k) y(t-k) s(t) .
$$

In the context of real-time learning mechanisms, the notions of synaptic eligibility and reinforcement based on sequential rather than simultaneous events yielded a neuronal model that could make greater contact with the experimental evidence of classical and instrumental conditioning (Klopf, 1972, 1982). A further step was taken in this direction when Barto and Sutton (1981a) discovered that replacing $s(t)$ in Sequence S-2 above with $\Delta s(t)$ permitted the neuronal model to make much more substantial contact with classical conditioning phenomena. The resulting neuronal learning mechanism is described by the critical-event sequence

$$
x_{i}(t-k) \rightarrow y(t-k) \rightarrow \Delta s(t) \rightarrow \Delta w_{i}(t),
$$

where $\Delta s(t)=s(t)-s(t-1)$. The equation for the learning mechanism is

$$
\Delta w_{i}(t)=c x_{i}(t-k) y(t-k) \Delta s(t) .
$$

This form of learning mechanism led to a simplification. Barto and Sutton (1981a) found that the critical-event sequence (S-3) could be replaced with the following simpler sequence:

$$
x_{i}(t-k) \rightarrow \Delta y(t) \rightarrow \Delta w_{i}(t) .
$$

$\Delta y(t)$ in Sequence $S-4$ replaces $\Delta s(t)$ in Sequence $S-3$. This can be seen to be plausible in that $\Delta y(t)$ implies $\Delta s(t)$. However, proceeding from Sequence $S-3$ to Sequence $S-4$ involved the additional discovery that $y(t-k)$ in Sequence S-3 was not essential for predicting classical conditioning phenomena. The result was a neuronal model that can be specified by the following equation:

$$
\Delta w_{i}(t)=c x_{i}(t-k) \Delta y(t) .
$$

Actually, the form the model took in the computer simulations Sutton and Barto (1981) reported was as follows:

$$
\Delta w_{i}(t)=c \bar{x}_{i}(t) \Delta y(t),
$$

where

$$
\bar{x}_{i}(t)=\alpha \bar{x}_{i}(t-1)+x_{i}(t-1) .
$$

In Equation $8, \alpha$ is a positive constant. It can be seen that Equation 7 is similar in form to Equation 6, except that $x_{i}(t-k)$ is replaced by $\bar{x}_{i}(t), \bar{x}_{i}(t)$ represents an exponentially decaying trace of $x_{i}$ extending over a number of time steps.

It was at this point that neuronal modeling intersected strongly with the theoretical and experimental results of animal learning researchers such as Kamin (1968) and Rescorla and Wagner (1972). Sutton and Barto (1981) demonstrated that the model they proposed could be seen as a temporally refined extension of the Rescorla-Wagner (1972) model. Like the Rescorla-Wagner model, the Sutton-Barto model accounted for a variety of classical conditioning phenomena, including blocking, overshadowing, and conditioned inhibition. Here was what could be interpreted as a neuronal model (although Sutton and Barto did not insist on that interpretation) making predic- 
tions similar to those of a whole-animal model! The Sutton-Barto model represented a milestone in terms of the contact prospective neuronal models were making with the experimental evidence of animal learning (Barto \& Sutton, 1982; Blazis \& Moore, 1987; Moore et al., 1986; Sutton \& Barto, 1981).

However, the Sutton-Barto model still deviated from the experimental evidence in a number of significant respects. One problem was that the sensitivity of the model to conditioned stimulus durations caused the model to yield inaccurate predictions for a variety of conditioned stimulus-unconditioned stimulus configurations for which the conditioned stimulus and unconditioned stimulus overlapped significantly. The model also does not account for the initial positive acceleration in the S-shaped acquisition curves observed in classical conditioning.

One approach to correcting the problems of the SuttonBarto model has been to utilize a variant of the adaptive heuristic critic algorithm developed by Sutton (1984), and this has led to the temporal difference model proposed by Sutton and Barto (1987). Temporal difference models, as defined by Sutton and Barto (1987), utilize differences between temporally successive predictions as a basis for learning. Sutton (1987) noted that the earliest and most well known use of a temporal difference (TD) method or model was that used by Samuel $(1959 / 1963)$ in his checker-playing program. Other examples of TD methods or models include those used by Witten (1977), Sutton and Barto (1981), Booker (1982), Hampson (1983/1984), Sutton (1984), Gelperin et al. (1985), and Holland (1986). The drive-reinforcement neuronal model proposed in this article is an example of a TD model.

Variants of the adaptive heuristic critic model (Barto, Sutton, \& Anderson, 1983; Sutton, 1984) represent one approach to solving the problems of the Sutton-Barto model. In seeking to address these same problems, I have adopted an alternative approach that has led to the neuronal learning mechanism specified by Equation 2 . For this model, the hypothesized sequence of critical events leading to learning is as follows:

$$
\Delta x_{i}(t-j) \rightarrow \Delta y(t) \rightarrow \Delta w(t)
$$

where $j$ replaces $k$ and all of the critical events involve derivatives with respect to time. The variable $k$ was the time required for the neuron to receive feedback regarding its earlier output, $y(t-k) ; k$ reflected an instrumental conditioning orientation. The variable $j$ is simply an interstimulus interval that reflects a classical conditioning orientation. Barto and Sutton (1981a) had also considered using $\Delta x_{i}(t)$ instead of $x_{i}(t)$ in their learning mechanism but decided it was unworkable. I returned to this possibility of a differential learning mechanism, one that correlates earlier derivatives of inputs with later derivatives of outputs, and found a way to make it workable such that the problem with conditioned stimulus duration effects was eliminated. The class of differential learning mechanisms was independently discovered by Klopf (1986), coming from the directions of neuronal model- ing and animal learning, and by Kosko (1986), coming from the directions of philosophy and mathematics.

Sequence S-5 implies the following kind of learning mechanism:

$$
\Delta w_{i}(t)=c \Delta x_{i}(t-j) \Delta y(t)
$$

However, I have found that the most workable form of the learning mechanism involves adding multiple terms and multiple learning-rate constants to the right side of Equation 9, the terms and constants corresponding to a range of interstimulus intervals, $j$. Also, making $\Delta w_{i}(t)$ proportional to the absolute value of $w_{i}(t-j)$ allows the model to account for the initial positive acceleration in the acquisition curves of classical conditioning. These refinements led to the neuronal learning mechanism specified by Equation 2 and repeated here:

$$
\Delta w_{i}(t)=\Delta y(t) \sum_{j=1}^{\tau} c_{j}\left|w_{i}(t-j)\right| \Delta x_{i}(t-j)
$$

where $\Delta x_{i}(t-j)$ must be greater than or equal to zero; otherwise, $\Delta x_{i}(t-j)$ is set equal to zero for the purposes of Equation 10 . The resulting model predicts a wide range of classical conditioning phenomena, as will be demonstrated in the next section.

\section{CLASSICAL CONDITIONING: PREDICTIONS OF THE NEURONAL MODEL}

Classical conditioning phenomena are basic to learning. In this section, the drive-reinforcement neuronal model's predictions for classical conditioning phenomena will be examined by means of computer simulations of the model.

The neuronal model that was simulated is shown in Figure 3. The input-output (I/O) relationship assumed for the neuron was that of Equation 1. The neuronal learning

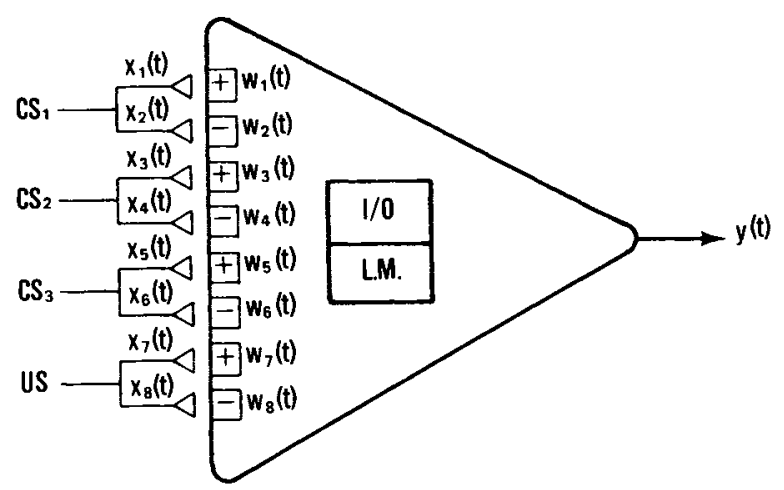

Figure 3. The drive-reinforcement neuronal model employed in the computer simulations. This is a specific example of the more general model shown in Figure 1. The description that was given in Figure 1 applies here. In addition, each CS and US is represented by an excitatory $(+)$ and an inhibitory $(-)$ synapse. The efícacies of synapses [i.e., the synaptic weights, $w_{i}(t)$ ] are variable (plastic) for synapses that mediate CSs and fixed (nonplastic) for synapses that mediate USs. 
mechanism (LM) was that of Equation 2 with the refinement noted earlier: Whenever $\Delta x_{i}(t-j)$ was less than zero, $\Delta x_{i}(t-j)$ was set equal to zero for the purpose of calculating $\Delta w_{i}(t)$. In the computer simulations, a conditioned stimulus (CS) or unconditioned stimulus (US) that was presented to the neuron had an amplitude that ranged between zero and one and a duration that was specified in terms of the times of stimulus onset and offset. In the figures showing results of the computer simulations, each CS-US configuration is graphed so the reader may see the relative amplitudes and durations of stimuli at a glance. (For exact values for any of the parameters for the computer simulations, the Appendix should be consulted.)

Each stimulus was presented to the simulated neuron through both an excitatory and an inhibitory synapse so that the neuronal learning mechanism had, for each input, both an excitatory and an inhibitory weight available for modification. The learning mechanism could then choose to modify one or the other weight, or both, in each time step. In the case of an actual (biological) neuron, if a CS is not represented by both excitatory and inhibitory synapses, the individual neuron will be constrained in terms of what classical conditioning phenomena it can manifest. It will be seen in the simulation below that, for a drive-reinforcement neuron, some classical conditioning phenomena require only excitatory plastic synapses and some require only inhibitory plastic synapses. The classical conditioning phenomena requiring both excitatory and inhibitory plastic synapses would have to emerge at a higher level if the individual neurons involved had their CSs represented by only excitatory or only inhibitory plastic synapses.

In the discussion that follows, a conditioned or unconditioned stimulus and the associated $x_{i}(t)$ in Figure 3 are identical. For example, $x_{1}(t)$ and $x_{2}(t)$ are one and the same as $C S_{1}$. The weights associated with the synapses carrying the unconditioned stimulus were fixed (nonplastic), and the remaining synaptic weights were variable (plastic).

The conditioned stimulus or unconditioned stimulus that is described should, perhaps, more properly be referred to as a neuronal conditioned stimulus or a neuronal unconditioned stimulus because it is the stimulus that is reaching the neuron, not the stimulus that is reaching the whole animal. However, for the sake of simplicity in the discussion, I will refer to these neuronal input signals as conditioned and unconditioned stimuli, or, simply, CSs and USs. Likewise, the output, $y(t)$, of the neuron would more properly be referred to as the neuronal conditioned or unconditioned response, but I will usually refer to the neuronal response as the conditioned response (CR) or unconditioned response (UR). Built into these terminological conventions is the assumption that stimuli and responses external to an animal's nervous system do not differ fundamentally in form from the way stimuli and responses are represented internal to the animal's nervous system. This assumption might not hold up well at higher, cognitive levels of function, but the assumption appears reasonable as a starting point for testing the ability of a neuronal model to predict fundamental learning phenomena.
Just as the range of $x_{i}(t)$ in the simulations was from zero to one, as was noted when the range of CS and US amplitudes was discussed, so the range of $y(t)$, the neuronal output, was from zero to one. Such a range serves to model a finite range of frequencies for neuronal inputs and outputs. Actual frequencies of biological neurons range up to several hundred spikes per second in the case of neocortical neurons firing for brief intervals (Lynch, Mountcastle, Talbot, \& Yin, 1977). Therefore, one could multiply the neuronal input and output amplitudes used in the simulations by, say, 300 if one desired to see more realistic numbers. However, for the purposes of the simulations to be reported, it is the relative magnitudes of the parameters that are important, not the absolute magnitudes.

The number of synapses impinging on the simulated neuron is eight, as is indicated in Figure 3 . This corresponds to three possible CSs and one US. The absolute values of the plastic synaptic weights mediating the CSs have a lower bound of 0.1 and, when the simulations began, these excitatory and inhibitory weights were set at plus and minus 0.1 , respectively. (For exceptions to this statement, see the Appendix; in some simulations, inhibitory synaptic weights were set equal to zero because they did not play a significant role and it simplified the graphs). The neuronal threshold was set at zero because, at higher values of the neuronal threshold, the form of the model's predictions did not change. The only effect of higher thresholds was that more trials were required for the synaptic weights to reach their asy mptotic values. For the learning mechanism, the learning-rate constants, $c_{1}$ through $c_{5}$, were set at values such that $c_{j}>c_{j+1}$. As noted earlier, this is reasonable if one views each time step as being equivalent to $.5 \mathrm{sec}$, because then $c_{1}$ is maximal, corresponding to a nominal optimal interstimulus interval of $.5 \mathrm{sec}$. Successive $c$ values then decrease as the interstimulus interval increases. As also noted earlier, $c_{0}$ and $c_{6}$ were set equal to zero, corresponding to interstimulus intervals of 0 and $3 \mathrm{sec}$, respectively. Thus, in the simulations, $j$ ranged from 1 to 5 ; that is, $\tau$ was set equal to 5 .

What follows are the results of computer simulations of the drive-reinforcement neuronal model for a variety of CS-US configurations. The predictions of the model are examined for delay and trace conditioning, CS and US duration and amplitude effects, partial reinforcement effects, interstimulus interval effects, second-order conditioning, conditioned inhibition, extinction, reacquisition effects, backward conditioning, blocking, overshadowing, compound conditioning, and discriminative stimulus effects.

During a simulation, the CS-US configuration was presented once in each trial. The values of the synaptic weights at the end of each trial were recorded and plotted as a function of the trial number. These graphs of synaptic weights versus trials are shown in the figures accompanying the discussion below. In addition, in each figure, the CS-US configuration is graphed along with the response of the neuron during the last trial. The neuronal response is labeled "Y," designating a plot of $y(t)$ for the last trial of the simulation. The definition of a trial should be noted. The 
CS-US configuration, or what is referred to in the figures as the "stimulus configuration," defines a trial. Thus, the graphed stimulus configurations in the figures are intended to show not only relative times of onset and offset along with amplitudes of stimuli, but also the number of times a stimulus was presented during a trial. What will be seen in the figures is that the behavior of the synaptic weights, as predicted by the drive-reinforcement neuronal model, mirrors the observed behavior of animals as they are learning during classical conditioning experiments.

Before discussing the individual simulations, two remarks are in order regarding the graphs of synaptic weights versus trials. Any synaptic weight that played a significant role for the conditioning phenomenon being discussed is shown in the accompanying graph. To simplify the graphs, any synaptic weight that played no significant role (typically meaning that the neuronal learning mechanism did not alter the weight at ali during the simulation) is not shown. Also, data points for the synaptic weight values at the end of each trial are not shown on the graphs, because the resulting density of the data points would be excessive and because the data points fall exactly on the (theoretical) curves that have been drawn.

\section{Delay Conditioning}

Delay conditioning is defined such that CS onset precedes US onset and CS offset occurs at the same time as or after US onset. An example is the well-known Pavlovian experiment in which a bell (the CS) is paired with food (the US). The observed result in such experiments is that conditioned excitation develops. The bell becomes excitatory with respect to the salivary gland. In addition, it is observed that the amount of salivation in response to the bell alone (measured with occasional test probes) increases with increasing numbers of trials, such that an S-shaped, or sigmoid, curve results when the amount of salivation is plotted versus the trial number. That is to say, the amount of salivation in response to the bell alone, as a function of trials, positively accelerates initially and then negatively accelerates as an asymptotic level of conditioning is approached (Pavlov, 1927/1960). Spence (1956) has observed that the acquisition curves of classical conditioning are always $\mathrm{S}$-shaped, if the experiments are done carefully enough to capture the initial positive acceleration and the later negative acceleration. For example, Spence (1956) stated that acquisition curves that

do not exhibit an initial, positively accelerated phase do not do so either because they do not start at zero level of conditioning or because the conditioning is so rapid that the period of initial acceleration is too brief to be revealed except by very small groups or blocks of trials. (pp. 68 and 70)

Figure 4 shows the predicted acquisition curves of three neuronal models for delay conditioning. In Figure $4 a$, the results of a simulation of the model proposed by Hebb (1949) are shown. For the Hebbian model, the input-output relationship is the same as for the drive-reinforcement model and is, therefore, specified by Equation 1. The Hebbian learning mechanism has already been noted and is specified by Equation 3. It can be seen in Figure 4a that if a Hebbian neuron were driving the salivary gland, the amount of saliva produced in response to the bell alone as a function of the number of trials would exhibit an essentially linear relationship, because the excitatory synaptic weight associated with the CS varies in an essentially linear fashion with the trial number. Also, it may be noted that the Hebbian learning mechanism does not yield an asymptotic synaptic weight value but, rather, continues to increase the synaptic weight indefinitely or, of course, until an upper bound is reached.

In Figure 4b, the results of a simulation of the SuttonBarto (1981) model are shown. The Sutton-Barto learning mechanism was specified earlier in Equations 7 and 8. The model's input-output relationship is that of Equation 1. The model is seen to predict a negatively accelerated acquisition curve in that the excitatory synaptic weight associated with the CS negatively accelerates with increasing numbers of trials. It may be noted that the Rescorla-Wagner (1972) model also predicts a negatively accelerated acquisition curve, as have earlier whole-animal models (see, e.g., Estes, 1950).

In Figure $4 c$, the results of a simulation of the drivereinforcement model are shown. The model is seen to predict an S-shaped acqusition curve: conditioned excitation develops, first through a positively accelerating phase and then through a negatively accelerating phase. The drive-reinforcement model is thus seen to be consistent with this aspect of the experimental evidence of delay conditioning.

Some reasons why the drive-reinforcement model yields an S-shaped acquisition curve may be noted. The initial positive acceleration is due to the efficacy of the relevant synapse's occurring as a factor on the right side of Equation 2 . Thus, as the learning mechanism increases the efficacy of the synapse, the future rate of change of the efficacy of the synapse is also caused to increase. With continued conditioning, another process comes to dominate, yielding the eventual negative acceleration in the acquisition curve. The negative acceleration is due to the decrease of $\Delta y(t)$ with continued conditioning. In effect, with conditioning, $\Delta y(t)$ moves to an earlier point in time, becoming $\Delta y(t-j)$, where $j$ is the interstimulus interval. Thus, throughout the conditioning process, increasing values of $w_{i}(t-j)$ are competing with decreasing values of $\Delta y(t)$ in Equation 2. Rapidly increasing values of $w_{i}(t-j)$ prevail initially and rapidly decreasing values of $\Delta y(t)$ prevail later, yielding the respective positive and negative accelerations in the acquisition curve.

\section{CS and US Duration Effects}

A careful reader may note that, in Figure 4, the same CS-US configuration is not used for the simulation of each of the models. The CS offset of the Hebbian model coincides with the offset of the US, whereas the CS offsets of the Sutton-Barto and drive-reinforcement models occur at the time of US onset. I chose those particular CS-US configurations because, otherwise, the Hebbian and Sutton-Barto models would not have predicted the development of condi- 
(a) HEBBIAN MODEL

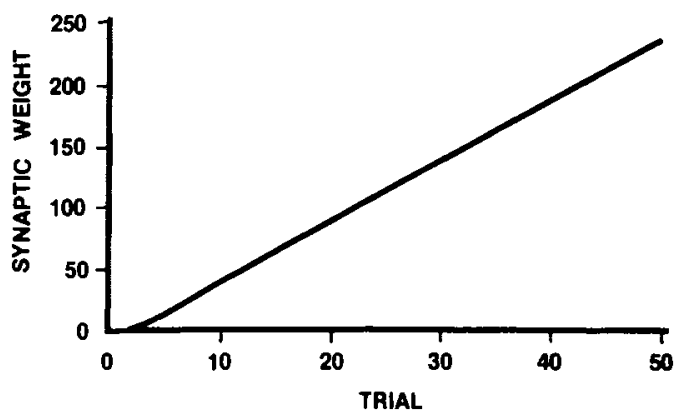

(b) SUTTON-BARTO MODEL

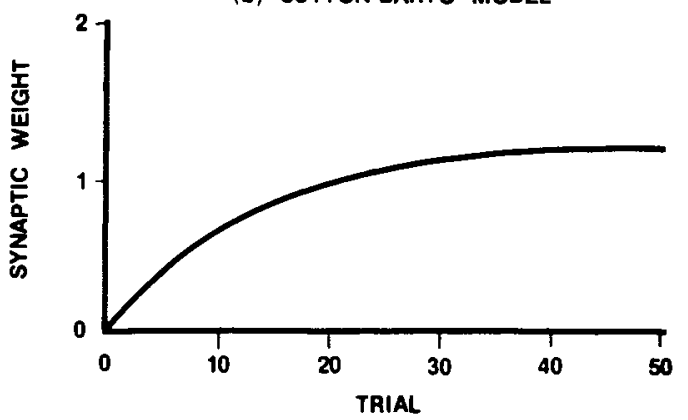

(c) DRIVE-REINFORCEMENT MODEL

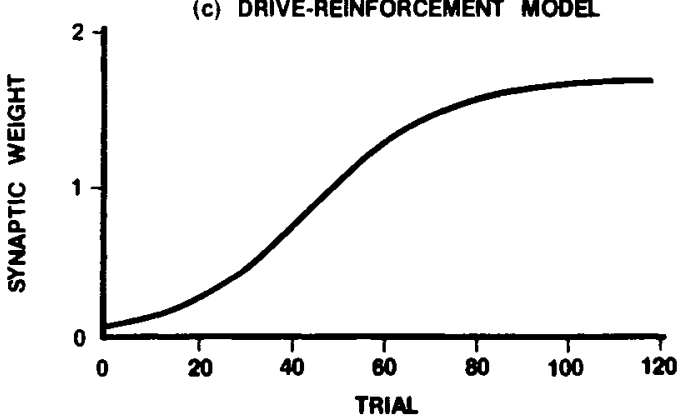

STIMULUS

CONFIGURATION

AND RESPONSE:
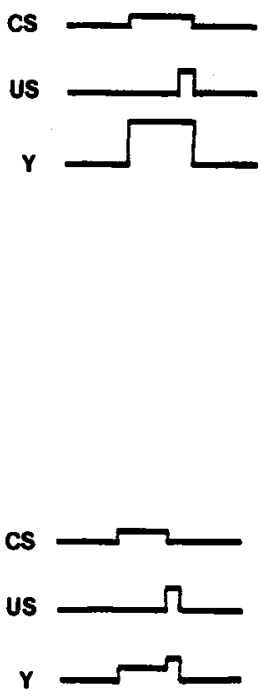

Figure 4. Results of simulated delay conditioning experiments with (a) Hebbian, (b) Sutton-Barto, and (c) drive-reinforcement models. The Hebbian model yields an essentially linear acquisition curve. The Sutton-Barto model yields a negatively accelerated acquisition curve. Consistent with the experimental evidence, the drivereinforcement model yields an S-shaped acquisition curve. (See text and Appendix for details.)

tioned excitation. Both of these models are sensitive to CS durations in a way that is not consistent with the experimental evidence, with the models predicting no conditioning or conditioned inhibition for some CS-US configurations that, experimentally, are known to yield conditioned excitation. The effect of CS duration is examined systematically in Figure 5, with each model's predictions shown for the same set of three CS-US configurations. I will specify how the three CS-US configurations differ and then discuss each model's predictions for each of the three configurations.

In Figure 5, CS 1 offset occurs at the time of US onset, $\mathrm{CS}_{2}$ offset occurs at the time of US offset, and $\mathrm{CS}_{3}$ offset occurs one time step after US offset. Experimentally, it is known that conditioned excitation (corresponding in the neuronal models to the growth of positive synaptic weights) is observed in all three cases. In general, the efficacy of delay conditioning is a strong function of the time of CS onset and is relatively independent of CS duration (Kamin, 1965).

In Figure 5a, it is seen that the Hebbian model predicts conditioned excitation for $\mathbf{C S}_{\mathbf{2}}$ and $\mathrm{CS}_{3}$ but not for $\mathrm{CS}_{\mathbf{1}}$. In Figure 5b, the Sutton-Barto model predicts conditioned excitation for $\mathrm{CS}_{1}$ and strong conditioned inhibition for $\mathrm{CS}_{2}$ and $\mathrm{CS}_{3}$. In Figure $5 \mathrm{c}$, consistent with the experimental evidence, the drive-reinforcement model predicts conditioned excitation for all three CSs and, in each case, predicts an Sshaped acquisition curve. 


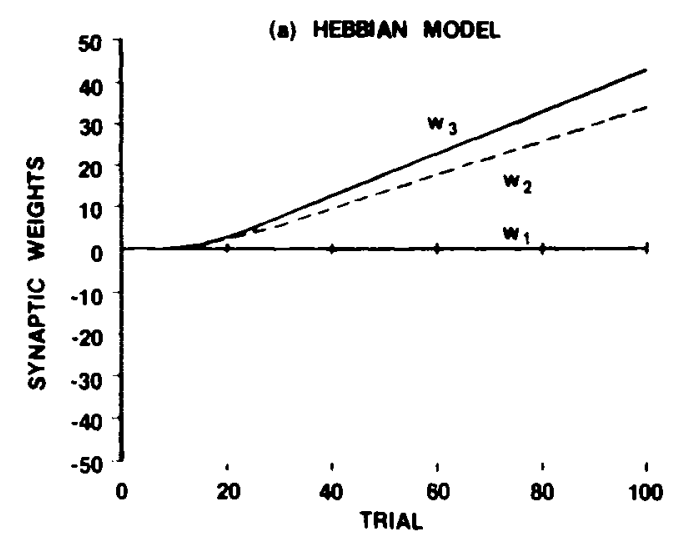

\section{STIMULUS COMFIGUAATION} AND RESPONSE:

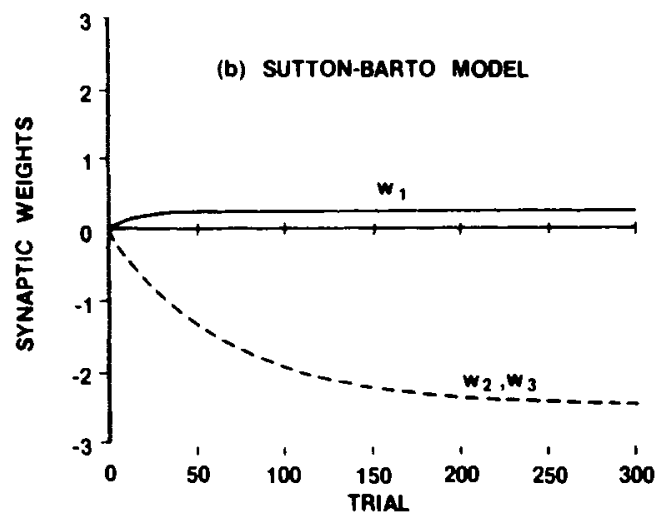

STIMULUS CONFIGUAATION AND RESPONSE:
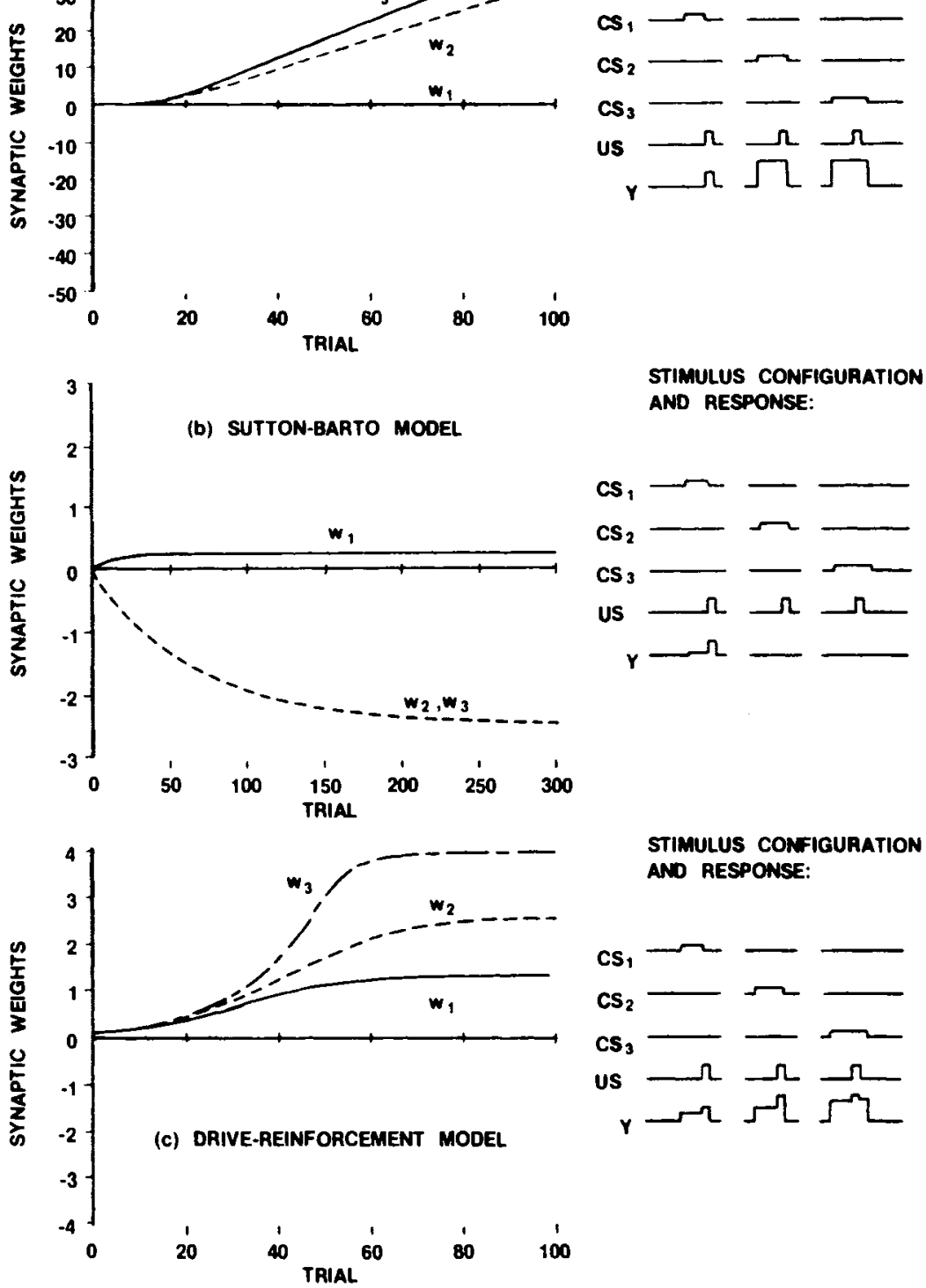

STIMULUS CONFIGURATION ANO RESPONSE:

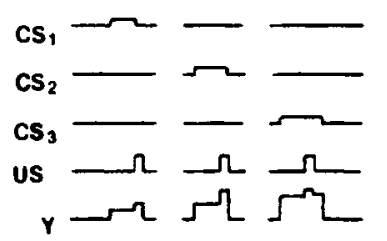

Figure 5. Results of simulated delay conditioning experiments with (a) Hebbian, (b) Sutton-Barto, and (c) drive-reinforcement models. The effect of CS duration is examined. $\mathrm{CS}_{1}$ offset occurs at the time of US onset. $\mathrm{CS}_{2}$ offset oocurs at the time of US offset. $\mathrm{CS}_{3}$ offset occurs after US offset. The Hebbian model yields conditioned excitation for $\mathbf{C S}_{2}$ and $\mathrm{CS}_{3}$ and no conditioning for $\mathrm{CS}_{1}$. The Sutton-Barto model yields conditioned excitation for $\mathrm{CS}_{1}$ and conditioned inhibition for $\mathrm{CS}_{2}$ and $\mathrm{CS}_{3}$. Consistent with the experimental evidence, the drive-reinforcement model yields conditioned excitation for $\mathrm{CS}_{1}, \mathrm{CS}_{2}$, and $\mathrm{CS}_{3}$. (See text and Appendix for details.)

In Figure 5c, more detailed aspects of the drivereinforcement model's predictions may be noted. For example, the model predicts a particular ranking of CSs in terms of initial rate of conditioning and asymptotic synaptic weight value as a function of $\mathrm{CS}$ duration. The experimental literature does not, at this point, permit the accuracy of these more detailed predictions to be assessed. Furthermore, whole-animal data may be insufficient to test these predictions, in that higher level attention mechanisms may play a significant role when CS durations are extended beyond the US (Ayres, Albert, \& Bombace, 1987). Experiments at the level of the single neuron may be required to test these predictions.

Regarding the effects of US duration, the drivereinforcement model predicts increasing rates of conditioning and higher asymptotic levels as the US duration in- 
STIMULUS CONFIGURATION AND RESPONSE:
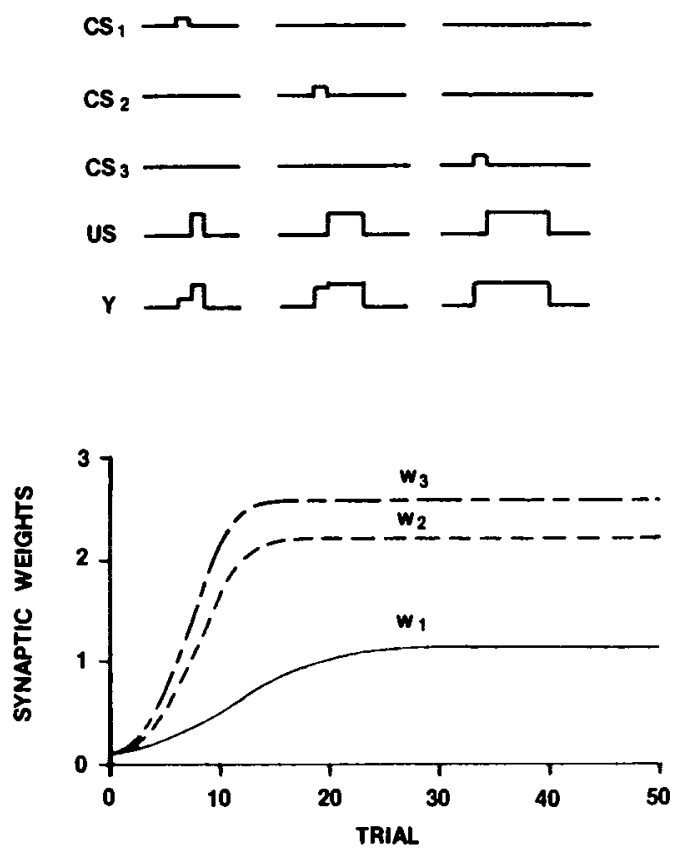

Figure 6. The drive-reinforcement model's predictions of the effects of US duration. Consistent with the experimental evidence, as the US duration increases, the excitatory synaptic weights associated with the reinforced CSs increase more rapidly and reach a higher asymptotic level. (See text and Appendix for details.)

creases (see Figure 6), consistent with the experimental evidence (Ashton, Bitgood, \& Moore, 1969; Gormezano, Kehoe, \& Marshall, 1983).

Thus far, the drive-reinforcement model's predictions have been demonstrated to be accurate for three categories of classical conditioning phenomena: (1) the form of the acquisition curve in delay conditioning, (2) relative insensitivity to CS duration, and (3) US duration effects. The predictions of the model for a variety of other CS-US configurations will now be examined. The predictions of the Hebbian and Sutton-Barto models for these CS-US configurations will not be shown, but it should be noted that the Hebbian model's predictions frequently deviate substantially from experimentally observed behavior, examples of this having already been seen in Figures 4 and 5. (Of course, it remains a theoretical possibility that biological neurons are Hebbian and that classical conditioning phenomena are emergent, resulting from the interactions of perhaps large numbers of Hebbian neurons. Experimental tests to be discussed later will be required to resolve this question.) The predictions of the Sutton-Barto model are similar to those of the drive-reinforcement model if one is careful, in the case of the Sutton-Barto model, not to use substantially overlapping CSs and USs and accepting that the Sutton-Barto model's predicted acquisition curves are not S-shaped.

\section{CS and US Amplitude Effects}

It is known that faster conditioning results as the intensity of the CS increases (Pavlov, 1927/1960; see review by Moore \& Gormezano, 1977). As can be seen in Figure 7, the drive-reinforcement model predicts this relationship. Shown in Figure 7 are CSs of three different amplitudes, each being reinforced by a US of the same amplitude. The predicted rate of conditioning is seen to increase as the amplitude or intensity of the CS increases. For the three CSs, the rank ordering of the asymptotic values of the synaptic weights is the reverse of the rank ordering of the rates of acquisition, because a low-amplitude CS requires a larger asymptotic synaptic weight to yield the same eventual CR amplitude as can be obtained with a high-amplitude CS and a lower asymptotic synaptic weight.

Regarding US amplitude effects, Moore and Gormezano (1977) noted that "within limits, the rate of acquisition and level of performance of a CR are increasing functions of the intensity of the US"' (p. 115). This is predicted by the drivereinforcement model, as can be seen in Figure 8, in which three identical CSs are being reinforced by USs of decreasing amplitude. It can be seen that both the rate of acquisition

STIMULUS CONFIGURATION AND RESPONSE:
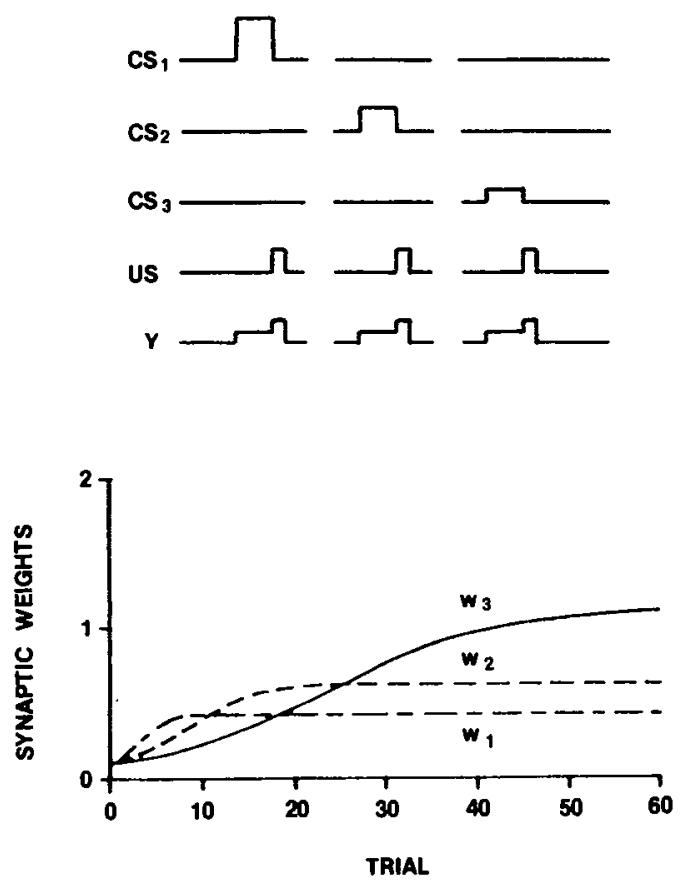

Figure 7. The drive-reinforcement model's predictions of the effects of CS amplitude. Consistent with the experimental evidence, as the CS amplitude decreases, the rate of growth of the excitatory synaptic weights associated with the reinforced CSs decreases. Asymptotic excitatory synaptic weight values vary inversely with CS amplitude because a lower $C S$ amplitude requires a higher excitatory asymptotic synaptic weight value to yield a CR amplitude equal to the UR amplitude. (See text and Appendix for details.) 
STIMULUS CONFIGURATION AND RESPONSE:
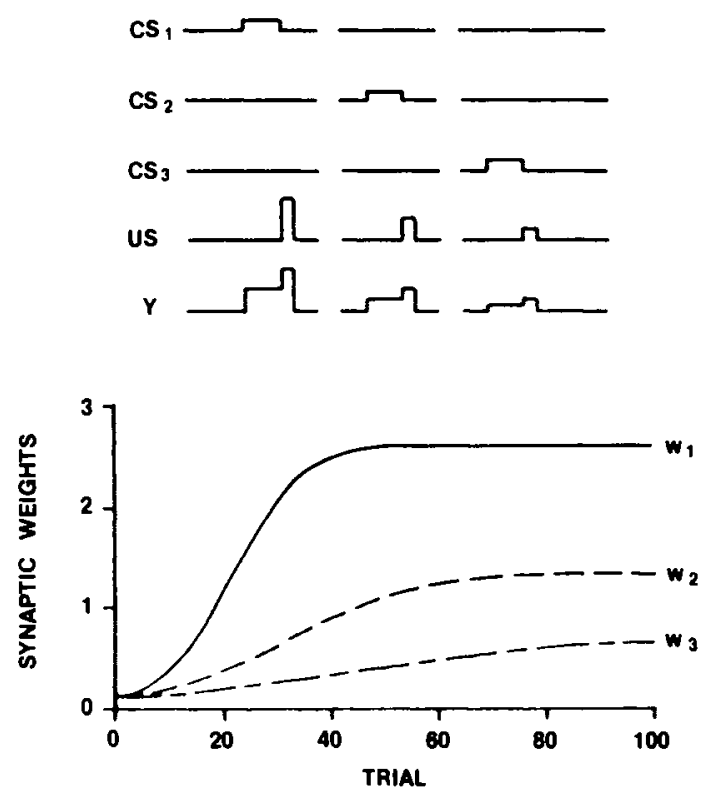

Figure 8. The drive-reinforcement model's predictions of the effects of US amplitude. Consistent with the experimental evidence, as the US amplitude decreases, the rates of growth and asymptotic values of excitatory synaptic weights associated with the reinforced CSs decrease. (See text and Appendix for details.)

and the asymptotic weight value decrease as the US amplitude decreases.

\section{CS Preexposure Effects}

CS preexposure refers to nonreinforced presentations of a CS prior to reinforced presentations. The observed result is that CS preexposure retards subsequent acquisition of the conditioned response when reinforced presentations of the CS begin, although the experimental evidence also suggests that the preexposed CS does not become inhibitory (see review by Flaherty, 1985, who cites, e.g., Rescorla, 1971a, Reiss \& Wagner, 1972, and Solomon, Brennan, \& Moore, 1974). As Flaherty (1985) notes, one possible explanation for CS preexposure effects is that the animal may, during the nonreinforced CS presentations, learn not to attend to the stimulus. If this is the case, CS preexposure effects would not be predicted by a neuronal model. Rather, such effects would require network-level considerations for their prediction. The related subject of US preexposure effects will be discussed below, when the phenomenon of blocking is considered.

\section{Partial Reinforcement Effects}

In the case of partial reinforcement, a CS is not always followed by a US. This can be contrasted with continuous reinforcement, where the US always follows the CS. The observed result of partial reinforcement is a reduced rate of conditioning and sometimes a reduced asymptotic level of responding (Gormezanc et al., 1983) relative to the rates and asymptotic levels observed for continuous reinforcement. The drive-reinforcement model's predictions are consistent with this, as can be seen in Figure 9, where $\mathrm{CS}_{\mathbf{1}}$ is reinforced $100 \%$ of the time, $\mathrm{CS}_{2}$ is reinforced $50 \%$ of the time, and $\mathrm{CS}_{3}$ is reinforced $25 \%$ of the time. In Figure 9 , it can be seen that rates of acquisition and asymptotic weight values are predicted to decrease as the percent reinforcement decreases.

\section{Trace Conditioning}

Trace conditioning is an experimental procedure in which CS offset precedes US onset. The time between CS offset and US onset is termed the trace interval. In general, the longer the trace interval, the lower the rate of acquisition and the lower the asymptotic level of conditioning (see Flaherty, 1985, for a review of the experimental evidence). The drive-reinforcement model predicts these relationships, as can be seen in the three CS-US configurations shown in Figure 10. It can be seen that increasing trace intervals yielded both lower rates of acquisition and lower asymptotic synaptic weight levels. In terms of the drivereinforcement model's dynamics, some reasons that trace conditioning is less effective than delay conditioning are that the $\Delta x$ that occurs at CS onset is paired not only with the positive $\Delta y$ of US onset, but also with the negative $\Delta y$ of CS offset and, furthermore, the interstimulus interval for the negative $\Delta y$ has a larger learning-rate constant associated with it than does the interstimulus interval for the positive $\Delta y$.

STIMULUS CONFIGURATION AND RESPONSE:
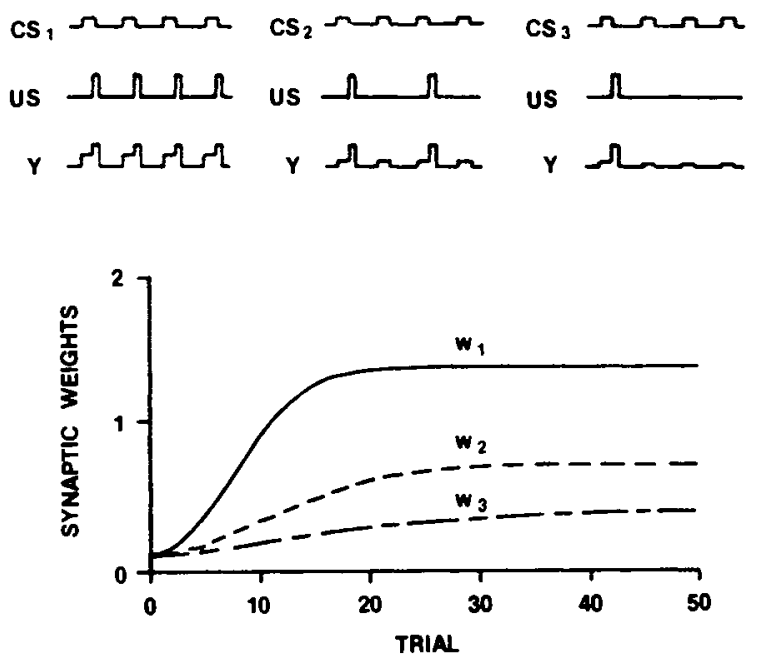

Figure 9. The drive-reinforcement model's predictions of the effects of partial reinforcement. Consistent with the experimental evidence, as the fraction of CSs that are reinforced decreases, so does the rate of growth of excitatory synaptic weights associated with the reinforced CSs. The drive-reinforcement model also predicts lower asymptotic excitatory synaptic weight values as the percentage of reinforced CSs decreases, an effect that is consistent with some partial reinforcement studies. (See text and Appendix for details.) 
STIMULUS CONFIGURATION AND RESPONSE:
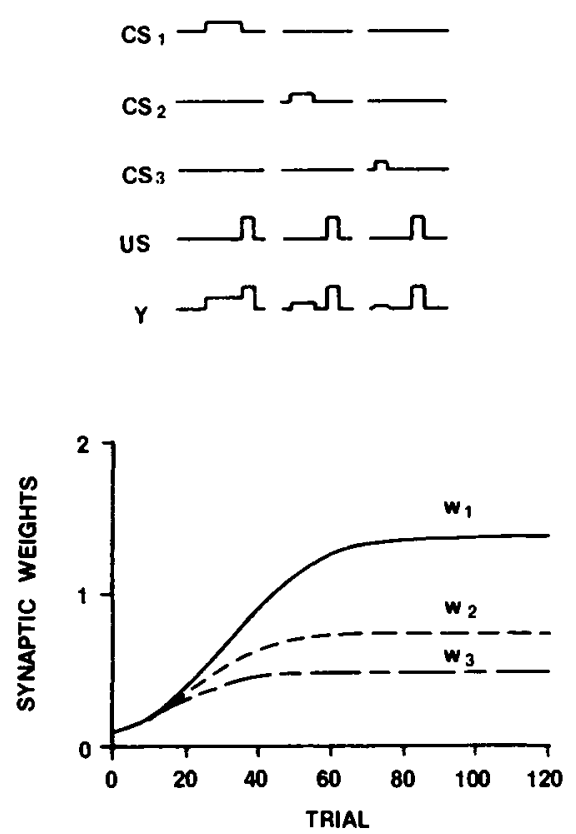

Figure 10. The drive-reinforcement model's predictions of the effects of trace conditioning. Consistent with the experimental evidence, as the trace interval increases, the rates of growth and asymptotic values of the excitatory synaptic weights associated with the reinforced CSs decrease. (See text and Appendix for details.)

\section{Interstimulus Interval Effects}

The predictions of the drive-reinforcement model for a variety of interstimulus intervals in delay conditioning are shown in Figure 11. The interstimulus interval is defined to be the time between CS and US onsets. In the case of $\mathrm{CS}_{1}$ in Figure 11, CS and US onsets are simultaneous. This CS-US configuration is an example of what is referred to as simultaneous conditioning. Citing Pavlov (1927/1960), as well as Smith, Coleman, and Gormezano (1969), Flaherty (1985) notes that "little or no conditioning occurs with simultaneous CS and US onset"' (p. 69). This is what the drive-reinforcement model predicts. As can be seen in Figure 11, the synaptic weight for $\mathrm{CS}_{1}$ remains unchanged during the 60 trials for which the computer simulation was run. Flaherty (1985) goes on to note that some conditioning has been reported for simultaneous CS and US onsets in the case of fear conditioning (Burkhardt \& Ayres, 1978; Mahoney \& Ayres, 1976). Thus, the experimental results with regard to simultaneous conditioning appear complex and it can only be noted that the predictions of the drivereinforcement model appear to be consistent with some of the experimental evidence.

For interstimulus intervals greater than zero, experimental results suggest that a nominal interval of $500 \mathrm{msec}$ (one time step in the simulations) is optimal when conditioning short-latency skeletal reactions. With longer intervals, the efficacy of conditioning declines until, for intervals exceed- ing a few seconds, no conditioning is observed (see review by Moore \& Gormezano, 1977). This is consistent with the predictions of the drive-reinforcement model. In Figure 11, it is seen that conditioning is most rapid for an interstimulus interval of one time step in the case of $\mathrm{CS}_{2}$, progressively slower for intervals of three and five time steps in the cases of $\mathrm{CS}_{3}$ and $\mathrm{CS}_{4}$, respectively, with no conditioning occurring for an interstimulus interval of six time steps in the case of $\mathrm{CS}_{5}$.

An alternative way of viewing the simulation results shown in Figure 11 is to see them as confirming the expected consequences of assigning the learning-rate constants, $c_{j}$, in the manner described earlier. Namely, $c_{0}$ and $c_{6}$ were set equal to zero, $c_{1}$ was assigned the highest value, and $c_{2}$ through $c_{5}$ were assigned progressively lower values. Thus, the simulation results in Figure 11 reflect the fact that the learning-rate constants were chosen consistent with the empirical evidence regarding interstimulus interval effects.

\section{Second-Order Conditioning}

Second-order conditioning is an experimental procedure in which one CS is reinforced by another CS, the latter CS having been reinforced previously by a US. Pavlov (1927/ 1960 ) reported that this procedure yielded conditioning in the second stage, the second CS coming to elicit the conditioned response originally elicited only by the first CS. However, in discussing second-order conditioning, Rescorla (1980) commented on "a historically nagging issue" and stated that the "issue concerns whether, in fact, secondorder conditioning is a real and powerful phenomenon. Although Pavlov reported its occurrence, he described it as transient. Subsequent authors have often been less than enthusiastic about its reality"' (pp. 3-4). This is interesting, because the drive-reinforcement model predicts that second-order conditioning will not be as strong as firstorder conditioning and that second-order conditioning will be transient. Simulation results that are the basis of this prediction are shown in Figure 12 where, in Stage 1 of conditioning, $\mathrm{CS}_{1}$ is reinforced by a US, achieving an asymptotic synaptic weight value of just a little more than 4 . After delay conditioning in Stage 1 (Trials 1-60), second-order conditioning occurs in Stage 2 (Trials 61-200). The drivereinforcement model predicts significantly weaker conditioning in Stage 2, the synaptic weight associated with $\mathrm{CS}_{2}$ peaking at a value between 1 and 2 . Furthermore, the transient nature of second-order conditioning, as reported by Pavlov (1927/1960), is predicted by the model. In Stage 2 of the simulated second-order conditioning experiment, after the $\mathrm{CS}_{2}$ synaptic weight peaks, the model predicts the subsequent decline of the weight due to what is essentially an extinction process. Had the simulation been carried out for further trials, the $\mathrm{CS}_{\mathbf{2}}$ synaptic weight would have declined asymptotically to the lower bound of 0.1 .

\section{Conditioned Inhibition}

Delay conditioning yields conditioned excitation; that is, the $\mathrm{CS}$ comes to excite the CR. An alternative procedure de- 
STIMULUS CONFIGURATION

AND RESPONSE:
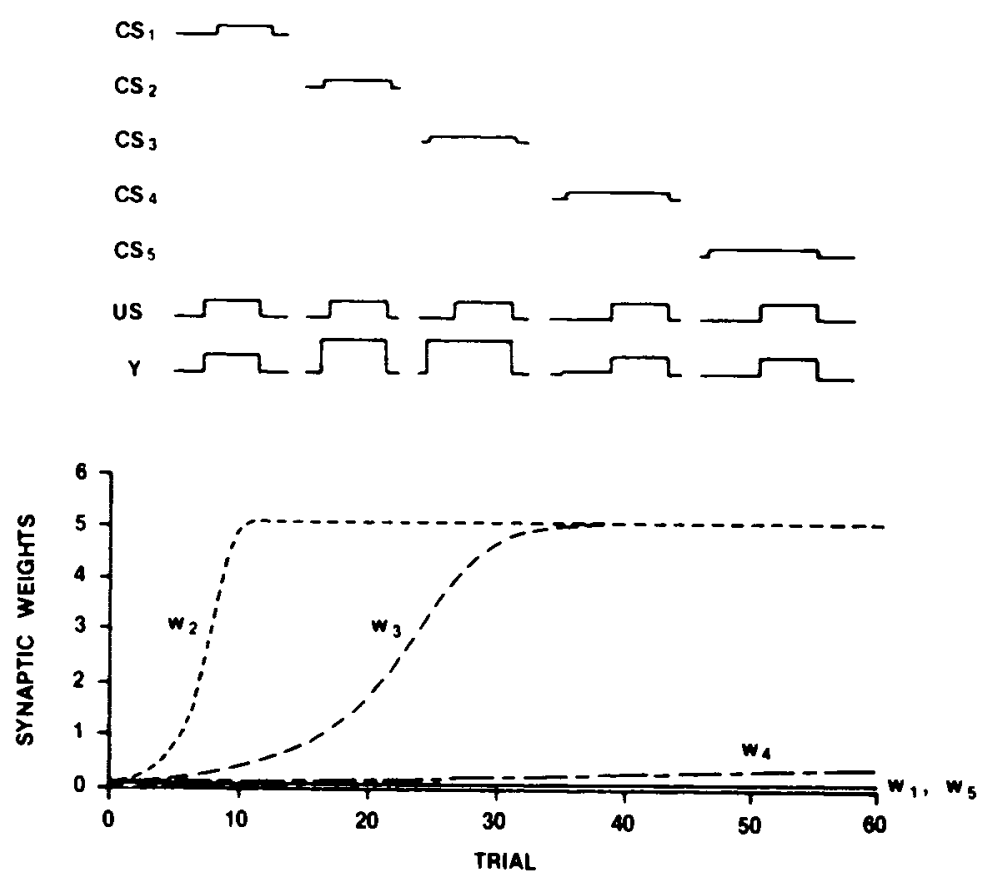

Figure 11. The drive-reinforcement model's predictions of the effects of the interstimulus interval. Consistent with the experimental evidence and consistent with the assignment of values to the learning-rate constants, $c_{\text {, }}$, the model is seen to predict no conditioning for simultaneous $\mathrm{CS}_{1}$ and US onsets and then decreased rates of conditioning as the interstimulus interval increases heyond the optimal interstimulus interval employed with $\mathrm{CS}_{2}$. Interstimulus intervals were as follows: zero time steps for $\mathrm{CS}_{1}$, one time step for $\mathrm{CS}_{2}$, three time steps for $\mathrm{CS}_{3}$, five time steps for $\mathrm{CS}_{4}$, and six time steps for $\mathrm{CS}_{6 .}$ (See text and Appendix for details.)

veloped by Pavlov (1927/1960) yields what he termed conditioned inhibition; that is, a CS would come to inhibit a CR that otherwise would have manifested.

In one of his procedures for demonstrating conditioned inhibition, Pavlov would utilize, in the first stage of conditioning, a delay conditioning procedure to render $\mathrm{CS}_{1} \mathrm{ex}$ citatory with respect to a CR. Then, in a second stage of conditioning, he would continue to reinforce $\mathrm{CS}_{1}$ with the US but would also present an unreinforced $\mathrm{CS}_{1}-\mathrm{CS}_{2}$ pair to the animal. During the second stage of conditioning, the animal's response to $C_{1}$ unpaired would decrease initially and then return to its original level. The animal's response to the $\mathrm{CS}_{1}-\mathrm{CS}_{\mathbf{2}}$ pair would decrease to zero. Furthermore, Pavlov was able to demonstrate that $\mathrm{CS}_{2}$ would become a conditioned inhibitor in that, after Stage 2 conditioning, if $\mathrm{CS}_{2}$ was paired with another $\mathrm{CS}$, say $\mathrm{CS}_{3}$, that was itself known to be a conditioned exciter, the $C R$ associated with $\mathrm{CS}_{3}$ was, in general, reduced or eliminated.

The drive-reinforcement model predicts this behavior, as can be seen in Figure 13. In Stage 1 (Trials 1-70) of the simulated conditioning, $\mathrm{CS}_{1}$ is reinforced by a US so that conditioned excitation develops, with the progress of the excitatory weight, $w_{1}(E)$, exhibiting the usual $S$-shaped acquisition curve. Then, in Stage 2 (Trials 71-200), $\mathrm{CS}_{1}$ un- paired is reinforced by the US once in each trial, while the $\mathrm{CS}_{1}-\mathrm{CS}_{2}$ pair is also presented once during each trial and the pair is unreinforced. The model predicts that the excitatory weight associated with $\mathrm{CS}_{1}$ will decrease initially and then return to its previous level, mirroring the behavior Pavlov observed with his animals. Also, the model predicts that the inhibitory weight, $w_{2}(\mathrm{I})$, associated with $\mathrm{CS}_{2}$ will grow stronger as Stage 2 conditioning proceeds, consistent with Pavlov's observation that $\mathrm{CS}_{\mathbf{2}}$ becomes a conditioned inhibitor. (Regarding the notation employed here, an E or an I in parentheses following $w_{l}$ signifies an excitatory or inhibitory weight, respectively. This notation involves a degree of redundancy inasmuch as excitatory weights will always be positive and inhibitory weights will always be negative, and, in the graphs, excitatory and inhibitory weights for a particular CS could be distinguished on that basis.)

Because the decrease in the excitatory weight associated with $\mathrm{CS}_{1}$ during the second stage of conditioning and then its subsequent return to the asymptotic level achieved in the first stage of conditioning may seem surprising, a few words of explanation may be in order. The initial decrease is due to the occurrence of the unreinforced $\mathrm{CS}_{\mathbf{1}}-\mathrm{CS}_{2}$ pair in that the onset of the $C S_{1}-C_{2}$ pair yields a positive $\Delta x_{1}$ that 
STIMULUS CONFIGURATION AND RESPONSE:

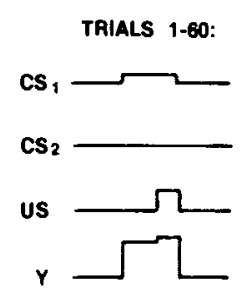

$$
\underset{\text { EXCITATION }}{\text { CONDITIONED }} \rightarrow \mid \leftarrow \begin{gathered}
\text { SECOND-ORDER } \\
\text { CONDITIONING }
\end{gathered}
$$

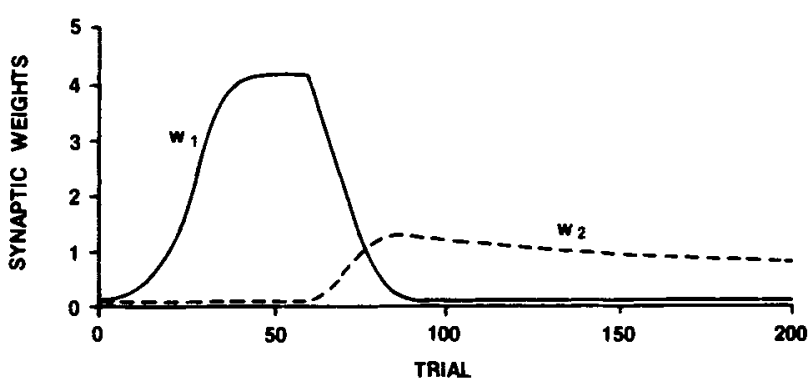

Figure 12. The drive-reinforcement model's predictions of the effects of second-order conditioning. Consistent with the experimental evidence, after delay conditioning in Stage 1 (Trials 1-60), the excitatory symaptic weight ascociated with $\mathrm{CS}_{1}$ extinguishes in Stage 2 (Trials 61-200) during second-order conditioning. Also consistent with the experimental evidence, the excitatory synaptic weight associated with $\mathrm{CS}_{2}$ increases initially during Stage 2 and then decreases. (See text and Appendix for details.)

is followed by a negative $\Delta y$ when $\mathrm{CS}_{1}$ and $\mathrm{CS}_{2}$ terminate. The negative $\Delta y$ occurs because, with an unreinforced pair, no US onset occurs at the time of $\mathrm{CS}_{1}-\mathrm{CS}_{2}$ offset, and thus there is nothing to cause the neuronal response to be sustained. The drive-reinforcement learning mechanism yields negative $\Delta w s$ whenever a positive $\Delta x_{i}$ is followed within $\tau$ time steps by a negative $\Delta y$. Thus, the excitatory weight associated with $\mathrm{CS}_{1}$ decreases initially in Stage 2 of conditioning. Similarly, the inhibitory weight associated with $\mathrm{CS}_{2}$ is decreasing (i.e., becoming more negative or becoming stronger in terms of its absolute value), because $\mathrm{CS}_{2}$ onset yields a positive $\Delta x_{2}$ that is followed by a negative $\Delta y$ at the time of $\mathrm{CS}_{1}-\mathrm{CS}_{2}$ offset. The excitatory weight associated with $\mathrm{CS}_{1}$ ceases to decrease and starts to increase when the conditioned inhibition becomes sufficient, such that the positive $\Delta y$ following the onset of $C_{1}$ unpaired with $\mathrm{CS}_{2}$ is larger than the negative $\Delta y$ following the onset of $\mathrm{CS}_{1}-\mathrm{CS}_{2}$ paired. The inhibitory weight associated with $\mathrm{CS}_{2}$ continues to decrease (become more strongly inhibitory), because its onset, yielding a positive $\Delta x_{2}$, continues to be followed by a negative $\Delta y$ until the conditioned inhibition of $\mathrm{CS}_{\mathbf{2}}$ becomes sufficient to cancel the conditioned excitation of $\mathrm{CS}_{1}$, at which point the $\mathrm{CS}_{2}$ inhibitory weight, $w_{2}(\mathrm{I})$, reaches its asymptotic level. At the same time, the $\mathrm{CS}_{1}$ excitatory weight, $w_{1}(E)$, approaches its asymptotic level, equal to its prior asymptotic level, because when the $\mathrm{CS}_{2}$ conditioned inhibition cancels the $\mathrm{CS}_{1}$ conditioned excitation, the reinforcement of $\mathrm{CS}_{1}$ unpaired is the only event in each trial that yields a nonzero $\Delta y$ following a positive $\Delta x$. Thus, toward the end of Stage 2 conditioning, the situation, in terms of positive $\Delta x$ s followed by nonzero $\Delta y$, is similar to that in Stage 1.

\section{Extinction and Reacquisition Effects}

When conditioned excitation develops in conjunction with a CS, as it did for $C_{1}$ at the conclusion of Stage 1 (Trials 1-70) and Stage 2 (Trials 71-200) of the conditioning shown in Figure 13, then, as Pavlov (1927/1960) observed, if the CS continues to be presented in a third stage of conditioning, this time without reinforcement, the CR extinguishes; that is, with a sufficient number of unreinforced presentations of the CS, the CR decreases in magnitude and reaches zero. In addition, Pavlov inferred that conditioned inhibition developed during the extinction process, because he observed "spontaneous recovery" of the CR with time and also more rapid reacquisition of the CR if reinforced presentations of the CS were resumed. The predictions of the drive-reinforcement model are consistent with Pavlov's observations and inferences. Note that in Stage 3 (Trials 201-300) of conditioning in Figure 13, where $\mathrm{CS}_{1}$ is presented without reinforcement, the $\mathrm{CS}_{1}$ excitatory weight, $w_{1}(\mathrm{E})$, declines and the $C S_{1}$ inhibitory weight, $w_{1}(I)$, grows stronger, until they cancel one another, at which time the $C R$ will no longer appear.

Perhaps a few words are in order regarding the phenomenon of spontaneous recovery following extinction. Spontaneous recovery refers to the tendency of an extinguished conditioned response to return after the CS is not presented for some period of time. It seems that spontaneous recovery could be due to the state of the nervous system's changing sufficiently with time so that the conditioned inhibition that may develop during the process of extinction becomes less effective. (As noted above, Pavlov, 1927/1960, believed that conditioned inhibition developed during the process of extinction. However, Rescorla, 1969, has stated that "there is only meager evidence bearing on this question"' [p. 87].) If the hypothesized conditioned inhibition were to become less effective because a change in the state of the nervous sytem resulted in fewer of the conditioned inhibitory synapses' being active, then it would become easier for the conditioned response to manifest again. If this explanation of spontaneous recovery is correct, a neuronal model would not be expected to predict the phenomenon. A network model would be required to generate the prediction.

In the third stage of conditioning in Figure 13, the drivereinforcement model makes one further prediction that has not yet been discussed. In this simulation, it was not only $\mathrm{CS}_{1}$ that was presented unreinforced in Stage 3; the $\mathrm{CS}_{\mathbf{1}}-\mathrm{CS}_{\mathbf{2}}$ pair was also presented unreinforced. Pavlov $(1927 / 1960)$ observed that, under these circumstances, the conditioned excitation associated with $\mathrm{CS}_{1}$ extinguished but the conditioned inhibition associated with $\mathrm{CS}_{2}$ did not. This is predicted by the drive-reinforcement model. Note that in 
STIMULUS CONFIGURATION ANO RESPONSE:
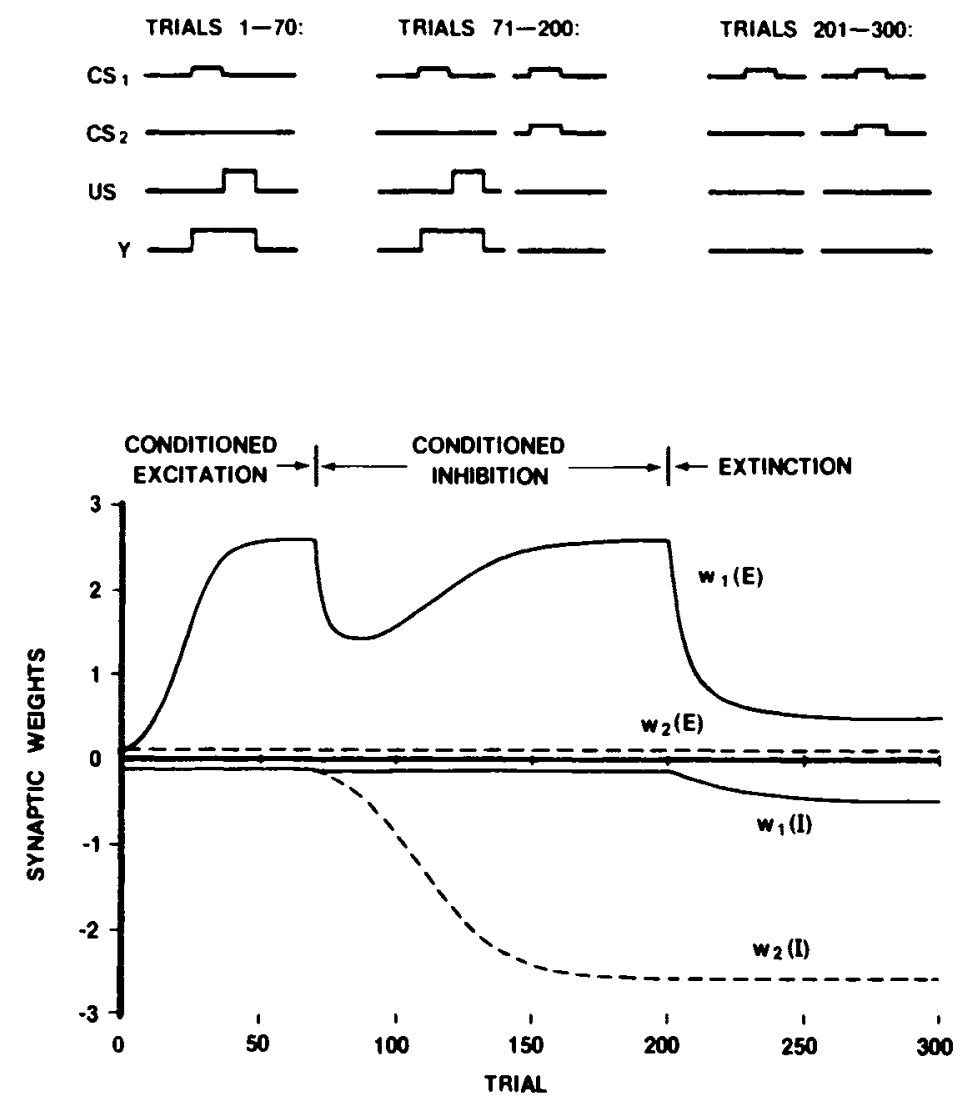

Figure 13. Results of a simulated classical conditioning experiment modeled after experiments performed by Pavlov (1927). In the simulated experiment, the drivereinforcement model's predictions are examined for three stages of conditioning. In Stage 1 (Trials 1-70), a delay conditioning paradigm is utilized and, consistent with the experimental evidence, an S-shaped acquisition curve for the excitatory synaptic weight, $w_{1}(E)$, ascociated with CS $_{1}$, is predicted. In Stage 2 (Trials $71-200$ ), Pavlov's conditioned inhibition paradigm is employed and time courses for the $\mathrm{CS}_{1}$ excitatory synaptic weight, $w_{1}(E)$, and the $C_{2}$ inhibitory synaptic weight, $w_{2}(I)$ are predicted consistent with Pavlov's observations that salivation to $\mathrm{CS}_{1}$ decreased initially and then returned to its previous asymptotic level and that $\mathrm{CS}_{2}$ became a conditioned inhibitor. In Stage 3 (Trials 201-300), an extinction paradigm is employed and, consistent with Pavlov's ohservations and inferences, the behavioral response to $\mathrm{CS}_{1}$ extinguishes [the sum of $w_{1}(E)$ and $w_{1}(I)$ approaches zero], the $\mathrm{CS}_{1}$ inhibitory synaptic weight, $w_{2}(I)$, undergoes a small increase in its absolute value, and the $\mathrm{CS}_{2}$ inhibitory synaptic weight, $w_{2}(I)$, does not extinguish. (See text and Appendix for details.)

the third stage of conditioning in Figure 13, the inhibitory weight, $w_{2}(\mathrm{I})$, remains unchanged during the unreinforced presentations of the $\mathrm{CS}_{1}-\mathrm{CS}_{2}$ pair. This prediction of the drive-reinforcement model differs from that of the Rescorla-Wagner model of classical conditioning. As Rescorla and Wagner (1972) pointed out, their model is inconsistent with the experimental evidence of conditioned inhibition studies in that the model predicts the extinction of conditioned inhibition. The drive-reinforcement model does not make this prediction, because the positive $\Delta x$ that occurs at the time of $\mathrm{CS}_{\mathbf{2}}$ onset is not followed by a positive $\Delta y$.
Pavlov (1927/1960) reported that if, after the extinction of a CR, reinforced presentations of the CS were resumed, then the $C R$ would be reacquired more rapidly than during the first series of reinforced trials. The drive-reinforcement model predicts this reacquisition effect, as can be seen in Figure 14, where delay conditioning occurs in Stage 1 (Trials 1-70), extinction of the CR occurs in Stage 2 (Trials 71-140), and reacquisition of the CR occurs in Stage 3 (Trial 141-200). When measured to an accuracy of three significant figures, the CS excitatory weight reached its asymptotic level in 61 trials in Stage 1 but reached the same level in only 47 trials in Stage 2 . This effect occurs because 
STIMULUS CONFIGURATION AND RESPONSE:

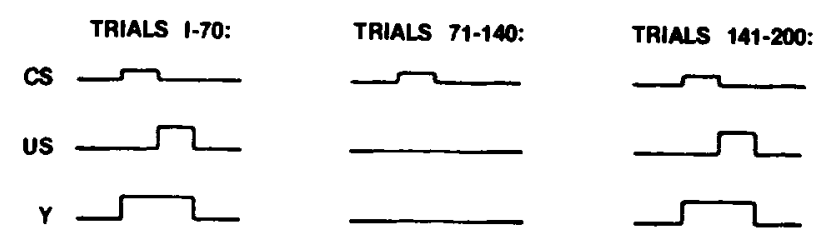

CONDITIONED
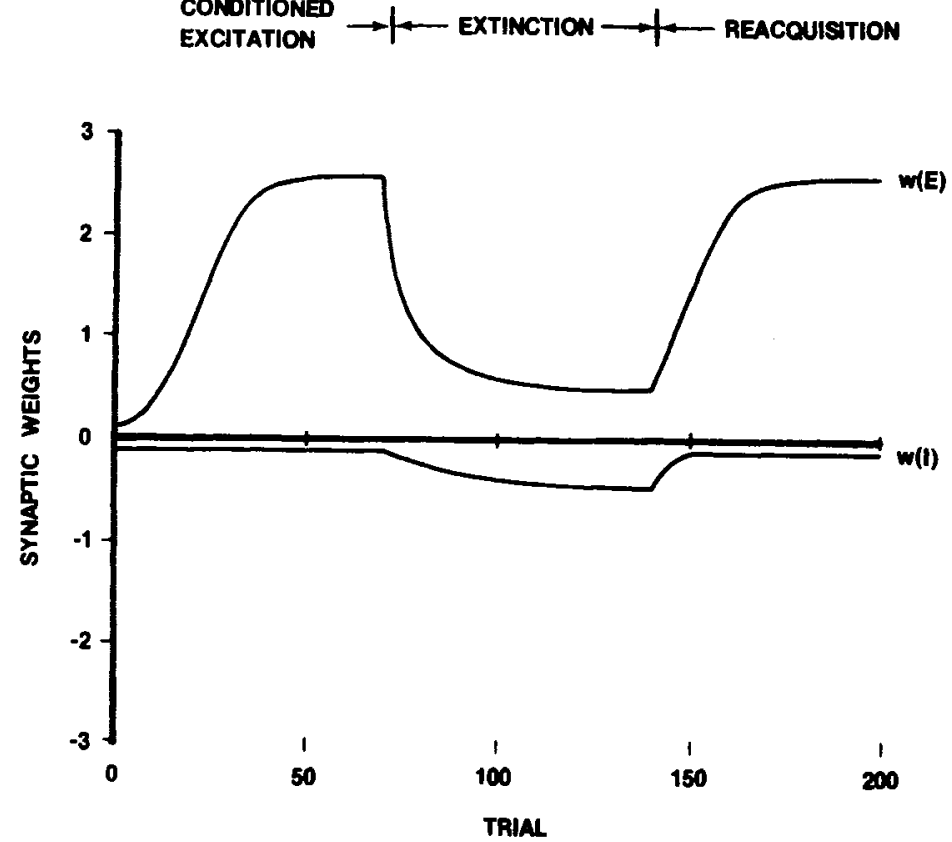

Figure 14. Results of a simulated three-stage classical conditioning experiment in which the drive-reinforcement model's predicted rate of reacquisition of a CR in Stage 3 (Trials 141-200) after extinction in Stage 2 (Trials 71-140) is compared with the predicted rate of initial acquisition in Stage 1 (Trials 1-70). Consistent with experimental evidence demonstrating that reacquisition occurs more rapidly, the drive-reinforcement model predicts that acquisition in Stage 1 will require 61 trials, compared with 47 trials for reacquisition in Stage 3. (See text and Appendix for details.)

the CS excitatory weight begins at a higher level during reacquisition than during the initial acquisition process. It may be noted that this prediction of the drive-reinforcement model differs from the predictions of the Rescorla-Wagner (1972) and Sutton-Barto (1981) models in that the latter two models do not predict the more rapid reacquisition of conditioned responses. Alternative explanations for reacquisition effects may be found in Kehoe (in press), in which a twolayer network model is proposed, and in Schmajuk and Moore (1987), in which a variant of the Moore-Stickney (1980) attentional model is considered.

\section{Backward Conditioning}

In backward conditioning, the onset of the US precedes the onset of the CS. There have been conflicting reports regarding whether backward conditioning leads to conditioned excitation or conditioned inhibition (see the review by Gormezano et al., 1983). Mahoney and Ayres (1976) sought to design experiments that would clarify some of the issues involved, and they concluded that conditioned excitation did result from backward conditioning. At this time, the consensus appears to be that backward conditioning can lead to conditioned excitation initially but that extended backward conditioning usually yields conditioned inhibition (Dolan, Shishimi, \& Wagner, 1985; Flaherty, 1985; Heth, 1976; Pavlov, 1928; Rescorla, 1969; Schwartz, 1984; Wagner \& Terry, 1975). The initial conditioned excitation may be due to transient effects associated with global brain processes, such as arousal triggered by the onset of the surprising US. In this view of backward conditioning, the hypothesized underlying process is one of conditioned inhibition that prevails with extended conditioning, after the US has come to be expected. The predictions of the drive-reinforcement model are consistent with this hypothe- 
(a)

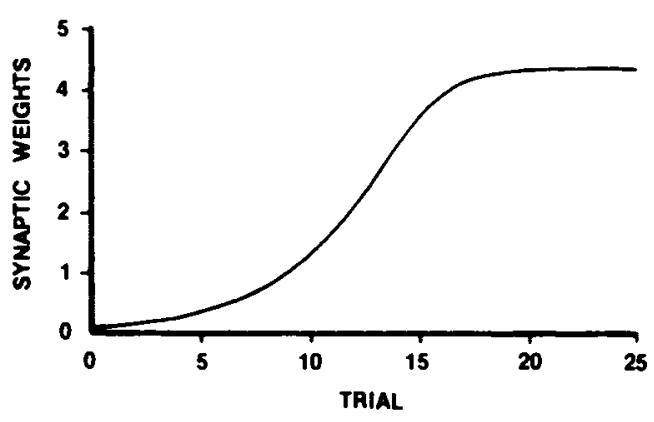

(b)

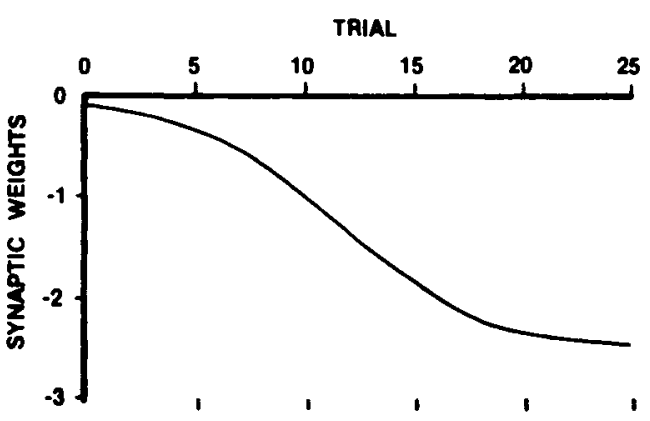

STIMULUS CONFIQURATION AND RESPONSE:

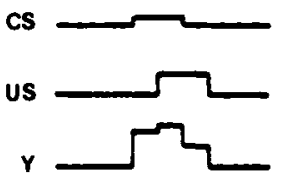

STIMULUS CONFIGURATION AND RESPONSE:

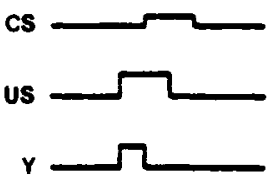

Figure 15. Results of simulated classical conditioning experiments in which the drivereinforcement model's predictions for (a) forward and (b) backward conditioning are contrasted. Consistent with the experimental evidence, the model predicts that conditioned inhibition will result from backward conditioning, in contrast to conditioned excitation being predicted as the result of forward conditioning. (See text and Appendix for details.)

sis, as can be seen in Figure 15. In Figure 15a, forward conditioning is shown for a CS, the onset of which occurs two time steps before the onset of the US. In Figure 15b, backward conditioning is shown for the same CS and US, in this case with the onset of the CS following the onset of the US by two time steps. The drive-reinforcement model predicts that backward conditioning will lead to conditioned inhibition, consistent with the experimental results obtained in most cases of extended backward conditioning. However, regarding these experimental results, J. W. Moore (personal communication, June 18, 1986) has suggested that one caveat is in order: "No studies have used the requisite combination of summation and retardation tests to assess the presumed learned inhibitory properties instilled by backward training."

\section{Blocking and Overshadowing}

Temporal contiguity between a CS and a US is fundamental to classical conditioning. This has long been understood. But although temporal contiguity is necessary, $\operatorname{Kamin}(1968,1969)$ has demonstrated that it is not sufficient. Kamin has shown that a CS must also have predictive value. That is to say, there must be a contingent relationship between the CS and US as well as a relationship of temporal contiguity; otherwise, no conditioning will occur. Kamin demonstrated this by first reinforcing $\mathrm{CS}_{1}$ with a US until an asymptotic level of associative strength was reached. Then he added $\mathrm{CS}_{\mathbf{2}}$ such that $\mathrm{CS}_{\mathbf{2}}$ was presented simultaneously with $\mathrm{CS}_{1}$ and both were reinforced. Kamin showed that no, or very little, associative strength developed between $\mathrm{CS}_{2}$ and the US. The first CS blocked conditioning of the second CS.

The drive-reinforcement model predicts the phenomenon of blocking, as can be seen in Figure 16. In this simulated blocking experiment, $\mathrm{CS}_{1}$ is reinforced by the US in the first stage of conditioning (Trials 1-100), until the $\mathrm{CS}_{1}$ excitatory weight has approached its asymptotic level. Then, in Stage 2 of conditioning (Trials 101-160), $C_{1}$ and $C_{2}$ are presented simultaneously and reinforced with the US. It is seen that the $\mathrm{CS}_{2}$ excitatory weight remains unchanged during the second stage of conditioning. Consistent with the experimental evidence, the drive-reinforcement model predicts that, due to the previous conditioning of $\mathrm{CS}_{1}$, conditioning of $\mathrm{CS}_{2}$ will be blocked by $\mathrm{CS}_{1}$.

US preexposure effects may be due to the phenomenon of blocking (Mis \& Moore, 1973). If an animal experiences a 
STIMULUS CONFIGURATION AND RESPONSE:

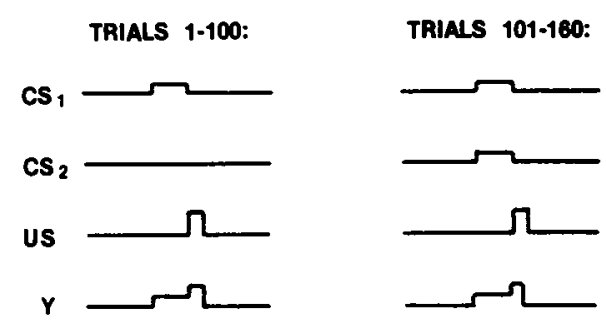

CONDITIONED EXCITATION $\rightarrow-$ BLOCKINO

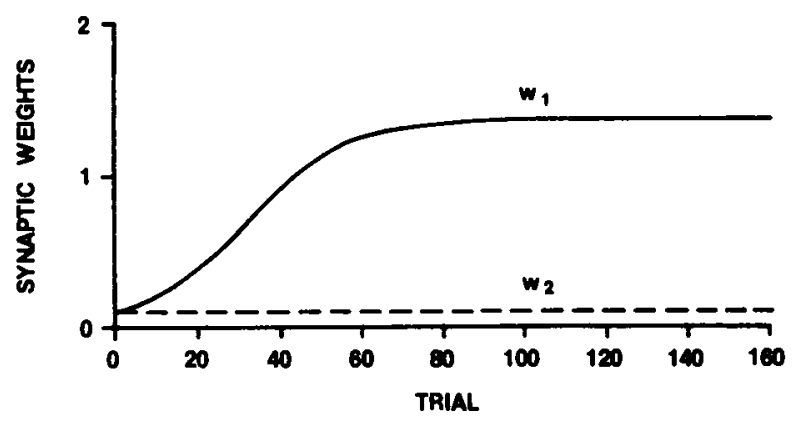

Figure 16. The drive-reinforcement model's predictions of the effects of a blocking stimulus. Consistent with the experimental evidence, the model predicts that after delay conditioning of $\mathrm{CS}_{1}$ in Stage 1 (Trials 1-100), conditioning of $\mathrm{CS}_{2}$, presented simultaneously with $\mathrm{CS}_{1}$ in Stage 2 (Trials 101-160), will be blocked. The $\mathrm{CS}_{\mathbf{2}}$ excitatory synaptic weight, $w_{2}$, does not change in Stage 2. (See text and Appendix for details.)

number of US presentations prior to experiencing paired presentations of a CS and the US, the result is that the conditioning process is retarded. This effect may be due to the experimental context, during US preexposure, becoming a blocker for subsequent conditioning (see, e.g., Balsam \& Schwartz, 1981, and a review by Flaherty, 1985).

A question in animal learning theory has been whether contingency aspects of classical conditioning derive from limitations on the amount of associative strength available, so that, in effect, stimuli must compete for the available associative strength (Rescorla \& Wagner, 1972), or whether, in effect, stimuli must compete for an animal's attention (Mackintosh, 1975; Moore \& Stickney, 1980, 1985; Sutherland \& Mackintosh, 1971). The alternative hypotheses are not mutually exclusive. The drive-reinforcement neuronal model's predictions are consistent with the hypothesis that there are limitations on the associative strength available to stimuli. However, the neuronal model does not rule out the involvement of higher level attention mechanisms.

In the case of the drive-reinforcement model, it can be seen that the limits on $\Delta y(t)$ serve to limit the amount of as- sociative strength available to competing stimuli. $y(t)$ is bounded such that it is less than or equal to $y^{\prime}(t)$, the maximal frequency of firing of the neuron. For nonoverlapping CSs and USs, the upper bound on $y(t)$ may actually be less than $y^{\prime}(t)$ because, in this case, $y(t)$ never exceeds the amplitude of the UR. Thus, as seen in Figure 16, if $\mathrm{CS}_{1}$ has been reinforced until an asymptotic level of conditioning is reached, subsequent conditioning of a second stimulus, $\mathrm{CS}_{2}$, will be blocked if the second stimulus forms a compound with the first and the onsets of $\mathrm{CS}_{1}$ and $\mathrm{CS}_{2}$ are simultaneous. What happens is that in Stage 1 of conditioning, the positive $\Delta x_{i}(t-j)$ associated with $\mathrm{CS}_{1}$ interacts with the subsequent positive $\Delta y(t)$ induced by the onset of the US, causing $C_{1}$ to grow and thus diminishing $\Delta y(t)$ with each trial. Eventually the positive $\Delta y(t)$ associated with US onset decreases to the point where its effect is cancelled by the subsequent effect of the negative $\Delta y$ associated with US offset. The amplitude of $C_{1}$ has grown to the point where there is no room for the generation of a net positive $\Delta y$ subsequent to a positive $\Delta x_{i}$ when $\mathrm{CS}_{2}$ is introduced as part of a compound. Thus, consistent with the experimental evidence and with the hypothesis of Rescorla and Wagner (1972) that there are limitations on the associative strength available to stimuli, the drive-reinforcement model predicts that conditioning will be blocked with respect to $\mathrm{CS}_{2}$.

A variant of blocking is overshadowing (see, e.g., Baker, 1968; Couvillon \& Bitterman, 1982), first reported by Pavlov (1927/1960), in which two or more simultaneous CSs are reinforced in a single stage of conditioning. In this type of experiment, it is observed that the more salient stimulus acquires the greatest associative strength, in effect, partially blocking conditioning of the other stimuli. The drivereinforcement model predicts overshadowing, as may be seen in Figure 17. In this simulated classical conditioning experiment, three simultaneous CSs are reinforced by a US. $C_{1}$ and $C S_{2}$ are of equal amplitude. The amplitude of $\mathrm{CS}_{3}$ is twice that of either of the other two CSs. Consistent with the experimental evidence, the drive-reinforcement model is seen topredict that the $\mathrm{CS}_{3}$ excitatory weight will achieve an asymptotic value that is substantially higher than the equal and lower asymptotic values achieved by the $\mathbf{C S}_{1}$ and $\mathrm{CS}_{\mathbf{2}}$ excitatory weights. This effect occurs with the drive-reinforcement model because the change in the presynaptic frequency of firing upon CS onset is greater for $\mathrm{CS}_{3}$ than for $\mathrm{CS}_{1}$ or $\mathrm{CS}_{2}$. Thus, the $\mathrm{CS}_{3}$ excitatory weight increases more rapidly, taking up a larger fraction of the total available associative strength than either the $\mathrm{CS}_{1}$ or the $\mathrm{CS}_{2}$ excitatory weight.

\section{Compound Conditioning}

In compound conditioning, multiple CSs are presented simultaneously or sequentially for reinforcement (or for nonreinforcement). Compound CSs have appeared in some of the simulated classical conditioning experiments discussed above, including those involving conditioned inhibition, blocking, and overshadowing.

A compound conditioning experiment reported by Rescorla and Wagner (1972) can be utilized as a test of the 
STIMULUS CONFIGURATION AND RESPONSE:
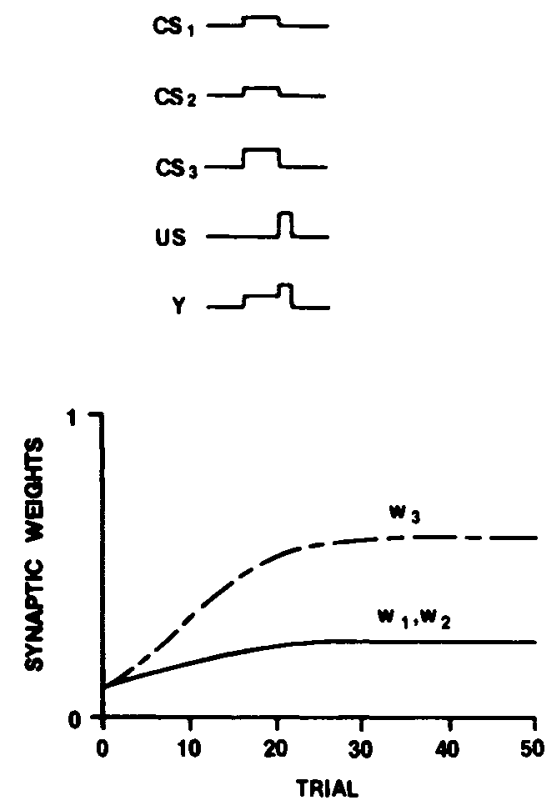

Figure 17. The drive-reinforcement model's predictions of the effects of stimulus salience on compound conditioning. Consistent with the experimental evidence, the model predicts that a more salient stimulus, $\mathrm{CS}_{3}$, which has an amplitude of $\mathbf{0 . 4}$, will condition more rapidly and strongly than less salient stimuli, $\mathrm{CS}_{1}$ and $\mathrm{CS}_{2}$, each with an amplitude of 0.2 . The asymptotic excitatory synaptic weight for $\mathrm{CS}_{3}$ is more than double that of either the $\mathrm{CS}_{1}$ or $\mathrm{CS}_{2}$ asymptotic excitatory synaptic weight. Thus, the drive-reinforcement model predicts the phenomenon of overshadowing. (See text and Appendix for details.)

drive-reinforcement model. The experimental results were obtained, Rescorla and Wagner noted, in a previously unpublished study by Wagner and Saavedra. The experiment involved comparing the effects of two CS-US configurations. In one configuration, $\mathrm{CS}_{1}$ occurring alone was reinforced and $C S_{1}$ paired with $C_{2}$ was also reinforced. An example of such a CS-US configuration appears in Figure 18a. In the second configuration, an example of which is shown in Figure $18 \mathrm{~b}, \mathrm{CS}_{1}$ occurring alone was not reinforced; only $\mathrm{CS}_{1}$ paired with $\mathrm{CS}_{\mathbf{2}}$ was reinforced. In the case of the first configuration, in which both $\mathrm{CS}_{\mathbf{1}}$ alone and $\mathrm{CS}_{\mathbf{1}}-\mathrm{CS}_{\mathbf{2}}$ paired were reinforced, the asymptotic associative strength of $\mathrm{CS}_{1}$ was observed to be high and that of $\mathrm{CS}_{2}$ was observed to be low. The ranking of the asymptotic associative strengths reversed when the second configuration was employed, in which $\mathrm{CS}_{1}$ alone was not reinforced and $\mathbf{C S}_{\mathbf{1}}-\mathrm{CS}_{2}$ paired was reinforced. These results are predicted by the drive-reinforcement model, as can be seen in Figure 18. In effect, the CS that more reliably predicts the US comes to block the other CS.

Space limitations preclude the presentation of additional results of computer simulations of compound conditioning experiments. However, two other compound conditioning effects that are predicted by the drive- reinforcement model should be noted. In the case of the overexpectation paradigm, two stimuli, $\mathrm{CS}_{1}$ and $\mathrm{CS}_{2}$, are first individually conditioned to an asymptotic level, each stimulus being reinforced with the same US. Then, in a second stage of conditioning, the two stimuli are presented as a compound that is reinforced using the same US as in the first stage. Rescorla and Wagner (1972) and Kremer (1978) reported that the associative strengths of the two stimuli decrease in the second stage of conditioning. Furthermore, if an initially neutral stimulus, $\mathbf{C S}_{3}$, is presented in compound with $\mathrm{CS}_{1}$ and $\mathrm{CS}_{2}$ in the second stage of conditioning, $\mathrm{CS}_{3}$ becomes a conditioned inhibitor. The drivereinforcement model predicts these effects.

In the case of superconditioning, the compound to be reinforced consists of two stimuli, one initially neutral and the other a conditioned inhibitor by virtue of prior conditioning. Reinforcement of this compound is observed to yield, for the initially neutral stimulus, an asymptotic associative strength that is greater than the corresponding associative strength in a control experiment in which both stimuli are initially neutral (Rescorla, 1971b; Wagner, 1971). The drive-reinforcement model predicts this effect.

\section{Discriminative Stimulus Effects}

The simulated classical conditioning experiments discussed above, the results of which were shown in Figure 18 , involved compound conditioning and discrimination learning. Discrimination learning experiments test an animal's ability to discriminate between reinforced and nonreinforced CSs. A more complex example of a compound conditioning experiment that tests for discriminative stimulus effects is shown in Figure 19a, where the compound $\mathrm{CS}_{1}-\mathrm{CS}_{3}$ is reinforced and the compound $\mathrm{CS}_{2}-\mathrm{CS}_{3}$ is not reinforced. For this CS-US configuration, experimental evidence reviewed by Rescorla and Wagner (1972) suggests that the asymptotic associative strengths will be high for $\mathrm{CS}_{1}$, low for $\mathrm{CS}_{3}$, and zero for $\mathrm{CS}_{2}$. Actually, $\mathrm{CS}_{2}$ is observed in the experiments to become a conditioned inhibitor. It can be seen in Figure 19a that the drive-reinforcement model predicts these results. Furthermore, the drive-reinforcement model predicts that the combined associative strengths of $\mathrm{CS}_{2}$ and $\mathrm{CS}_{3}$ will increase initially and then decrease. This transient effect, predicted by the model, has been observed by experimentalists, as Rescorla and Wagner (1972) noted.

A CS-US configuration similar to that shown in Figure 19a is shown in Figure 19b. Rescorla and Wagner (1972) reviewed the results of a study by Wagner, Logan, Haberlandt, and Price (1968) in which the discriminative stimulus effects of the CS-US configuration shown in Figure 19a were compared with the effects of the CS-US configuration shown in Figure 19b. The CS-US configuration shown in Figure $19 \mathrm{~b}$ represents a "pseudodiscrimination" experiment in that both compound CSs are reinforced sometimes and both are nonreinforced sometimes, so that it is actually a partial reinforcement experiment. Because of the similarity between the CS-US configurations in Panels $a$ and $b$ of Figure 19, 
(a)

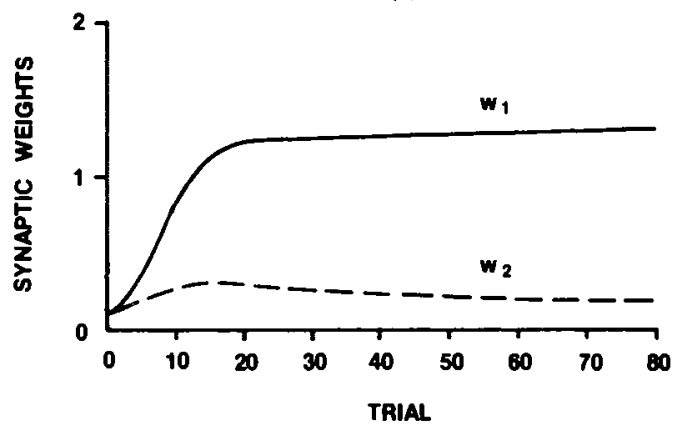

(b)

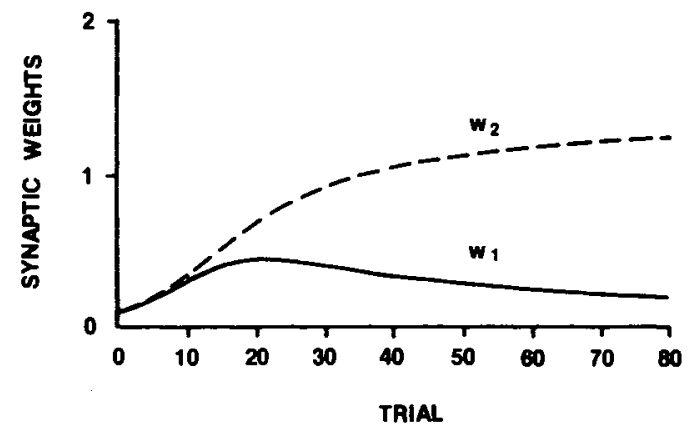

STIMULUS CONFIGURATION AND RESPONSE:

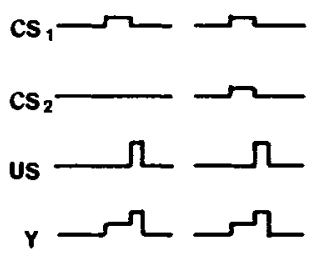

Figure 18. Results of simulated compound conditioning experiments in which the drive-reinforcement model's predictions for reinforced and nonreinforced CSs are compared. Consistent with the experimental evidence, in (a) the model predicts strong conditioning of $\mathrm{CS}_{1}$ relative to $\mathrm{CS}_{2}$, where both $\mathrm{CS}_{1}$ alone and the $\mathrm{CS}_{1}-\mathrm{CS}_{2}$ pair are reinforced. Again consistent with the experimental evidence, in (b) the model predicts that the ranking of asymptotic associative strengths for $\mathrm{CS}_{\mathbf{1}}$ and $\mathrm{CS}_{2}$ will be reversed with respect to (a) when $\mathrm{CS}_{1}$ alone is not reinforced and the $\mathrm{CS}_{1}-\mathrm{CS}_{2}$ pair is reinforced. (See text and Appendix for details.)

it is of interest to compare the experimental outcomes. Wagner et al. (1968) found that although $\mathrm{CS}_{3}$ was reinforced an equal fraction of the time in both discrimination and pseudodiscrimination training and occurred in compound with the same CSs, the eventual associative strength of $\mathrm{CS}_{3}$, when tested alone, was much greater after pseudodiscrimination training than after discrimination training. This is predicted by the drive-reinforcement model, as can be seen by comparing the asymptotic synaptic weights for $\mathrm{CS}_{3}$ in Panels a and $b$ of Figure 19. The net $\mathrm{CS}_{3}$ asymptotic synaptic weight (i.e., the $\mathrm{CS}_{3}$ asymptotic excitatory weight minus the absolute value of the $\mathrm{CS}_{3}$ asymptotic inhibitory weight) in Figure 19b is approximately double that of the net $\mathrm{CS}_{3}$ asymptotic synaptic weight in Figure 19a. It should be noted that the RescorlaWagner (1972) and Sutton-Barto (1981) models also correctly predict the experimental outcomes of the discrimination and pseudodiscrimination experiments just discussed, including the transient increase in the associative strength of the $\mathrm{CS}_{2}-\mathrm{CS}_{3}$ compound stimulus in the case of the discrimination training. However, as Wagner (1969) has noted, the Rescorla-Wagner model correctly predicts a higher asymptotic associative strength for $\mathrm{CS}_{3}$ in pseudodiscrimination training relative to discrimination training only when rate parameters in the model are constrained such that the rate parameter associated with reinforcement is greater than the rate parameter associated with nonreinforcement. Without requiring a comparable assumption, the drive-reinforcement model makes the same prediction.

\section{A Variant of the Drive-Reinforcement Neuronal Model}

The drive-reinforcement neuronal model, as specified above, requires that a positive change in presynaptic signal level occur for a synapse to be rendered eligible for a change in its efficacy. As noted earlier, the best argument for this constraint is that it yields a neuronal model that is consistent with the experimental evidence of classical conditioning. When the constraint is lifted so that $\Delta x_{i}(t-j)$ in Equation 2 is allowed to assume any value, positive or negative, the neuronal model then frequently generates predictions that deviate substantially from the experimental evidence. An example is shown in 
(a)

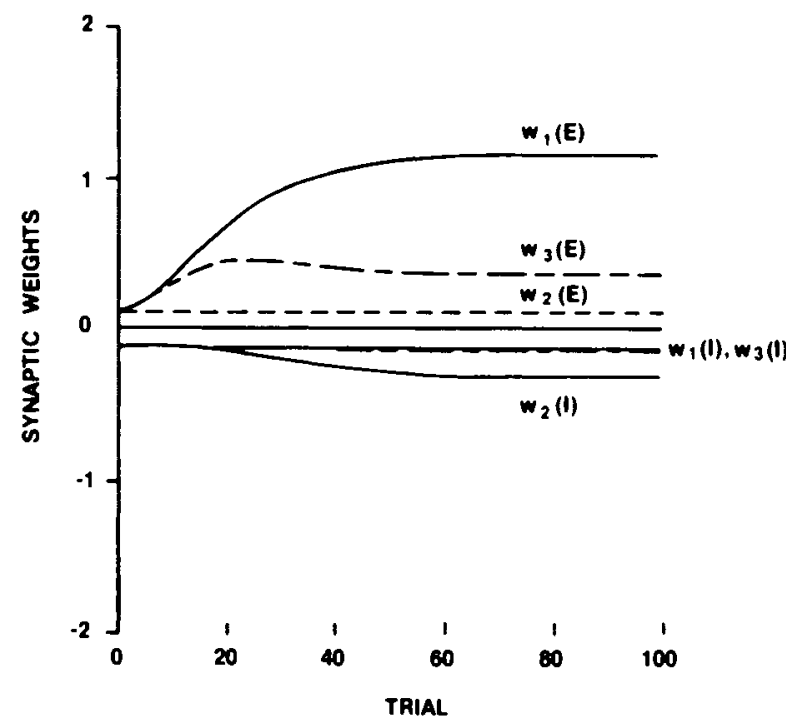

STIMULUS CONFIOURATION AND RESPONSE:

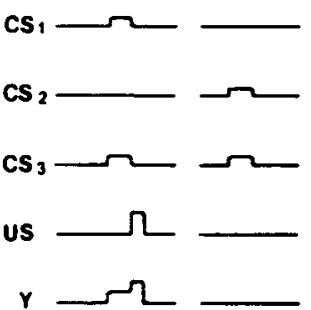

(b)

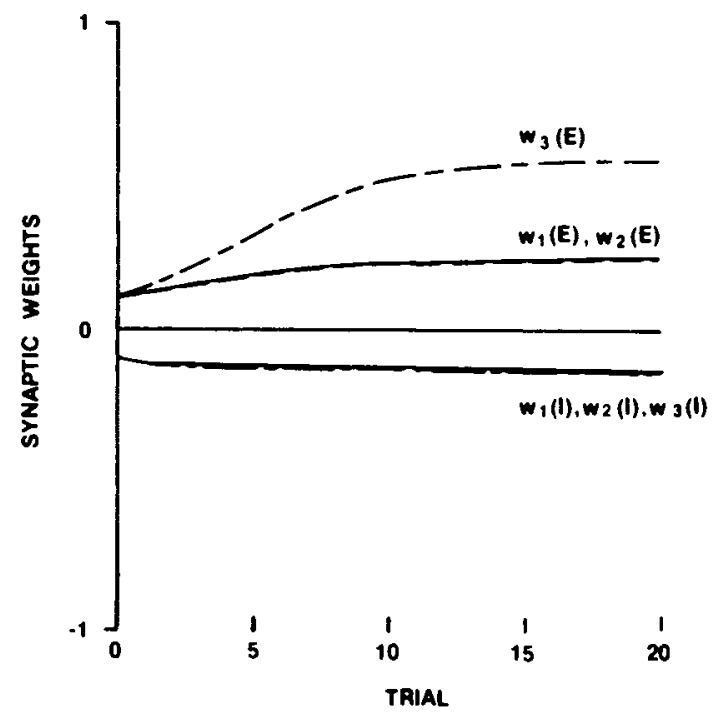

STIMULUS CONFIOURATION AND RESPONSE:

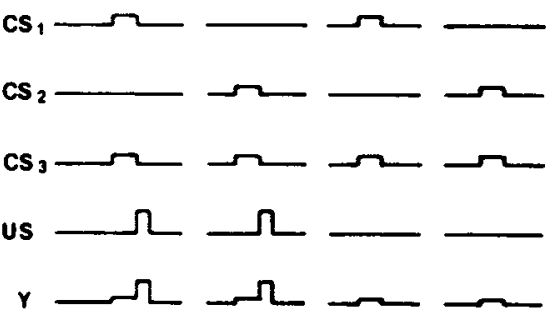

Figure 19. Results of simulated compound conditioning experiments in which the drive-reinforcement model's predictions of the effects of discriminative stimuli are determined for a more complex case than that portrayed in Figure 18. A discrimination learning paradigm is employed in (a). A pseudodiscrimination learning paradigm (actually, a partial reinforcement paradigm) is employed in (b). The model's predicted ranking of associative strengths in (a) and (b), as suggested by the asymptotic excitatory synaptic weights that are shown, is consistent with the ranking that is observed experimentally. Also, the predicted transient increase in the combined associative strengths of $\mathrm{CS}_{2}$ and $\mathrm{CS}_{3}$ in (a) has been observed by experimentalists. (See text and Appendix for details.)

Figure 20. The simulated classical conditioning experiment reported in Figure 20 is identical to one reported in Figure 16 (which was a blocking experiment), except that, in the case of Figure 20, $\Delta x_{i}(t-j)$ did not have to be greater than zero for the learning mechanism to be triggered. With this constraint removed, the $\mathrm{CS}_{1}$ and $\mathrm{CS}_{\mathbf{2}} \mathrm{ex}-$ citatory synaptic weights approach infinity, because the negative $\Delta x$ that occurs at the time of CS offset is multiplied by the negative $\Delta y$ that occurs at the time of US offset. The behavior that is plotted in Figure 16 is a clearcut prediction of the blocking phenomenon, whereas that plotted in Figure 20 bears no discernible relationship to experimentally observed behavior in the case of blocking experiments. 
STIMULUS CONFIGURATION AND RESPONSE:

TRIALS 1-100:

TRIALS 101-160:

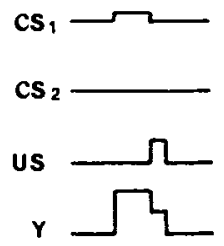

CONDITIONED EXCITATION $\rightarrow-\infty$ BLOCKING

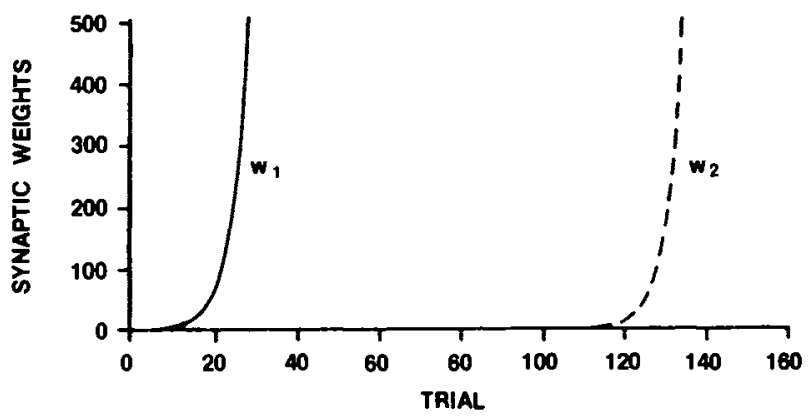

Figure 20. Results of a simulated blocking experiment that is identical to that reported in Figure 16, except that the drivereinforcement model utilized to generate the predictions in Figure 16 rendered a synapse eligible for a change in its efficacy, $w_{i}$, only upon the occurrence of a positive change in the presynaptic signal level. To generate the predictions shown here, a variant of the drivereinforcement model was employed, such that both positive and negative changes in presynaptic signal levels rendered a synapse eligible for a change in its efficacy. It is seen that the variant of the model employed here yields predictions that deviate markedly from the experimental evidence. Because these deviations are typical of this variant of the drive-reinforcement model, the other variant of the model, utilized to generate the predictions shown in Figures 4 through 19 , seems more likely to reflect the function of biological neurons. (See text and Appendix for details.)

\section{Summary}

By means of computer simulations of the drivereinforcement neuronal model, it has been shown that the model correctly predicts classical conditioning phenomena in the following categories: delay and trace conditioning, conditioned and unconditioned stimulus duration and amplitude effects, partial reinforcement effects, interstimulus interval effects, second-order conditioning, conditioned inhibition, extinction, reacquisition effects, backward conditioning, blocking, overshadowing, compound conditioning, and discriminative stimulus effects.

\section{DRIVES AND REINFORCERS}

The behavior of the proposed neuronal model may be understood in terms of two processes that involve postulated neuronal drives and reinforcers. If weighted presynaptic signal levels are defined to be neuronal drives and weighted changes in presynaptic signal levels are defined to be neuronal reinforcers, then the drive-reinforcement learning mechanism operates such that neuronal drive induction promotes learned excitatory processes and neuronal drive reduction promotes learned inhibitory processes. The interplay between these two processses yields the classical conditioning phenomena discussed above.

In this section, definitions of drives and reinforcers at the level of the single neuron and at the level of the whole animal will be examined further. Then the relationship of the drive-reinforcement neuronal model to animal learning theory will be discussed. I will begin by offering precise definitions of drives and reinforcers, definitions motivated by the neuronal model as it may be viewed in the context of animal learning theory.

\section{Definitions}

For the drive-reinforcement neuronal model, neuronal drives are defined to be the weighted presynaptic signals, $w_{i}(t) x_{i}(t)$. These weighted presynaptic signals drive the neuron. Equation 1 is termed the drive equation because it specifies how neuronal drives, $w_{i}(t) x_{i}(t)$, are transformed into neuronal behavior, $y(t)$. Neuronal reinforcers are defined to be the weighted changes in presynaptic signal levels, $w_{i}(t) \Delta x_{i}(t)$. Neuronal reinforcement results from the net effect of all of the weighted $\Delta x_{i}$ s experienced by a neuron at time $t$. Neuronal reinforcers thus are manifested as $\Delta y(t)$, and neuronal reinforcement is defined to be equal to $\Delta y(t)$. Note the distinction here: $A$ neuronal reinforcer is a weighted change in signal level that the neuron experiences at a single synapse; neuronal reinforcement is defined to be the collective effect of the neuronal reinforcers, manifested by the change in output, $\Delta y(t)$. Incremental neuronal reinforcement is defined to be an increase in the postsynaptic frequency of firing, and decremental neuronal reinforcement is defined to be a decrease in the postsynaptic frequency of firing, with both increases and decreases in firing frequency measured over intervals not exceeding a few seconds.

In the drive-reinforcement neuronal model, changes in presynaptic signal levels play two roles. A change in presynaptic signal level, $\Delta x_{i}(t)$, renders the $i$ th synapse eligible for future reinforcement. The synaptic weight, $w_{i}(t)$, for such an eligible synapse changes if a subsequent change in postsynaptic signal level, $\Delta y$, occurs not more than $\tau$ time steps in the future. The other role for $\Delta x_{i}(t)$, when weighted by $w_{i}(t)$, is to contribute to (i.e., partially or wholly cause) $\Delta y(t)$ and thus reinforce synapses rendered eligible by earlier changes in presynaptic signal levels. In effect, $\Delta x_{i}(t)$ looks to the future with regard to its role in rendering a synapse eligible for reinforcement and looks to the past in contributing to the reinforcement of synapses rendered eligible earlier. Equation 2, the neuronal learning mechanism, is termed the reinforcement equation because it specifies how neuronal reinforcers are transformed into changes in behavior. Thus, we see that Equation 1, the drive equation, involves the processing of signal levels to yield behavior, and Equa- 
tion 2, the reinforcement equation, involves the processing of changes in signal levels to yield learning.

It was noted earlier that the drive-reinforcement learning mechanism moves the onsets and offsets of pulse trains to earlier points in time. It should also be noted that, in doing this, the learning mechanism sets up the possibility of a chain of reinforcing events. Because of the way $\Delta x s$ and $\Delta y s$ interact in the model to yield $\Delta w s, \Delta y s$ come to occur earlier in time, making them available to reinforce even earlier $\Delta x \mathrm{~s}$. Thus, chains of reinforcing $\Delta x \mathrm{~s}$ and $\Delta y s$ can be established beginning with a primary reinforcer (which will be defined below).

Although the drive-reinforcement neuronal model appears complex relative to, say, the Hebbian model, this seems appropriate because the single neuron is coming to be recognized as a highly sophisticated cell. None of the operations proposed here seem incompatible with the known capabilities of the single neuron (see, e.g., Woody, 1982,1986 ).

The terms I have defined at a neuronal level mirror terms animal learning researchers have defined at the level of the whole animal. Additional terms may be defined in this way. For example, innate, or primary, neuronal drives may be distinguished from acquired neuronal drives. Primary neuronal drives are defined to have fixed synaptic weights. Acquired neuronal drives are defined to have variable synaptic weights, under the control of the neuronal learning mechanism. Primary neuronal drives will include deficit-related signals that have an internal source (drives to eat and drink are examples) and unconditioned stimuli that have an external source (food and water are examples). Acquired neuronal drives, likewise, are expected to have internal sources (as the result of possible conditioning, for example, of the hypothalamic reward and punishment centers) and to have external sources in the case of what becomes conditioned stimuli. The notion of acquired drives was first suggested by Miller (1951).

Psychologists have generally defined drives to include only the category of deficit-related internal signals. I am broadening the definition to include any signal that drives a neuron. My definition of primary drives comes closer to the conventional definition of drives, but, in this case, I still include (external) unconditioned stimuli as well as (internal) deficit-related signals. My point in changing the definition is to suggest that drives, defined in this broader fashion and at a neuronal level, can serve as the basis for a simpler and more rigorous learning theory.

I have noted that neuronal drives can have internal and external sources and can be primary (innate) or acquired. The same is true of neuronal reinforcers, as they have been defined above. Unconditioned stimuli, for example, function as primary drives, yielding unconditioned responses. Unconditioned stimuli also function as primary reinforcers, yielding conditioned responses. The drivereinforcement model suggests that when an unconditioned stimulus is functioning as a neuronal drive, it is the signal level, itself, that is important (see Equation 1), and when an unconditioned stimulus is functioning as a neuronal reinforcer, it is the onset and offset of the signal that is important (see Equation 2).

I have defined drives and reinforcers in a straightforward fashion at a neuronal level. However, such clearcut definitions have not proved to be possible at the level of the whole animal. For example, Toates (1985) remarked that the notion of drive "has been around for a long time. No one seems to know quite why we need the concept, but we keep putting it on display. It tends therefore to assume a variety of uncertain functions"' (p. 963). I am going to argue that we should not be surprised by this state of affairs. In the history of animal learning research, it has not been unusual for the notions of drives and reinforcers to be seen as problematic. When such notions are invoked at the level of the whole animal, this may be understandable. If the notions of drive and reinforcement are relatively straightforward at the level of the single neuron, as I am suggesting here, then we should not necessarily expect such notions to also be straightforward at higher levels. If neurons are classically conditionable cells in their own right, as the drivereinforcement model suggests, then, when millions or billions of such neurons interact in phylogenetically advanced nervous systems, the interactions may not be simple. The fact that we can make as much sense out of whole-brain function as we have, thanks to the dedication of animal learning researchers and many others, might even be seen as surprising, considering the complexity of the neural network of, say, a dog. The fact that Pavlov (1927/1960) and those who worked with and after him were able to see their way through to a relatively clear view of classical conditioning suggests that brain function may not be as complex as we might have expected. However, as Gray (1975) demonstrated in an especially careful and incisive analysis, complications arise with the notions of drives and reinforcers at the level of the whole animal.

If the notion of drive has been problematic at the level of the whole animal, what about the notions of drive reduction and drive induction, postulated to function as reinforcers (see, e.g., Mowrer, 1960/1973)? I have suggested that, at a neuronal level, drive reduction and induction have straightforward roles to play with respect to the process of reinforcement. Assuming, for the moment, that the hypothesized drive-reinforcement neuronal model is correct, how might we expect neuronal drive reduction and induction to map onto the level of the whole animal? Let us consider an example. The global reward, or "pleasure," centers discovered by Olds and Milner (1954) are known to be inhibitory (Fuxe, 1965), so they would be expected to yield decremental neuronal reinforcement. However, we know that the salivary reflex is excited by the taste of food. Also, the brain's global reward centers are presumably excited by the taste of food, 
but they will, in turn, deliver inhibition throughout the nervous system. This inhibition, in some cases, is likely to reach inhibitory interneurons and, thus, in effect, could be translated into excitation. Disinhibition is known to play a major role in the nervous system (Roberts, 1980). We can see, then, that there will be no clear-cut, simple mapping of excitation and inhibition into drives. Neither should we expect increases and decreases in excitation and inhibition (neuronal drive reduction and induction) to map in a clear-cut, simple way into global reinforcement (i.e., reward and punishment). In each case, the involved neural network will have to be considered before any mapping of neuronal drives and reinforcers into global drives and reinforcers can be established.

Evidence for this kind of complexity has been obtained by Keene (1973). Olds (1977) summarized Keene's findings as follows:

\begin{abstract}
A family of neurons excited by aversive brain shocks and inhibited by rewarding ones was identified in the intralaminar system of the thalamus; and a second family accelerated by rewards and decelerated by punishments was observed with probes in the preoptic area. (p. 95)
\end{abstract}

Keene's results demonstrate that the brain's global processes of reward and punishment can have opposite effects in different parts of the nervous system. Thus, the practical complexity of this situation at the level of the whole animal, reflecting perhaps the pragmatic decisions of the evolutionary process, may account for the problematic history of the psychological notions of drives and reinforcers.

\section{Relationship of the Drive-Reinforcement Neuronal Model to Animal Learning Theory}

Having defined drives, reinforcers, and related terms at a neuronal level, and having acknowledged the complexities that arise around these concepts at the level of the whole animal, I will now discuss how the drivereinforcement neuronal model relates to theories of animal learning.

In this century, the study of learning began with stimulus-response (S-R) association psychology (Guthrie, 1935; Pavlov, 1927/1960; Thorndike, 1911). In place of $S-R$ association psychology, the drive-reinforcement neuronal model suggests what could be called $\Delta S-\Delta R$ association psychology. The neuronal model suggests that it is not stimuli and responses that are associated but, rather, changes in stimuli and changes in responses, except, of course, in the theoretical model I am proposing, it is neuronal $\Delta S s$ and $\Delta R s$ that are associated and not the $\Delta S s$ and $\Delta R s$ of the whole animal. At the level of the whole animal, we can expect a more complicated situation, as I have already discussed.

Hull (1943) confronted the complexities that arise at the level of the whole animal. As Hilgard and Bower (1975) noted, Hull, in his herculean effort to systematize learning theory, was moving psychology from an S-R formulation to an $\mathrm{S}-\mathrm{O}-\mathrm{R}$ formulation, where $\mathrm{O}$ represented the state of the organism. Central to Hull's (1943) theory of learning was the definition of reinforcement as drive reduction. Hull (1952) went on to revise his position, concluding that reinforcement should be defined as drivestimulus reduction. Actually, Hilgard and Bower (1975) observed that "while favoring drive-stimulus reduction, Hull left the matter somewhat open, having vacillated between drive reduction and drive-stimulus reduction as essential to reinforcement"' (Hull, 1952, p. 153) (p. 167). The drive-reinforcement neuronal model suggests that Hull may have been right on both counts; both drive reduction and drive-stimulus reduction may function as reinforcers, because both can result in $\Delta y s$. Thus, at a neuronal level, the distinction between drive reduction and drive-stimulus reduction appears to dissolve. We see a reason why drives should probably be defined more broadly than Hull considered.

Hull's narrower definition of drives resulted in another problem for his theory. Hull's identification of drives and drive reduction with physiological needs or tissue deficits did not seem to leave room for such phenomena as animal play and the learning that results. Mishkin and Petri (1984) pointed out that

shortly after Hull developed [his] ideas, a number of studies on curiosity, manipulation and exploration suggested that other motives, not obviously related to physiological needs, also generated learning.... The recognition that there are motives that have no apparent basis in tissue deficits or other physiological needs was one major factor that eventually led to the demise of the drive reduction theory of learning

(Bolles, 1967). (p. 292)

The drive-reinforcement neuronal model solves the problems encountered with Hull's theories by moving from the level of the whole animal to the level of the single neuron, by suggesting a broader definition of drives, by allowing both drive reduction and drive induction to be reinforcing (consistent with Mowrer, 1960/1973), and by not necessarily identifying drive reduction with reward.

Regarding the relationship of drive reduction to reward, Gray (1975) discusses the question of whether rewards and punishments should be associated with drive decrements and increments, respectively. On the basis of Gray's analyses and those of others whom he cites, I have come to the conclusion that too close an identification of drive reduction with reward may not be warranted. The Darwinian process may have been more flexible in its approach as it evolved nervous systems. Therefore, I will not, in the theoretical framework I am proposing in this article, identify drive decrements with reward and drive increments with punishment even though, as generalizations, such identifications may be valid. There is nothing in the theoretical framework that requires such a rigid identification to make the theory workable.

After Hull, animal learning theory's next major step forward was due, in my opinion, to Mowrer (1960/1973). A colleague of Hull's at Yale, Mowrer moved from Hull's drive reduction (or drive-stimulus reduction) theory to a 
symmetric theory in which learning was attributed to both drive reduction and drive induction. Also, in Mowrer's theory, classical conditioning was accepted as basic. Mowrer's emphasis on classical conditioning and on symmetric processes in learning has had a strong influence on the theoretical framework I am proposing in this article.

Since Mowrer proposed his theory, substantial theoretical and experimental advances have been made in the understanding of classical conditioning phenomena (see, e.g., a review by Rescorla, 1988). Model systems, such as the rabbit nictitating membrane response, are providing a refined understanding of classical conditioning at psychological and neurobiological levels (see, e.g., Gormezano, 1972; Gormezano et al., 1983; Moore, 1979; Moore \& Gormezano, 1977; Thompson, 1976; Thompson, Berger, \& Madden, 1983). Also, the investigations of Kamin (1968) and Rescorla and Wagner (1972) have clearly demonstrated contingency aspects of classical conditioning as distinguished from contiguity aspects.

Along with an increased understanding of classical conditioning has come a growing conviction on the part of some animal learning theorists that classical conditioning phenomena are fundamental to animal learning; instrumental conditioning phenomena are then deemphasized by these theorists. Mowrer (1960/1973), early on, and Bindra $(1976,1978)$, more recently, have been leaders in this movement. The drive-reinforcement neuronal model is consistent with this view. If brains are, fundamentally, classically conditionable systems, then this may be because they are composed of classically conditionable neurons, as the drive-reinforcement model suggests. Instrumental conditioning phenomena are then seen to arise out of a neural substrate that utilizes classical conditioning mechanisms. As Bindra (1976) noted,

Once it is explicitly assumed that the production of any specific instrumental response or of some of its act components is linked to one or more particular eliciting stimulus configurations, then the way becomes clear for interpreting instrumental learning in terms of the learning of stimulus-stimulus contingencies alone. The problem of instrumental training then becomes one of making certain response-eliciting stimuli highly potent motivationally, and this can be done through stimulus-stimulus contingency learning between the response-eliciting stimulus and the incentive stimulus. (p. 245)

Research on autoshaping in which animals shape their behavior without a response-reinforcer contingency supports this position (Brown \& Jenkins, 1968; Jenkins \& Moore, 1973). As expressed by Flaherty, Hamilton, Gandelman, and Spear (1977), "the law of effect is apparently not necessary for the development of instrumental-like behavior" (p. 243).

Another way of viewing Bindra's theoretical position is as part of a movement away from drive reduction theories that emphasize internal deficit signals and toward incentive-motivation theories (Bindra, 1968; Bolles, 1972). Incentive-motivation theories suggest that "motivated behavior results not only from the push' of inter- nal, deficit signals but also from the pull' of external, incentive stimuli"' (Mogenson \& Phillips, 1976, p. 200; Mogenson \& Phillips's italics). It may be noted that neuronal drives, as defined earlier in this article, include both deficit signals, with their origin internal to the animal, and incentive stimuli, with their origin external to the animal.

While finding myself in sympathy with those who emphasize that classical conditioning is fundamental to learning, I do not want to go too far in that direction. Miller and Balaz (1981) note that classical conditioning has often been seen as involving the learning of stimulus-stimulus associations, while instrumental conditioning has often been seen as involving the learning of stimulus-response associations or, in the case of Mackintosh (1974), response-reinforcement associations. Frequently, animal learning theorists have chosen one particular class of associations as being fundamental and then have ruled out other classes of associations. Bindra (1976, 1978), for example, suggests that learning has to do not with the forming of stimulus-response associations, but rather with the learning of contingencies between stimuli. This question of which class of associations is fundamental to learning has been debated by animal learning theorists for decades. The drive-reinforcement neuronal model suggests that it may not be necessary to choose one class of associations over another. Solomon $(1981$, p. 2) observed: "One persisting question is 'what is learned?' The four candidates from the past were S-S associations, S-R associations, $\mathbf{R}$-reinforcer associations and $\mathbf{S}$-reinforcer associations.... It appears ... that associations of all four kinds can be established with the right procedures." The drive-reinforcement model allows for all four possibilities, suggesting that any of the four classes of associations will form when neuronal signals representing stimuli, responses, and reinforcers occur in appropriate temporal relationships. If a stimulus, response, or reinforcer results in a positive $\Delta x_{i}$ that is followed within the interval, $\tau$, by another stimulus, response, or reinforcer that yields a $\Delta y$ at the same neuron, then an association will form. Thus, an implication of the drive-reinforcement model is that, at a neuronal level, classical conditioning, instrumental conditioning, drive-reduction and induction, responsereinforcement, and incentive-motivation theories may all describe associations that can form in the nervous system. However, it is not the presence of signals representing stimuli, responses, or reinforcers that is required, according to the drive-reinforcement model, but rather changes in signal levels representing the onsets and offsets of stimuli, responses, and reinforcers.

\section{A Drive-Reinforcement Theory of Learning}

What kind of theory of learning is implied, then, by the drive-reinforcement neuronal model? At this point, I will sketch one possible form such a theory might take.

Three principles would appear to be fundamental to what I will call a drive-reinforcement theory of learning: (1) Primary neuronal drives are the foundation upon 
which all learning rests. (2) Neuronal reinforcers are changes in neuronal drive levels; neuronal drive induction promotes learned excitatory processes; neuronal drive reduction promotes learned inhibitory processes. Together, these processes yield acquired drives or learning. (3) The neuronal learning mechanism correlates earlier changes in presynaptic signals with later changes in postsynaptic signals, yielding changes in the efficacy of synapses. A change in the efficacy of a synapse is proportional to the current efficacy of the synapse.

If these principles should turn out to be correct at a neuronal level, how should we expect such mechanisms to be manifested at the level of the whole animal, or what I will call the network level? Neuronal drives might be expected to emerge at the network level as the positive and negative feedback loops that control behavior. As examples, consider a blood glucose detector that provides an internal primary-drive signal (this is what animal learning psychologists have customarily referred to as a drive) or the taste of food that provides an external primary-drive signal (what animal learning psychologists have customarily referred to as an unconditioned stimulus). These primary-drive signals are parts of innate negative feedback loops that are associated with what are termed the hunger drive and the salivation reflex. These feedback loops cause the blood glucose level to rise, because the animal is driven to each, and assist in causing food to disappear from the mouth and be digested, because the animal is driven to salivate. More generally, feedback loops representing drives include mating behavior, drinking behavior, behaviors associated with the approach to and consumption of prey, and behaviors associated with the attack of or flight from predators. In general, behaviors can be classified as approach or avoidance (Mowrer, 1960/1973). We might expect approach behavior to be supported by positive feedback loops and avoidance behavior to be supported by negative feedback loops. Positive and negative feedback loops that emerge at the level of the whole animal will be defined to be network drives, as distinguished from the neuronal drives defined earlier. Neuronal drives may be seen as the more atomistic basis of network drives. (See chapter 1 of Konorski, 1967, for a detailed discussion of what I am calling network drives.)

Primary network drives are the innate goals of the organism. Acquired network drives are the learned goals of the organism. On the basis of the hypothesized drivereinforcement neuronal learning mechanism, it is expected that acquired network drives are, in effect, constructed on top of the primary network drives. When primary (and acquired) drive levels vary, these variations in drive levels constitute reinforcement, and this reinforcement will spawn new drives (acquired positive and negative feedback loops). In this way, the process of learning is suggested to be sustained, with drives being built on top of drives. (Actually, in some cases, the process will not involve the acquisition of new drives so much as it will the refinement of current drives.) When acquired network drives become sufficiently complex, cognitive phenomena may begin to emerge.

To support the process of drive acquisition or learning at a network level, global centers that can broadcast generalized "start" and "stop" signals may be helpful. Such signals could serve to introduce appropriate $\Delta y$ s in a network at crucial times, thus rendering the overall activity of the network coherent. Such may be the roles of the global reward and punishment centers discovered, respectively, by Olds and Milner (1954) and by Delgado, Roberts, and Miller (1954). Consistent with this idea, global reward centers appear to employ inhibitory neurotransmitters (Stein, Wise, \& Belluzzi, 1977), which may function as "stop" signals, and global punishment centers appear to employ excitatory neurotransmitters (Stein et al., 1977), which may function as "start" signals. That a reward center should generate "stop" signals might seem paradoxical with respect to some behaviors, but disinhibitory mechanisms that are prevalent in the nervous system (Roberts, 1980) may make such an approach workable by enabling releasing mechanisms to be implemented where necessary. It should also be noted that if reinforcers are changes in drive levels, then global drive and reinforcement centers can be one and the same. A center's output will constitute a drive, and a change in a center's output will constitute a reinforcer. Consistent with this theoretical possibility, drive and reinforcement centers in the limbic system and hypothalamus appear to be so close together (Olds, 1977) as to be, perhaps, identical.

\section{EXPERIMENTAL TESTS}

In the computer simulations reported above, the drivereinforcement neuronal model has been demonstrated to be consistent, in general, with the experimental evidence of classical conditioning. However, such a demonstration involves comparing theoretical predictions of a neuronal model with experimental evidence obtained from whole animals. To some extent, whole-animal data has to be problematic vis-à-vis the predictions of a neuronal model. The effects of multiple interacting neurons, the effects of the brain's many interacting subsystems, and, in general, the effects of the global architecture of the brain will, of course, influence whole animal data. All of these effects, collectively, I will refer to as network effects to distinguish them from neuronal (meaning single neuron) effects. Network effects will preclude rigorous experimental tests of any neuronal model in terms of whole animal data. Tests at a neurobiological level will be required. Fortunately, such experimental tests are becoming feasible and, indeed, results to date encourage the notion that classical conditioning phenomena may manifest at the level of the single neuron, as the drive-reinforcement model suggests. (See reviews by Byrne, 1987; Carew \& Sahley, 1986; Farley \& Alkon, 1985; Kandel \& Spencer, 1968; Mpitsos, Collins, \& McClellan, 1978; Thompson, Berger, \& Madden, 1983; Woody, 1986. See also Hawkins \& Kandel 1984; Kelso \& Brown, 1986.) Instrumental con- 
ditioning experiments at the level of the single neuron are also becoming feasible (Stein \& Belluzzi, 1988).

At this point, perhaps a note is in order regarding the terms I am adopting. When I suggest that a single neuron may manifest classical conditioning phenomena, the "single neuron" I am referring to includes the synapses that impinge upon it. Those synapses, of course, come from other neurons or from sensory receptors, and, in that sense, what I am referring to as a phenomenon involving a "single neuron" is, in fact, a multineuron or neuron and receptor phenomenon. The point, though, is that it is a single neuron that may be undergoing the conditioning, as distinguished from alternative theoretical models that can be envisioned, in which whole circuits consisting of many neurons would be the lowest level at which conditioning could occur. An implication of the drivereinforcement neuronal model is that classical conditioning is not an emergent phenomenon but, rather, that the ability to undergo classical conditioning is a fundamental property of single cells.

Actually, the hypothesized drive-reinforcement learning mechanism could be implemented at a lower level than that of the single neuron. Minimally, what would seem to be required would be two synapses interacting such that one synapse would deliver the signal corresponding to $\Delta x_{i}(t-j)$, reflecting the onset of the CS, and the other synapse would deliver the signal corresponding to $\Delta y(t)$, reflecting the onset or offset of the US. Evidence of such interactions between synapses has been obtained in investigations of classical conditioning in Aplysia. The learning mechanism appears to involve what is termed activitydependent amplification of presynaptic facilitation (Hawkins, Abrams, Carew, \& Kandel, 1983) or activitydependent neuromodulation (Walters \& Byme, 1983) of sensory neuron terminals. The optimal interstimulus interval between activation of the sensory neuron terminal representing the $\mathrm{CS}$ and activation of the facilitator neuron terminal representing the US has been found to be about 500 msec (Carew, Walters, \& Kandel, 1981; Hawkins, Carew, \& Kandel, 1986). Although the evidence for conditioning at a neuronal level in Aplysia has been interpreted as suggesting a presynaptic learning mechanism, Farley and Alkon (1985) indicate that the sites of the changes may not be exclusively presynaptic.

Whether presynaptic of postsynaptic processes, or both, underlie learning is a question that has been investigated theoretically (Zipser, 1986) and experimentally (Carew, Hawkins, Abrams, \& Kandel, 1984). In this article, I have formulated the drive-reinforcement learning mechanism in terms of postsynaptic processes, although, as discussed above, the learning mechanism could be implemented in an exclusively presynaptic form. Apart from activitydependent amplification of presynaptic facilitation or activity-dependent neuromodulation offering a possible implementation of the drive-reinforcement learning mechanism, other possibilities can be envisioned that would still involve less than a whole neuron. Portions of dendritic trees and their impinging synapses might func- tion in a manner analogeus to the model I have envisioned for the whole neuron. Thus, there is a range of possibilities for implementation of the drive-reinforcement learning mechanism, extending from what is perhaps a minimal two-synapse interaction on the low end, ranging through portions of dendritic trees functioning as a basic unit of learning, up through the level at which a single neuron functions as the basic unit, and beyond to the point where the whole organism is treated as a single unit. Variations of the drive-reinforcement model may have relevance at each of these levels, even though the learning mechanism seems to lend itself naturally to implementation at a neuronal level.

Regarding the question of how the drive-reinforcement model can be tested at a neuronal level, synaptic inputs will have to be controlled and monitored precisely for a single neuron while the neuron's frequency of firing is continually monitored. It will be necessary to measure the direction and preferably also the magnitude of the changes in efficacy of affected synapses. Changes in synaptic inputs, as potential CSs, and changes in neuronal outputs, representing potential reinforcement, will have to be tested to determine which, if any, input and output patterns yield changes in the efficacy of synapses. In this way, it can be established whether onsets and offsets of hypothesized neuronal CSs and USs determine the efficacy of synapses in the manner specified by the drive-reinforcement model.

Experimental evidence that bears on the question of neuronal learning mechanisms has been obtained from studies involving the phenomenon of long-term potentiation (LTP). The results have been interpreted to suggest that neurons are Hebbian in character with respect to their learning mechanisms (Kelso, Ganong, \& Brown, 1986; Levy, 1985; Levy \& Desmond, 1985). However, the relationship of the phenomenon of LTP to learning is unclear at this time (Morris \& Baker, 1984). As Bliss and Lomo (1973) pointed out in the article in which they reported their discovery of LTP, "whether or not the intact animal makes use in real life of a property which has been revealed by synchronous, repetitive volleys to a population of fibres the normal rate and pattern along which are unknown, is another matter" (p. 355).

Recent experimental results involving LTP suggest that sequential neuronal inputs may be more efficacious than simultaneous inputs in causing synaptic weight changes to occur. Larson and Lynch (1986) have shown that brief high-frequency pulse trains delivered to nonoverlapping sets of synapses of hippocampal neurons are most effective in inducing LTP if the pulse train to a first set of synapses precedes a pulse train to a second set by $200 \mathrm{msec}$. With this experimental procedure, LTP is induced only in the second set of synapses. LTP is not induced in either set of synapses if the delay is reduced to zero or extended to 2 sec.

Recently, long-term depression (LTD) of parallel fiber test responses after conjunctive stimulation of parallel and climbing fiber inputs has been demonstrated in the cerebellum (Ito, 1986; Ito, Sakurai, \& Tongroach, 1982). 
However, the relationship of this phenomenon to classical conditioning is unclear at this time because, as Byrne (1987) notes,

activation of parallel fiber input during the period between $20 \mathrm{~ms}$ prior and $150 \mathrm{~ms}$ after climbing fiber stimulation were roughly equivalent in inducing LTD [Ekerot \& Kano, cited in Ito, 1984]. This indicates that the neural analog of the US (climbing fiber input) can induce a change in the neural analog of the CS (parallel fiber input) even if the CS occurs after the US. Therefore the intrinsic mechanism could support backward conditioning, a phenomenon that is not observed with behavioral conditioning. (p. 411)

Additional experimental evidence relevant to the question of neuronal learning mechanisms has been obtained by Baranyi and Ferher $(1978,1981 a, 1981 b, 1981 c)$, who have attempted to classically condition pyramidal neurons in the cat's motor cortex. CSs in the form of presynaptic activity were paired with USs in the form of postsynaptic cell firing. Evidence of conditioning was obtained in the form of enhanced excitatory postsynaptic potentials, with the enhancement being sustained for up to $41 \mathrm{~min}$. The relationship of these experimental results to classical conditioning phenomena remains to be demonstrated, however, because evidence of conditioning was obtained for interstimulus intervals ranging from 0 to $400 \mathrm{msec}$ and for either forward or backward pairing of the CS and US.

In summary, Baudry (1987), in a group report from a Dahlem Workshop, offered this assessment of some of the experimental evidence discussed above:

For discrete stimulus-response learning (i.e., skeletal muscle responses), no learning occurs with backward (UCS first) or simultaneous onset or in fact until the CS precedes the UCS by nearly $100 \mathrm{~ms}$. Learning is best with intervals from 200 to $400 \mathrm{~ms}$ and decreases as the interval is lengthened further. In terms of current models, the Aplysia system seems to follow this function remarkably well and this seems also to be the case for Hermissenda [Lederhendler \& Alkon, 1986]. It is not yet clear how LTP and LTD could satisfy this function although the newly described paradigm to obtain LTP [Larson \& Lynch, 1986] also seems to follow this temporal specificity. (p. 168)

\section{DISCUSSION}

The learning mechanism underlying nervous system function (if, indeed, there is a single basic mechanism) may not be of the character suggested by the Hebbian neuronal model. The Hebbian model suggests that approximately simultaneous neuronal signals are associated. The drive-reinforcement neuronal model, on the other hand, suggests that sequential changes in neuronal signals are associated. An implication of the drive-reinforcement model is that nervous systems, in effect, pay attention to changes, encoding causal relationships between changes as the basis for learning.

Besides psychology and neuroscience, several other disciplines have been addressing questions related to learning. These disciplines include (1) the cybernetically oriented efforts referred to as connectionist or neural network modeling, (2) artificial intelligence research, and (3) adaptive control theory and adaptive signal processing. In this section, the implications of the drivereinforcement neuronal model for each of these approaches will be considered.

\section{Connectionist and Neural Network Modeling}

For a few decades now, neural network models, or what are sometimes more generally referred to as connectionist models, have been proposed as theoretical models of nervous system function. Connectionist models have also been proposed as engineering solutions to problems, without any claim of biological relevance. In either case, with or without the claim of biological relevance, the thrust of connectionist modeling has been to address the issues of memory, learning, and intelligence by means of cybernetically oriented designs for massively parallel systems (Barto, 1985; Feldman, 1985; Grossberg, 1982; Hinton \& Anderson, 1981; Klopf, 1982; Kohonen, 1984; Levine, 1983; McClelland \& Rumelhart, 1986; Rumelhart \& McClelland, 1986).

In recent years, several approaches to connectionist modeling that have come to the fore appear to have promise in terms of solving the problem of accomplishing learning in large, deep networks. The ultimate potential of these approaches cannot be assessed yet, because efforts to scale up the respective connectionist networks are only beginning. What can be done at this point, and what I will attempt to do here, is to assess some of the approaches for their relevance to animal learning.

One dimension along which connectionist models may be assessed has to do with the nature of the feedback the models require from their environments. Some connectionist models operate in a strictly open-loop fashion, requiring no feedback from their environment. An example is Fukushima's (1980; Fukushima \& Miyake, 1982) connectionist model. Fukushima's network, when presented with spatial patterns, adjusts connection weights so that the patterns tend to cluster in ways that are useful for some purposes of pattern classification. No feedback from the environment is given or required. One question that arises is whether networks that operate in this way, in an open-loop or nongoal-seeking fashion, can be relevant to biological information processing. An implication of the drive-reinforcement neuronal model and of the learning theory implied by the model is that feedback loops through the environment are a fundamental part of biological information processing. In biological systems, it appears that the positive and negative feedback loops that constitute network drives, support goal-seeking, and that the changes in the levels of activity of these closed loops, or network drives, constitute reinforcement. It would seem that learning mechanisms internalize experienced causal relationships. In not encoding causal relationships, clustering mechanisms appear to be fundamentally different.

Nearest neighbor classifications of spatial patterns, like that accomplished with Fukushima's clustering technique, can also be accomplished with Boltzmann machines (Ac- 
kley, Hinton, \& Sejnowski, 1985; Hinton \& Sejnowski, 1986; Hinton, Sejnowski, \& Ackley, 1984) and what are sometimes called Hopfield networks (Cohen \& Grossberg, 1983; Hopfield, 1982, 1984; Hopfield \& Tank, 1985, 1986; Tesauro, 1986). Having been inspired by theoretical models in physics, the latter two classes of connectionist models utilize symmetric connections and what may be called adaptive equilibrium processes, in which the networks settle into minimal energy states. The networks have been demonstrated to have interesting and potentially useful properties, including, for example, in the case of Hopfield networks, solving analogues of the traveling salesman problem. However, symmetric network connections and adaptive equilibrium processes have not yet been demonstrated to be relevant to the modeling of nervous system function, at least with regard to the underlying learning mechanisms. It may be noted that a wide range of classical conditioning phenomena are predicted by the drive-reinforcement neuronal model, and it uses no symmetric connections or adaptive equilibrium processes. What the drive-reinforcement neuronal model does utilize is the real-time operation of drives and reinforcers that can be understood in terms of a network's ongoing, closed-loop interactions with its environment.

Continuing to look at connectionist models in terms of the nature of the feedback they require from their environment, a class of models that might be considered to be the other extreme from open-loop models are those using supervised learning mechanisms. Such network models require detailed feedback in the form of an error signal that indicates the difference between the desired output and the network's actual output. Rosenblatt (1962), Widrow (1962), and subsequently many others have investigated connectionist models utilizing supervised learning mechanisms. For these network models, error signals play no role in a theoretical neuron's computations relative to its input-output relationship, their only role being to instruct the neuron with regard to the modification of its synaptic weights. Supervised learning mechanisms introduce the need for a "teacher"' to provide a learning system with desired responses. In contrast, the drivereinforcement neuronal model, like some other real-time learning mechanisms, does not require the introduction of a teacher and, thus, is an example of an unsupervised learning mechanism. In the case of the drivereinforcement neuronal model, fixed (nonplastic) synapses that mediate USs function like an internal teacher or goal specification.

It should be noted that unsupervised learning mechanisms have sometimes been associated with systems that operate in an open-loop mode with respect to their environment. Unsupervised learning mechanisms have also been associated with clustering techniques as an approach to pattern recognition. However, as defined here, unsupervised learning mechanisms represent the class of learning mechanisms that do not require a teacher and therefore may be utilized in learning systems that operate in either open- or closed-loop modes with respect to their environment.
The distinction between unsupervised learning mechanisms that do not require a teacher and supervised learning mechanisms that do require a teacher would appear to be of fundamental importance. Although supervised learning mechanisms may have a role to play in artificial intelligence, it would seem that only unsupervised learning mechanisms are likely to be relevant to the modeling of natural intelligence. In general, biological systems accomplish learning without a teacher's being present in any explicit sense. Of course, a biological system's environment always functions as a teacher in an implicit sense, but that is exactly what real-time unsupervised learning mechanisms can take into account, as could be seen in the results of the computer simulations of the drivereinforcement model presented above.

One qualification is in order regarding the role of supervised learning mechanisms in natural intelligence. It is clear that something like supervised learning mechanisms play a large part in natural intelligence at higher, cognitive levels. At such levels, explicit teachers play an important role. However, I suggest that this has misled neural network modelers, causing them to introduce supervised learning mechanisms at a fundamental level. It is this hypothesized fundamental role for supervised learning mechanisms that I think is unlikely to be valid in the case of neural network or connectionist models, if the models are intended to be relevant for natural intelligence.

Regarding connectionist models that employ supervised learning mechanisms, the most promising recent form of this class of models is that developed by Werbos (1974), Parker (1982, 1985), Le Cun (1985), and Rumelhart et al. $(1985,1986)$. They have proposed mechanisms for propagating error signals from the output layer back to the input layer of a network. The performance of the resulting networks has been encouraging, and, therefore, the question arises of whether these connectionist models may be relevant to understanding animal learning. Such relevance seems unlikely for two reasons, that, in part, I have already discussed. First, animals do not receive error signals during learning except, in the case of humans, after a fairly high level of cognitive function has been achieved. Second, the drive-reinforcement neuronal model demonstrates that, at least for classical conditioning phenomena that appear to be fundamental to learning, backpropagating error-correction mechanisms are not required.

Recognizing that animal learning does not, in general, involve evaluative feedback from the environment, some investigators have moved away from supervised learning in which error signals must be provided to the learning system. A step in the direction of unsupervised learning is reinforcement learning (Barto \& Anandan, 1985; Barto \& Anderson, 1985; Barto \& Sutton, 1981a, 1981b; Farley \& Clark, 1954; Minsky, 1954; Sutton, 1984), or what Widrow, Gupta, and Maitra (1973) have called learning with a critic. Williams $(1986,1987)$ noted that in this type of learning the network may be provided with performance feedback as simple as a scalar signal, termed 
reinforcement, that indicates the network's degree of success. Reinforcement learning networks have been demonstrated to be workable (e.g., see Barto et al., 1983), at least in the case of small-scale versions. Furthermore, reinforcement learning networks appear more likely to be biologically relevant than supervised learning networks, because less evaluative feedback is required from the environment. However, an implication of the drivereinforcement model is that environmental feedback does not come in the form of reinforcement but, rather, in the form of changes in drive levels. Biological systems appear to compute their own reinforcement by utilizing learning mechanisms that compare current and recent drive levels. In this way, a drive-reinforcement learning mechanism requires no evaluative feedback from the environment. The environment simply provides sensory inputs, some of which function as drives, and when the drive levels change, it is hypothesized that neurons and nervous systems treat these changes in drive levels as reinforcement.

By evaluative feedback, an expression I have used above, I mean any kind of signal that requires the environment (actually, a "teacher" or "trainer" in the environment) to make some judgment about the performance of the learning system that is receiving the feedback. In an extreme case, that could mean that the teacher or trainer would have to know the desired response and would then inform the learning system of the direction and magnitude of its error. In a less extreme case, the teacher or trainer could utilize implicit or explicit criteria to form judgments about whether the learning system's performance was improving or not and then signal these evaluations of relative levels of performance to the learning system. Nonevaluative feedback, then, is any signal a learning system can generate for itself, without the aid of a teacher or trainer, simply by having an appropriate sensor with which to detect events in the environment.

Whether feedback comes to a learning system in the form of drives, reinforcers, or error signals has relevance with regard to two further questions: What should constitute the innate knowledge in a learning system, and what form should the innate knowledge take? A reinforcement, or a supervised, learning system will, innately, know how to utilize reinforcement signals or error signals to discover appropriate drives. A drive-reinforcement learning system, on the other hand, will begin with some primary drives in place and will then acquire additional drives, utilizing changes in the current drives as reinforcers. Biological systems appear to take the latter approach, beginning with some primary or innate drives and then building acquired drives on top of them.

This approach may offer a solution to a fundamental problem in connectionist modeling. A basic question has been that of how the network elements or neurons in a large, deep, multilayered network can learn to respond properly without direct feedback from a teacher, that is, without information about what their correct responses should have been at each step along the way. The answer suggested by drive-reinforcement learning theory, as outlined earlier, is to utilize whatever network drives (feedback loops) are already in place and then to treat changes in drive levels as reinforcers. In this way, reinforcement signals are always available locally (i.e., changes in neuronal drive levels can be computed locally), and, thus, there would appear to be no requirement for a teacher or critic at any level in the network. (This does not preclude the eventual evolution, at higher levels in a neural network, of global reinforcement centers that could aid the process of learning by providing overall direction. Global reinforcement centers in the limbic system and hypothalamus may function in just this way.) Additional theoretical work, including computer simulations of large, deep networks, will be required to test the idea that drivereinforcement learning mechanisms will enable multilayered networks to learn to model their environment appropriately without evaluative feedback from the environment.

Having examined the kinds of environmental feedback required by various classes of connectionist models, let us now consider the related question of what kinds of goals are implemented in these networks. In supervised learning systems, the goal is to minimize an error signal. In reinforcement learning systems, the goal may be to maximize a scalar associated with the reinforcement function. In drive-reinforcement learning systems, the goal may be to reduce drives, although, as discussed above, biological systems do not always appear to be reducing drives and, even if they are, the behavioral manifestations can be subtle and complex. Some of the subtleties and complexities may be due to the presence of global reinforcement centers at the level of the limbic system and hypothalamus. The effects of global reinforcement centers may, in part, have motivated proposals for reinforcement learning systems as models of nervous system function. At a still higher level, cognitive processing appears to have motivated the introduction of supervised learning systems as theoretical models. From this perspective, we see that the drive-reinforcement learning mechanism might reflect the neuronal level of nervous system function, with reinforcement and supervised learning mechanisms reflecting progressively higher levels of function. It would seem, then, that it is important to be clear about what level of nervous system function one is modeling. Furthermore, modeling higher levels of nervous system function may require taking into account the nature of the learning mechanisms that operate at lower levels.

With regard to drive reduction as the possible goal of biological systems and, perhaps, as the goal of drivereinforcement networks, one point that should be made is that drive reduction would seem to be the goal for drives that are implemented as negative feedback loops. Drives implemented as positive feedback loops would seem to support the goal of drive induction rather than drive reduction. With this having been said, it may then be observed 
that, in the case of biological systems, drive induction, as in the pursuit of prey, always seems to be followed by drive reduction, as in the consumption of prey. This may suggest a simple general principle for the design (or evolution) of drive-reinforcement networks: primary drives implemented as positive feedback loops should always lead, when activated, to the subsequent activation of primary drives that are implemented as negative feedback loops. If this principle is followed, then all drives will, ultimately, support the goal of drive reduction. This may help to ensure the stability of learning systems.

I have traversed the conceptual or theoretical territory of connectionist models twice now, once looking at the kinds of feedback various classes of models require from their environments and once looking at the nature of the goals implemented in these models. I want to make one more pass, in order to examine the algorithmic or heuristic character of various connectionist models.

Supervised learning mechanisms, in their most recent form, in which back-propagation techniques are utilized, have a certain appeal because of what I would suggest is their nearly algorithmic character. I mean this in the mathematical sense, in which an algorithm is defined to be a procedure that is guaranteed to produce a result, as distinguished from a heuristic, which, like a rule of thumb, may or may not produce the desired outcome. Backpropagating error-correction learning mechanisms utilize gradient descent techniques such that they provide, with some allowances for the problem of getting hung up on local peaks, an optimal solution to the problem confronting the network, the problem being to arrive at the best set of connection weights. Back-propagating errorcorrection networks become of interest, then, from a theoretical standpoint, irrespective of their biological relevance, because the models may represent optimal or near optimal solutions of certain problems. Even here, there may be difficulties though, because for the larger, deeper networks that many theorists are interested in, scaling up of back-propagating error-correction networks may pose an obstacle (Plaut, Nowlan, \& Hinton, 1986).

At any rate, if we consider that back-propagating errorcorrection networks have something of an algorithmic character, the other extreme might be connectionist networks that utilize random search techniques to identify reasonable values for the connection weights (e.g., see Barron, 1968). Random search techniques would seem to be about as far removed from an algorithmic character as a learning mechanism can get.

In between these two extremes are such classes of models as reinforcement and drive-reinforcement learning mechanisms that appear to have a heuristic character. For example, utilizing drives and reinforcers as the basis for learning may not guarantee correct results, but, on the average, such an approach to learning appears to be effective in the case of biological systems.

\section{Artificial Intelligence}

Fundamental to the process of learning in the case of the drive-reinforcement neuronal model is the temporal shaping of behavior. This is in contrast to the kinds of processes that occur in artificial intelligence, where the emphasis is placed on what might be called cognitive searching - "cognitive" because there is an emphasis on the rational and symbolic aspects of intelligence, and "searching" because there is an emphasis on selecting from a large number of possible behaviors. An implication of the drive-reinforcement neuronal model is that, fundamentally, natural intelligence and the learning mechanisms that support it do not involve symbols or searching but, rather, actions and shaping. Learned behavior is gradually shaped through experience to become more appropriate. This dynamic process yields associations that refine behavior that is already in place. Animals are continually "riding" a large number of feedback loops that reach through the animal and out into the environment. The more cognitively or symbolically oriented kinds of searching through large numbers of possibilities that humans sometimes engage in is, most likely, an emergent phenomenon that arises out of the internalization of a very large number of causal relations, this internalization being accomplished, it would seem, with something like a drive-reinforcement learning mechanism that temporally refines actions. Another way of saying this is that first we learn to grasp an object and then we learn to grasp a problem.

The comments I am making regarding artificial intelligence research apply as well, I feel, to cognitive science. There seems to be the view in both of these disciplines that memory, learning, and intelligence have to do, fundamentally, with cognition. However, doesn't natural intelligence have to do with action, emotion, and cognition? The drive-reinforcement neuronal model contains what may be a complete set of the fundamental elements that underlie intelligence, namely, outputs that reflect actions, inputs and changes in inputs that reflect drives and reinforcers, synaptic weights that represent knowledge, and changes in synaptic weights that represent learning. The seeds of action, emotion, and cognition appear to be present in the drive-reinforcement neuronal model.

In such areas of artificial intelligence research as image understanding and the related area of pattern recognition (although the latter is sometimes more closely associated with connectionist models than with mainstream artificial intelligence), the tendency has been to treat the temporal aspects of intelligent information processing as too difficult for current techniques to handle. (Some recent research constitutes exceptions to this statement.) Often, ways have been sought to automatically understand static scenes or to recognize spatial patterns. The temporal aspects of natural intelligence, associated with motion and with real-time information processing, in general, have frequently not been addressed in image understanding and pattern recognition research, the strategy seeming to be that these difficult issues will be addressed after these fields of research are more advanced. But if the temporal and, indeed, real-time aspects of natural intelligence turn out to be fundamental with regard to learning, as the drive-reinforcement model suggests, could it be that the 
goals of image understanding and pattern recognition research will be more easily achieved if the temporal or real-time aspects of intelligent information processing are confronted first rather than last?

Having discussed cognitive searching and its role in artificial intelligence, it may be useful at this point to comment on evolutionary models of learning, because such models also invoke search mechanisms in a fundamental way. Fogel, Owens, and Walsh (1966), Klopf and Gose (1969), and Holland (1975), for example, have proposed evolutionary models of learning in which alternative structures or behaviors are generated randomly or by some process that is more systematic than a purely random one, after which the alternatives are evaluated and the best are saved. Such an evolutionary process appears to be fundamentally different from a learning process. Fundamentally, learning does not appear to involve generating and evaluating alternatives. Rather, as discussed earlier, learning appears to involve the direct temporal shaping of behavior. Experienced causal relationships are internalized; that is, associations are formed directly as a result of the experience. For example, when a bell rings and food follows, animals form associations directly. No search process occurs. Of course, at a higher level, searching can be occurring. It can be seen that if an animal is exploring its environment and causes a bell to ring and then food follows, the consequences of the exploratory or search process may result in the direct temporal shaping of behavior. Direct temporal shaping of behavior may be occurring then at the most fundamental level and a search process may be occurring at a higher level.

In summary, it could be said that an implication of the drive-reinforcement model is that time is the teacher (that is to say, real time) and behavior or actions is what is taught. Ultimately, in a phylogenetically advanced organism like a human, knowledge acquisition, representation, and utilization become important too, and then a process like the one I am calling cognitive searching takes on increasing importance. However, it seems that this may have mislead artificial intelligence researchers and cognitive scientists, drawing their attention away from the underlying mechanisms that appear to have more to do with temporal shaping. Artificial intelligence researchers have, for example, sometimes been dismayed by the lack of common sense in the systems they have designed. Could it be that common sense derives from the operation of drives and reinforcers and from the kind of realtime embedding in the environment that is characteristic of biological systems?

\section{Adaptive Control Theory and Adaptive Signal Processing}

For several decades now, control theory has been successfully applied to the problems of analyzing and synthesizing automatic control systems. Adaptive control theory seeks to extend control system applications to cases in which adaptation or learning is required on the part of the automatic controller (e.g., see Chalam, 1987). In this way, control theory contacts the problem of learning in the context of engineering applications.

Related to the subject of adaptive control theory is adaptive signal processing (e.g., see Widrow \& Stearns, 1985). In both adaptive control and adaptive signal processing, it is sometimes assumed that a "desired response" or "training signal" is available with which the controller's or signal processor's actual output can be compared for the purpose of learning. Drive-reinforcement learning theory, as outlined earlier, suggests an alternative way to extend control theory or signal processing techniques for the case of learning, such that no knowledge of a desired response or training signal is required when the learning system is operating.

In the drive-reinforcement learning theory outlined earlier, network drives are fundamental. In control theory, negative feedback loops are fundamental. But network drives, as I have defined them, and negative feedback loops are one and the same thing. (One qualification: In biological systems, network drives may also occasionally be positive feedback loops.) One sees that drivereinforcement theory and control theory start on the same basis. It can then be seen that the drive-reinforcement model suggests a "natural" learning mechanism for control and signal processing systems. Although I am not aware of any adaptive control or signal processing systems using lagged derivatives of inputs and outputs as a basis for adaptation, such a learning mechanism would seem to constitute a straightforward extension of conventional control system and signal processing techniques.

The essence of the drive-reinforcement learning mechanism, in adaptive control theoretic terms, can be simply stated. A network of drive-reinforcement neurons, viewed as a control system, will interact with its environment through some set of positive and negative feedback loops. Pursuit of prey, for example, may involve positive feedback loops, as noted earlier, and avoidance of predators may involve negative feedback loops. At any given time, a biological system will be interacting with its environment through a set of actual positive and negative feedback loops that constitute its current primary and acquired drives and through a set of potential positive and negative feedback loops that constitute possible future acquired drives. Potential acquired drives will become actual if the inputs for the potential drives become active no more than $\tau$ time steps before any of the current actual drives change their levels of activity. In this way, what may be called a drive-reinforcement controller will learn to control its output not only to deliver more or less of a control signal (as current adaptive controllers do), but also to deliver the control signal sooner or later. That is to say, a drivereinforcement controller would be expected to modify not only the amplitudes of its responses, but also the timing.

\section{Memory and Learning}

Before concluding this discussion of some of the implications of the drive-reinforcement model, a few words should be said about memory and how it relates to leam- 
ing. As Squire (1986) noted, in phylogenetically old animals, such as invertibrates, what is learned takes the form of procedural memories. In phylogenetically recent animals, such as mammals, what is learned can also take the form of declarative memories. The distinction between procedural and declarative memories is that between skills and procedures, on the one hand, and specific facts and data, on the other.

The drive-reinforcement learning mechanism appears to be well suited for the laying down of procedural memories, because the learning mechanism treats time as a fundamental dimension, utilizing time derivatives of the neuronal inputs and outputs and correlating the derivatives across a temporal interval. If the drive-reinforcement learning mechanism should turn out to be the learning mechanism for acquiring procedural memories, could it also turn out to be the learning mechanism for acquiring declarative memories? To see how this could be a possibility, it may be necessary to consider the interaction of the brain's attention mechanism with the registration of sensory and other information in the cerebral cortex. The medial temporal cortex and, especially, the hippocampal formation and associated areas appear to be important with respect to declarative memories. Squire (1986) noted that the capacity for declarative memories reaches its greatest development in mammals in which these cortical structures are most fully elaborated. Given our tendency to remember that to which we attend, might it be that signals generated by the attention mechanism, the signals originating perhaps in the thalamic reticular formation (Klopf, 1982; Crick, 1984), interact with sensory and other information registering in the medial temporal cortex, such that the temporal relationships specified by the drive-reinforcement learning mechanism are satisfied and declarative memories result? In general, could the role of the attention mechanism in the laying down of both procedural and declarative memories be the induction of $\Delta y$ s at appropriate times relative to $\Delta x$ s so that the resulting synaptic weight changes yield learning?

\section{CONCLUDING REMARKS}

In the foreword to Olds's (1977) book, Drives and Reinforcements, Neal Miller remarks:

A fundamental step in the line of evolution leading to human behavior was the development of learning, a new process of adaptation that could occur far more rapidly within the lifetime of the individual instead of slowly during the evolution of the species. In determining which particular response will be performed and learned, the selective factor is reinforcement which, in turn, is closely related to the drives that are active at a given time. (p. v)

In this article, I have attempted to relate drives and reinforcers by means of a theoretical model of neuronal function. The model has been demonstrated to predict a wide range of classical conditioning phenomena. Implications of the model have been considered for the fields of animal learning theory, connectionist and neural network modeling, artificial intelligence research, adaptive control theory, and adaptive signal processing. It has been concluded that a real-time learning mechanism that does not require evaluative feedback from the environment may be fundamental to natural intelligence and that such a learning mechanism may have implications for artificial intelligence.

In addition to accomplishing experimental tests of the neuronal model, a useful next step may be to simulate networks of the proposed theoretical neurons to determine the properties of the networks, in general, and, in particular, to determine if instrumental conditioning phenomena emerge. Actually, in pursuing this theoretical work, it may be useful to simulate not only the neural network, but also a simplified organism controlled by the neural network and a simplified environment with which the organism is interacting. (See Barto \& Sutton, 1981b, for an example of how this kind of simulation can be carried out.) By means of such computer simulations of nervous systems, organisms, and environments, it may become possible to make behavioral observations on a mathematically well-defined network of drive-reinforcement neurons during the process of learning.

\section{REFERENCES}

Ackley, D. H., Hinton, G. H., \& Sejnowski, T. J. (1985). A leaming algorithm for Boltzmann machines. Cognitive Science, 9, 147-169. Anderson, J. A., Silverman, J. W., Ritz, S. A., Jones, R.S. (1977). Distinctive features, categorical perception, and probability learning: Some applications of a neural model. Psychological Review, 84, 413-451.

Ashton, A. B., Bitgood, S. C., \& Moore, J. W. (1969). Auditory differential conditioning of the rabbit nictitating membrane response: III. Effects of US shock intensity and duration. Psychonomic Science, $15,127-128$.

Ayres, J. J. B., Albert, M., Bombace, J. C. (1987). Extending conditioned stimuli before versus after unconditioned stimuli: Implications for real-time models of conditioning. Journal of Experimental Psychology: Animal Behavior Processes, 13, 168-181.

BAKER, T. W. (1968). Component strength in a compound CS as a function of number of acquisition trials. Joumal of Experimental Psychology, 79, 347-352.

Balsam, P. D., Schwartz, A. L. (1981). Rapid contextual conditioning in autoshaping. Joumal of Experimental Psychology: Animal Behavior Processes, 7, 382-393.

Baranyi, A., Ferher, O. (1978). Conditioned changes of synaptic transmission in the motor cortex of the cat. Experimental Brain Research, 33, 283-298.

Barany1, A., Ferher, O. (1981a). Intracellular studies on cortical synaptic plasticity: Conditioning effect of antidromic activation on test-EPSPs. Experimental Brain Research, 41, 124-134.

Baranyi, A., Ferher, O. (1981b). Long-term facilitation of excitatory synaptic transmission in single motor cortical neurones of the cat produced by repetitive pairing of synaptic potentials and action potentials following intracellular stimulation. Neuroscience Letters, 23, 303-308.

Baryanyi, A., Ferher, O. (1981c). Synaptic facilitation requires paired activation of convergent pathways in the neocortex. Nature (London), 290, 413-415.

BARRON, R. L. (1968). Self-organizing and learning control systems. In H. L. Ostreicher \& D. Moore (Eds.), Cybernetic problems in bionics (pp. 147-203). New York: Gordon and Breach. 
BARTo, A. G. (1985). Learning by statistical cooperation of selfinterested neuron-like computing elements. Human Neurobiology, 4, 229-256.

Barto, A. G., \& ANandan, P. (1985). Pattern recognizing stochastic learning automata. IEEE Transactions on Systems, Man, \& Cybernetics, SMC-15, 360-374.

BArTo, A. G., \& ANDERson, C. W. (1985). Structural learning in connectionist systems. In Proceedings of the Seventh Annual Conference of the Cognitive Science Society (pp. 43-53). Hillsdale, NJ: Erlbaum.

Barto, A. G., \& SuTton, R. S. (1981a). Goal seeking components for adaptive intelligence: An initial assessment (Tech. Rep. No. 811070). Wright-Patterson Air Force Base, OH: Air Force Wright Aeronautical Laboratories. (DTIC Report AD 101476 available from the Defense Technical Information Center, Cameron Station, Alexandria, VA 22304-6145)

Barto, A. G., \& Sutton, R. S. (1981b). Landmark leaming: An illustration of associative search. Biological Cybermetics, 42, 1-8.

BArto, A. G., \& SutTon, R. S. (1982). Simulation of anticipatory responses in classical conditioning by a neuron-like adaptive element. Behavioural Brain Research, 4, 221-235.

Barto, A. G., Sutton, R. S., \& Anderson, C. W. (1983). Neuronlike elements that can solve difficult learning control problems. IEEE Transactions on Systems, Man, \& Cybernetics, SMC-13, 835-846.

BAUDRY, M. (1987). Activity-dependent regulation of synaptic transmission and its relationship to learning (Group Report, Dahlem Workshop). In J.-P. Changeux \& M. Konishi (Eds.), The neural and molecular bases of learning (pp. 152-175). New York: Wiley.

BERLYNE, D. E. (1973). The vicissitudes of aplopathematic and thelematoscopic pneumatology (or the hydrography of hedonism). In D. E. Berlyne \& K. B. Madsen (Eds.), Pleasure, reward, preference: Their nature, determinants, and role in behavior (pp. 1-33). New York: Academic Press.

BINDRA, D. (1968). Neuropsychological interpretation of the effects of drive and incentive-motivation on general activity and instrumental behavior. Psychological Review, 75, 1-22.

BINDRA, D. (1976). A theory of intelligent behavior. New York: Wiley.

BINDRA, D. (1978). How adaptive behavior is produced: A perceptualmotivational alternative to response-reinforcement. Behavioral \& Brain Sciences, 1, 41-91.

Blazis, D. E. J., Desmond, J. E., Moore, J. W., \& Bertheir, N. E. (1986). Simulation of the classically conditioned nictitating membrane response by a neuron-like adaptive element: $A$ real-time variant of the Sutton-Barto model. In Proceedings of the Eighth Annual Conference of the Cognitive Science Society (pp. 176-186). Hilladale, NJ: Erlbaum.

BLAZIS, D. E. J., \& MOORE, J. W. (1987). Simulation of a classically conditioned response: Components of the input trace of a cerebellar neural network implementation of the Sutton-Barto-Desmond model (Tech. Rep. No. 87-74). Amherst: University of Massachusetts, Computer and Information Science Department.

Buss, T. V. P., \& Lomo, T. (1973). Long-lasting potentiation of synaptic transmission in the dentate area of the anaesthetised rabbit following stimulation of the perforant path. Joumal of Physiology (London), 232, 331-356.

Bolles, R. C. (1967). Theory of motivation. New York: Harper \& Row. Bolles, R. C. (1972). Reinforcement, expectancy and learning. Psychological Review, 79, 394-409.

Booker, L. B. (1982). Intelligent behavior as an adaptation to the task environment (Doctoral dissertation, University of Michigan, 1982). Dissertation Abstracts International, 43, 469B.

Brown, P. L., Jenkins, H. M. (1968). Autoshaping of the pigeon's key peck. Journal of the Experimental Analysis of Behavior, 11, 1-8.

BurkHARDT, P. E., \& AYRES, J. J. B. (1978). CS and US duration effects in one-trial simultaneous fear conditioning as assessed by conditioned suppression of licking in rats. Animal Learning \& Behavior, 6, 225-230.

Byrne, J. H. (1987). Cellular analysis of associative learning. Physiological Reviews, 67, 329-439.

CALVIN, W. H. (1975). Generation of spike trains in CNS neurons. Brain Research, 84, 1-22.
Carew, T. J., Hawkins, R. D., Abrams, T. W., \& Kandel, E. R. (1984). A test of Hebb's postulate at identified synapses which mediate classical conditioning in Aplysia. Journal of Neuroscience, 4, 1217-1224.

CAREW, T. J., \& SAHLEY, C. L. (1986). Invertebrate learning and memory: From behavior to molecules. Annual Review of Neuroscience, 9, 435-487.

Carew, T. J., Walters, E. T., \& Kandel, E. R. (1981). Classical conditioning in a simple withdrawal reflex in Aplysia Califormica. Journal of Neuroscience, 1, 1426-1437.

Chalam, V. V. (1987). Adaptive control systems. New York: Marcel Dekker.

Cohen, M. A., \& Grossberg, S. (1983). Absolute stability of global pattern formation and parallel memory storage by competitive neural networks. IEEE Transactions on Systems, Man, \& Cybernetics, SMC13, 815-826.

Couvillon, P. A., \& Bitterman, M. E. (1982). Compound conditioning in honeybees. Journal of Comparative Psychology, 96, 192-199.

CRICK, F. (1984). Function of the thalamic reticular complex: The searchlight hypothesis. Proceedings of the National Academy of Sciences USA, 81, 4586-4590.

Delgado, J. M. R., Roberts, W. W., a Miller, N. E. (1954). Learning motivated by electrical stimulation of the brain. American Journal of Physiology, 179, 587-593.

Dickinson, A., \& Mackintosh, N. J. (1978). Classical conditioning in animals. Annual Review of Psychology, 29, 587-612.

Dolan, J. C., Shishimi, A., \& Wagner, A. R. (1985). The effects of signaling the US in backward conditioning: A shift from excitatory to inhibitory learning. Animal Learning \& Behavior, 13, 209-214.

Donegan, N. H., \& Wagner, A. R. (1987). Conditioned diminution and facilitation of the UR: A sometimes opponent-process interpretation. In I. Gormezano, W. F. Prokasy, \& R. F. Thompson (Eds.), Classical conditioning (pp. 339-369). Hillsdale, NJ: Erlbaum.

ECCLEs, J. C. (1964). The physiology of synapses. New York: Academic Press.

Estes, W. K. (1950). Toward a statistical theory of learning. Psychological Review, 57, 94-107.

FARLEY, B. G., \& ClaRK, W. A. (1954). Simulation of self-organizing systems by digital computer. IRE Transactions on Information Theory, PGIT-4, 76-84.

FARLeY, J., \& AlKon, D. L. (1985). Cellular mechanisms of learning, memory, and information storage. Annual Review of Psychology, 36, 419-494.

Feldman, J. A. (Ed.) (1985). Special issue on connectionist models and their applications. Cognitive Science, 9.

FlaHERTY, C. F. (1985). Animal learning and cognition. New York: Knopf.

Flaherty, C. F., Hamilton, L. W., Gandelman, R. J., \& Spear, N. E. (1977). Learning and memory. Chicago: Rand McNally.

FoGEL, L. J., OWENS, A. J., \& WALSH, M. J. (1966). Artifical intelligence through simulated evolution. New York: Wiley.

FowLER, H. (1971). Implications of sensory reinforcement. In R. Glaser (Ed.), The nature of reinforcement (pp. 151-195). New York: Academic Press.

Freud, S. (1964). Untitled paper. In J. Strachey (Ed.), The standard edition of the complete psychological works of Sigmund Freud (Vol. 1, pp. 281-387). New York: MacMillan. (Original work written in 1895)

Frey, P. W., Ross, L. E. (1968). Classical conditioning of the rabbit eyelid response as a function of interstimulus interval. Journal of Comparative \& Physiological Psychology, 65, 246-250.

FukUSHIMA, K. (1980). Neocognitron: A self-organizing neural network model for a mechanism of pattern recognition unaffected by shift in position. Biological cybermetics, 36, 193-202.

FUKUSHIMA, K., \& MIYAKE, S. (1982). Neocognition: A self-organizing neural network model for a mechanism of visual pattern recognition. In S. Amari \& M. A. Arbib (Eds.), Competition and cooperation in neural nets (pp. 267-285). New York: Springer-Verlag.

FuXe, K. (1965). Acta Physiologica Scandinavica, 64(Suppl. 247), 37-84. 
Gelperin, A., Hopfield, J. J., \& TAnk, D. W. (1985). The logic of Limax learning. In A. Selverston (Ed.), Model neural networks and behavior (pp. 237-261). New York: Plenum Press.

Gluck, M. A., Thompson, R. F. (1987). Modeling the neural substrates of associative learning and memory: A computational approach. Psychological Review, 94, 176-191.

Gormezano, I. (1972). Investigations of defense and reward conditioning in the rabbit. In A. H. Black \& W. F. Prokasy (Eds.), Classical conditioning II: Current research and theory (pp. 151-181). New York: Appleton.

Gormezano, I., Kehoe, E. J., Marshall, B. S. (1983). Twenty years of classical conditioning research with the rabbit. In J. M. Sprague \& A. N. Epstein (Eds.), Progress of psychobiology and physiological psychology (pp. 198-274). New York: Academic Press.

Gould, J. L. (1986). The biology of learning. Annual Review of Psychology, 37, 163-192.

GRAY, J. A. (1975). Elements of a two-process theory of leaming. New York: Academic Press.

Grossberg, S. (1982). Studies of mind and brain. Boston: Reidel.

Grossberg, S. (Ed.) (1987). The adaptive brain (Vols. 1 \& 2). New York: North-Holland.

GuTHRIE, E. R. (1935). The psychology of leaming. New York: Harper \& Row.

HAMpson, S. E. (1984). A neural model of adaptive behavior (Doctoral dissertation, University of California, Irvine, 1983). Dissertation Abstracts Intemational, 44, 3457B.

Hawkins, R. D., Abrams, T. W., Carew, T. J., Kandel, E. R. (1983). A cellular mechanism of classical conditioning in Aplysia: Activity-dependent amplification of presynaptic facilitation. Science, 219, 400-405.

Hawkins, R. D., Carew, T. J., \& Kandel, E. R. (1986). Effects of interstimulus interval and contingency on classical conditioning of the Aplysia siphon withdrawal reflex. Joumal of Neuroscience, 6 , 1695-1701.

HAwKINS, R. D., \& KANDEL, E. R. (1984). Steps toward a cell-biological alphabet for elementary forms of learning. In G. Lynch, J. L. McGaugh, \& N. M. Weinberger (Eds.), Neurobiology of learning and memory (pp. 385-404). New York: Guilford Press.

HeBs, D. O. (1949). The organization of behavior. New York: Wiley. Helson, H. (1964). Adaptation-level theory. New York: Harper \& Row.

HETH, C. D. (1976). Simultaneous and backward fear conditioning as a function of number of CS-UCS pairings. Journal of Experimental Psychology: Animal Behavior Processes, 2, 117-129.

Hilgard, E. R., Bower, G. H. (1975). Theories of learning (4th ed.). Englewood Cliffs, NJ: Prentice-Hall.

Hinton, G. E., \& Anderson, J. A. (Eds.) (1981). Parallel models of associative memory. Hillsdale, $\mathrm{NJ}$ : Erlbaum.

Hinton, G. E., SEJNowskı, T. J. (1986). Learning and relearning in Boltzmann machines. In E. E. Rumelhart \& J. L. McClelland (Eds.), Parallel distributed processing: Explorations in the microstructure of cognition: Vol. 1. Foundations (pp. 282-317). Cambridge, MA: Bradford Books/MIT Press.

Hinton, G. E., Sejnowski, T. J., \& ACKLey, D. H. (1984). Boltzmann machines: Constraint satisfaction networks that leam (Tech. Rep. No. CMU-CS-84-119). Pittsburgh: Carnegie-Mellon University, Computer Science Department.

Holland, J. H. (1975). Adaptation in natural and artificial systems. Ann Arbor: University of Michigan Press.

Holland, J. H. (1986). Escaping brittleness: The possibilities of generalpurpose learning algorithms applied to parallel rule-based systems. In R. S. Michalski, J. G. Carbonell, \& T. M. Mitchell (Eds.), Machine leaming: Artificial intelligence approach (Vol. 2, pp. 593-623). Los Altos, CA: Morgan-Kaufmann.

HoPFIELD, J. J. (1982). Neural networks and physical systems with emergent collective computational abilities. Proceedings of the National Academy of Sciences USA, 79, 2554-2558.

HopFIELD, J. J. (1984). Neurons with graded response have collective computational properties like those of two-state neurons. Proceedings of the National Academy of Sciences USA, 81, 3088-3092.

Hopfield, J. J., \& TANK, D. W. (1985). Neural computation of decisions in optimization problems. Biological Cybermetics, 52, 141-152.
Hopfield, J. J., * TANK, D. W. (1986). Computing with neural circuits: A model. Science, 233, 625-633.

Hull, C. L. (1943). Principles of behavior. New York: AppletonCentury-Crofts.

Hull, C. L. (1952). A behavior system: An introduction to behavior theory conceming the individual organism. New Haven: Yale University Press.

Iто, M. (1984). The modifiable neuronal network of the cerebellum. Japanese Journal of Physiology, 34, 781-792.

ITo, M. (1986). Long-term depression as a memory process in the cerebellum. Neuroscience Research, 3, 531-539.

Ito, M., Sakurai, M., \& Tongroach, P. (1982). Climbing fibreinduced depression of both mossy fibre responsiveness and glutamate sensitivity of cerebellar Purkinje cells. Joumal of Physiology (London), 324, 113-134.

Jenkins, H. M., \& Moore, B. R. (1973). The form of the autoshaped response with food or water reinforcers. Joumal of the Experimental Analysis of Behavior, 20, 163-181.

Kamin, L. J. (1965). Temporal and intensity characteristics of the conditioned stimulus. In W. F. Prokasy (Ed.), Classical conditioning: A symposium (pp. 118-147). New York: Appleton-Century-Crofis.

KAMIN, L. J. (1968). Attention-like processes in classical conditioning. In M. R. Jones (Ed.), Miami Symposium on the Prediction of Behavior: Aversive stimulation (pp. 9-31). Miami: University of Miami Press.

Kamin, L.. J. (1969). Predictability, surprise, attention and conditioning. In B. A. Campbell \& R. M. Church (Eds.), Punishment and aversive behavior (pp. 279-296). New York: Appleton-Century-Crofis.

Kandel, E. R., SPEnCER, W. A. (1968). Cellular neurophysiological approaches in the study of learning. Physiological Reviews, 48, 65-134.

KEENE, J. J. (1973). Reward-associated excitation and pain-associated inhibition lasting seconds in rat medial pallidal units. Experimental Neurology, 49, 97-114.

KeHOE, E. J. (in press). A layered network model of associative learning: Learning-to-learn and configuration. Psychological Review.

Kelso, S. R., \& Brown, T. H. (1986). Differential conditioning of associative synaptic enhancement in hippocampal brain slices. Science, 232, 85-87.

Kelso, S., Ganong, A., \& Brown, T. H. (1986). Hebbian synapses in hippocampus. Proceedings of the National Academy of Sciences USA, 83, 5326-5330.

KLOPF, A. H. (1972). Brain function and adaptive systems-A heterostatic theory (Rep. No. 133 [AFCRL-72-0164]). L. G. Hanscom Field, Bedford, MA: Air Force Cambridge Research Laboratories. (DTIC Report AD 742259, available from the Defense Technical Information Center, Cameron Station, Alexandria, VA 22304-6145)

KLOPF, A. H. (1975). A comparison of natural and artificial intelligence. Association for Computing Machinery Special Interest Group on Artificial Intelligence Newsletter, No. 52, 11-13.

KLOPF, A. H. (1979). Goal-seeking systems from goal-seeking components; Implications for AI. Cognition \& Brain Theory Newsletter, 3, 54-62.

KLOPF, A. H. (1982). The hedonistic neuron: A theory of memory, learning, and intelligence. New York: Hemisphere.

KLOPF, A. H. (1986). A drive-reinforcement model of single neuron function: An alternative to the Hebbian neuronal model. In J. S. Denker (Ed.), AIP Conference Proceedings 151: Neural networks for computing (pp. 265-270). New York: American Institute of Physics.

KLOPF, A. H., \& GosE, E. (1969). An evolutionary pattern recognition network. IEEE Transactions on Systems Science and Cybemetics, SSC5, 247-250.

KoHonen, T. (1977). Associative memory: A system theoretic approach. New York: Springer-Verlag.

KOHONEN, T. (1984). Self-organization and associative memory. New York: Springer-Verlag.

KONORSKI, J. (1967). Integrative activity of the brain: An interdisciplinary approach. Chicago: University of Chicago Press.

Kosko, B. (1986). Differential Hebbian learning. In J. S. Denker (Ed.), AIP Conference Proceedings 15I: Neural networks for computing (pp. 277-282). New York: American Institute of Physics. 
Kremer, E. F. (1978). The Rescorla-Wagner model: Losses in associative strength in compound conditioned stimuli. Joumal of Experimental Psychology: Animal Behavior Processes, 4, 22-36.

LARSON, J., \& LYNCH, G. (1986). Induction of synaptic potentiation in hippocampus by patterned stimulation involves two events. Science, 232, 985-988.

LE CUN, Y. (1985). Une procédure d'apprentissage pour réseau a seuil asymétrique [A learning procedure for an asymmetric threshold network]. Proceedings of Cognitiva, 85, 559-604.

LEDERHENDLER, I., ALKON, D. L. (1986). Temporal specificity of the CS-UCS interval for Hermissenda Pavlovian conditioning. Society for Neuroscience Abstracts, 12(Pt. 1), 40.

Levine, D. S. (1983). Neural population modeling and psychology: A review. Mathematical Biosciences, 66, 1-86.

LEVY, W. B. (1985). Associative changes at the synapse: LTP in the hippocampus. In W. B. Levy, J. A. Anderson, \& S. Lehmkuhle (Eds.), Synaptic modification, neuron selectivity, and nervous system organization (pp. 5-33). Hillsdale, NJ: Erlbaum.

LEVY, W. B., \& Desmond, N. L. (1985). The rules of elemental synaptic plasticity. In W. B. Levy, J. A. Anderson, \& S. Lehmkuhle (Eds.), Synaptic modification, neuron selectivity, and nervous system organization (pp. 105-121). Hillsdale, NJ: Erlbaum.

LyNCh, J. C., Mountcastle, V. B., Talbot, W. H., YIN, T. C. T. (1977). Parietal lobe mechanisms for directed visual attention. Journal of Neurophysiology, 40, 362-389.

MaCkIntosh, N. J. (1974). The psychology of animal leaming. New York: Academic Press.

MACKINTOSH, N. J. (1975). A theory of attention: Variations in the associability of stimuli with reinforcement. Psychological Review, 82, 276-298.

MAhoney, W. J., Ayres, J. J. B. (1976). One-trial simultaneous and backward fear conditioning as reflected in conditioned suppression of licking in rats. Animal Learning \& Behavior, 4, 357-362.

MCALlister, W. R. (1953). Eyelid conditioning as a function of the CS-US interval. Journal of Experimental Psychology, 45, 417-422.

McClelland, D. C., Atkinson, J. W., Clark, R. A., \& Lowell, E. L. (1953). The achievement motive. New York: Appleton.

McClelland, J. L., \& Rumelhart, D. E. (Eds.) (1986). Parallel distributed processing: Explorations in the microstructure of cognition: Vol. 2. Psychological and biological models. Cambridge, MA: Bradford Books/MIT Press.

MCCulLOCH, W. S., P PrTs, W. (1965). A logical calculus of the ideas immanent in nervous activity. In W. S. McCulloch (Ed.), Embodiments of mind (pp. 19-39). Cambridge, MA: MIT Press. (Reprinted from Bulletin of Mathematical Biophysics, 1943, 5, 115-137)

McNaughton, B. L., Barnes, C. A., \& Rao, G. (1984). Presynaptic versus postsynaptic control over long-term synaptic enhancement. In G. Lynch, J. L. McGaugh, \& N. M. Weinberger (Eds.), Neurobiology of learning and memory (pp. 466-469). New York: Guilford Press.

Miller, N. E. (1951). Learnable drives and rewards. In S. S. Stevens (Ed.), Handbook of experimental psychology (pp. 435-472). New York: Wiley.

Miller, R. R., BAlaz, M. A. (1981). Differences in adaptiveness between classically conditioned responses and instrumentally conditioned responses. In N. E. Spear \& R. R. Miller (Eds.), Information processing in animals: Memory mechanisms (pp. 49-80). Hillsdale, NJ: Erlbaum.

MINSKY, M. L. (1954). Theory of neural-analog reinforcement systems and its application to the brain-model problem. Unpublished doctoral dissertation, Princeton University.

Mis, F. W., MoORe, J. W. (1973). Effect of preacquisition UCS exposure on classical conditioning of the rabbit's nictitating membrane response. Leaming \& Motivation, 4, 108-114.

MishKin, M., \& PeTru, H. L. (1984). Mernories and habits: Some implications for the analysis of learning and retention. In L. R. Squire \& N. Butters (Eds.), Neuropsychology of memory (pp. 287-296). New York: Guilford Press.

Mogenson, G. J., \& Phillups, A. G. (1976). Motivation: A physiological construct in search of a physiological substrate. In J. M. Sprague \& A. N. Epstein (Eds.), Progress in psychobiology and physiological psychology (pp. 189-243). New York: Academic Press.
MoORE, J. W. (1979). Brain processes and conditioning. In A. Dickinson \& R. A. Boakes (Eds.), Mechanisms of leaming and motivation: A memorial volume to Jerzy Konorski (pp. 111-142). Hillsdale, NJ: Erlbaum.

Moore, J. W., Desmond, J. E., Berthier, N. E., Blazis, D. E. J., SutTon, R. S., BARTo, A. G. (1986). Simulation of the classically conditioned nictitating membrane response by a neuron-like adaptive element: Response topography, neuronal firing, and interstimulus intervals. Behavioural Brain Research, 21, 143-154.

Moore, J. W., \& Gormezano, I. (1977). Classical conditioning. In M. H. Marx \& M. E. Bunch (Eds.), Fundamentals and applications of leaming (pp. 87-120). New York: Macmillan.

MOORE, J. W., STICKNEY, K. J. (1980). Formation of attentionalassociative networks in real time: Role of the hippocampus and implications for conditioning. Physiological Psychology, 8, 207-217. MOORE, J. W. , STICKNEY, K. J. (1985). Antiassociations: Conditioned inhibition in attentional-associative networks. In R. R. Miller \& N. E. Spear (Eds.), Information processing in animals: Conditioned inhibition (pp. 209-232). Hillsdale, NJ: Erlbaum.

MORRIS, R., B BAER, M. (1984). Does long-term potentiation/synaptic enhancement have anything to do with learning or memory? In L. R. Squire \& N. Butters (Eds.), Neuropsychology of memory (pp. 521-535). New York: Guilford Press.

MOWRER, O. H. (1960). Leaming theory and behavior. New York: Wiley. (Krieger Edition, 1973)

Mpitsos, G. J., Coluns, S. D., \& McClellan, A. D. (1978). Leaming: A model system for physiological studies. Science, 199, 497-506.

OLDS, J. (1977). Drives and reinforcements: Behavioral studies of hypothalamic functions. New York: Raven Press.

OLDS, J., M MiLner, P. (1954). Positive reinforcement produced by electrical stimulation of septal area and other regions of rat brain. Journal of Comparative \& Physiological Psychology, 47, 419-427.

PARKer, D. B. (1982). Leaming logic (Invention Report No. 581-64, File 1). Stanford, CA: Stanford University, Office of Technology Licensing.

PARKer, D. B. (1985). Learning-logic (Tech. Rep. No. 47). Cambridge, MA: Massachusetts Institute of Technology, Center for Computational Research in Economics and Management Science.

Pavlov, I. P. (1927). Conditioned reflexes. Oxford: Oxford University Press. (Dover Edition, 1960)

Pavlov, I. P. (1928). Lectures on conditioned reflexes. New York: International Publishers.

Plaut, D. C., Nowlan, S. J., Hinton, G. E. (1986). Experiments on learning by back propagation (Tech. Rep. No. CMU-CS-86-126). Pittsburgh: Carnegie-Mellon University, Department of Computer Science.

PremaCk, D. (1959). Toward empirical behavior laws: I. Positive reinforcement. Psychological Review, 66, 219-233.

RashevsKY, N. (1938). Mathematical biophysics. Chicago: University of Chicago Press.

Reiss, S., W Waner, A. R. (1972). CS habituation produces a "latent inhibition effect"' but no active conditioned inhibition. Leaming \& Motivation, 3, 237-245.

Rescorla, R. A. (1969). Pavlovian conditioned inhibition. Psychological Bulletin, 72, 77-94.

Rescorla, R. A. (1971a). Summation and retardation tests of latent inhibition. Joumal of Comparative \& Physiological Psychology, 75, 77-81.

Rescorla, R. A. (1971b). Variation in the effectiveness of reinforcement and nonreinforcement following prior inhibitory conditioning. Leaming \& Motivation, 2, 113-123.

Rescorla, R. A. (1980). Pavlovian second-order conditioning: Studies in associative leaming. New York: Erlbaum/Wiley.

Rescorla, R. A. (1988). Behavioral studies of Pavlovian conditioning. Annual Review of Neuroscience, 11, 329-352.

Rescorla, R. A., WAGNer, A. R. (1972). A theory of Pavlovian conditioning: Variations in the effectiveness of reinforcement and nonreinforcement. In A. H. Black \& W. F. Prokasy (Eds.), Classical conditioning II: Current research and theory (pp. 64-99). New York: Appleton-Century-Crofts.

RoBERTs, E. (1980). Epilepsy and antiepileptic drugs: A speculative 
synthesis. In G. H. Glaser, J. K. Penry, \& D. M. Woodbury (Eds.), Antiepileptic drugs: Mechanisms of action (pp. 667-713). New York: Raven Press.

RoLss, E. T. (1987). Information representation, processing, and storage in the brain: Analysis at the single neuron level. In J.-P. Changeux \& M. Konishi (Eds.), The neural and molecular bases of learning (pp. 503-540). New York: Wiley.

Rosenblatt, F. (1962). Principles of neurodynamics. New York: Spar$\tan$ Books.

Rumelhart, D. E., Hinton, G. E., \& Willuams, R. J. (1985). Leaming internal representations by error propagation (ICS Report No. 8506). San Diego: University of California, Institute for Cognitive Science.

Rumelhart, D. E., Hinton, G. E., \& Williams, R. J. (1986). Learning internal representations by error propagation. In D. E. Rumelhart \& J. L. McClelland (Eds.), Parallel distributed processing: Explorations in the microstructure of cognition. Vol. 1: Foundations (pp. 318-364). Cambridge, MA: Bradford Books/MIT Press.

Rumelhart, D. E., \& MCClelland, J. L. (Eds.) (1986). Parallel distributed processing: Explorations in the microstructure of cognition. Vol. 1: Foundations. Cambridge, MA: Bradford Books/MIT Press.

RusselL, I. S. (1966). Animal learning and memory. In D. Richter (Ed.), Aspects of learning and memory (pp. 121-171). New York: Basic Books.

SAmuel, A. L. (1963). Some studies in machine learning using the game of checkers. In E. A. Feigenbaum \& J. Feldman (Eds.), Computers and thought. New York: McGraw-Hill. (Reprinted from IBM Journal on Research \& Development, I959, 3, 210-229)

SChmAJUK, N. A., \& MoORE, J. W. (1985). Real-time attentional models for classical conditioning and the hippocampus. Physiological Psychology, 13, 278-290.

SChMAJUK, N. A., Moore, J. W. (1987). Two attentional models of classical conditioning: Variations in CS effectiveness revisited (Tech. Rep. No. 87-29). Amherst, MA: University of Massachusetts, Computer and Information Science Department.

SCHWARTZ, B. (1978). Psychology of leaming and behavior. New York: Norton.

SCHWARTZ, B. (1984). Psychology of learning and behavior (2nd ed.). New York: Norton.

Smith, M. C., Coleman, S. R., Gormezano, I. (1969). Classical conditioning of the rabbit's nictitating membrane response. Journal of Comparative \& Physiological Psychology, 69, 226-231.

Solomon, P. R., Brennan, G., MOORE, J. W. (1974). Latent inhibition of the rabbit's nictitating membrane response as a function of CS intensity. Bulletin of the Psychonomic Society, 4, 445-448.

Solomon, R. L. (1981). Prologue. In N. E. Spear \& R. R. Miller (Eds.), Information processing in animals: Memory mechanisms. Hillsdale, NJ: Erlbaum.

SPENCE, K. W. (1956). Behavior theory and conditioning. New Haven: Yale University Press.

Squire, L. R. (1986). Mechanisms of memory. Science, 232, 1612-1619.

Stein, L., \& BelluzzI, J. D. (1988). Operant conditioning of individual neurons. In M. Commons, R. Church, J. Stellar, \& A. Wagner (Eds.), Quantitative analyses of behavior: Vol. 7. Biological determinants of reinforcement and memory (pp. 249-264). Hillsdale, NJ: Erlbaum.

Stein, L., Wise, C. D., \& BelluzzI, J. D. (1977). Neuropharmacology of reward and punishment. In L. L. Iverson, S. D. Iverson, \& S. H. Snyder (Eds.), Handbook of psychopharmacology (Vol. 8, pp. 25-53). New York: Plenum Press.

Sutherland, N. S., \& Mackintosh, M. J. (1971). Mechanisms of animal discrimination learning. New York: Academic Press.

Surron, R. S. (1984). Temporal credit assignment in reinforcement learning. Unpublished doctoral dissertation, University of Massachusetts, Amherst.

SutToN, R. S. (1987). Leaming to predict by the methods of temporal differences (Tech. Rep. No. 87-509.1). Waltham, MA: GTE Laboratories.
Sutton, R. S., \& Barto, A. G. (1981). Toward a modern theory of adaptive networks: Expectation and prediction. Psychological Review, 88, $135-170$.

SutTon, R. S., \& BARTo, A. G. (1987). A temporal-difference model of classical conditioning (Tech. Rep. No. 87-509.2). Waltham, MA: GTE Laboratoreis.

Tesauro, G. (1986). Simple neural models of classical conditioning. Biological Cybernetics, 55, 187-200.

Thompson, R. F. (1976). The search for the engram. American Psychologist, 31, 209-227.

Thompson, R. F., Berger, T. W., \& MAdDen, J., IV. (1983). Cellular processes of learning and memory in the mammalian CNS. Annual Review of Neuroscience, 6, 447-491.

Thompson, R. F., McCormick, D. A., Lavond, D. G., Clark, G. A., KeTtNer, R. E., \& MAUK, M. D. (1983). The engram found? Initial localization of the memory trace for a basic form of associative learning. In J. M. Sprague \& A. N. Epstein (Eds.), Progress in psychobiology and physiological psychology (pp. 167-196). New York: Academic Press.

ThORNDIKE, E. L. (1911). Animal intelligence. New York: MacMillan. TOATES, F. (1985). Psychobiology [Review of The neurobiology of motivation and reward by J. R. Stellar \& E. Stellar]. Science, 229, 962-963.

W AGNER, A. R. (1969). Stimulus-selection and a "modified continuity theory." In G. H. Bower \& J. T. Spence (Eds.), The psychology of learning and motivation (Vol. 3, pp. 000-000). New York: Academic Press.

WAGNER, A. R. (1971). Elementary associations. In H. H. Kendler \& J. T. Spence (Eds.), Essays in neobehaviorism: A memorial volume to Kenneth W. Spence (pp. 187-213). New York: Appleton-CenturyCrofts.

WAGNER, A. R. (1981). SOP: A model of automatic memory processing in animal behavior. In N. E. Spear \& R. R. Miller (Eds.), Information processing in animals: Memory mechanisms (pp. 5-47). Hillsdale, NJ: Erlbaum.

Wagner, A. R., Logan, F. A., Haberlandt, K., \& Price, T. (1968). Stimulus selection in animal discrimination learning. Joumal of Experimental Psychology, 76, 171-180.

WAGNER, A. R., \& TERRY, W. S. (1975). Backward conditioning to a CS following an expected vs. a surprising UCS. Animal Learning \& Behavior, 3, 370-374.

W ALTERS, E. T., BYRNE, J. H. (1983). Associative conditioning of single neurons suggests a cellular mechanism for learning. Science, 219, 405-408.

WERBOS, P. J. (1974). Beyond regression: New tools for prediction and analysis in the behavioral sciences. Unpublished doctoral dissertation, Harvard University, Cambridge, MA.

WIDRow, B. (1962). Generalization and information storage in networks of adaline neurons. In M. C. Yovits, G. T. Jacobi, \& G. D. Goldstein (Eds.), Self-organizing systems - 1962 (pp. 435-461). Washington, DC: Spartan Books.

Widrow, B., Gupta, N. K., \& Maitra, S. (1973). Punish/reward: Learning with a critic in adaptive threshold systems. IEEE Transactions on Systems, Man, \& Cybernetics, SMC-5, 455-465.

WidRow, B., STEARNS, S. D. (1985). Adaptive signal processing. Englewood Cliffs, NJ: Prentice-Hall.

Williams, R. J. (1986). Reinforcement leaming in connectionist networks: A mathematical analysis (Tech. Rep. No. 8605). University of California, San Diego: Institute for Cognitive Science.

WILluams, R. J. (1987). Reinforcement-learning connectionist systems (Tech. Rep. No. NU-CCS-87-3). Boston, MA: Northeastern University.

WITTEN, I. H. (1977). An adaptive optimal controller for discrete-time Markov environments. Information \& Control, 34, 286-295.

Woody, C. D. (1982). Memory, learning and higher function. New York: Springer-Verlag.

Woody, C. D. (1986). Understanding the cellular basis of memory and learning. Annual Review of Psychology, 37, 433-493.

ZIPSER, D. (1986). A model of hippocampal learning during classical conditioning. Behavioral Neuroscience, 100, 764-776. 


\section{APPENDIX \\ Parameter Specifications for the Computer Simulations of the Neuronal Models}

\section{Drive-Reinforcement Model}

Learning rate constants: $c_{1}=5.0, c_{2}=3.0, c_{3}=1.5$, $c_{4}=0.75, c_{s}=0.25(\tau=5)$.

CS initial synaptic weight values [i.e., $w_{l}(t)$ at $\left.t=0\right]:+0.1$ (excitatory weights), -0.1 (inhibitory weights). Exceptions: For the simulations reported in Figures 12 and 18, the initial values of the inhibitory synaptic weights were 0.0 , thus preventing the inhibitory weights from changing during these simulations. This was done to simplify the graphs and to focus attention on the excitatory weights that were primarily responsible for the phenomena being manifested. Had the initial inhibitory synaptic weight values for Figures 12 and 18 been set at -0.1 , as was done for the other simulations, small changes in inhibitory weights would have been observed at some points in these simulations whereas the overall phenomena being manifested would have remained unchanged.

US (nonplastic) synaptic weight values: + 1.0 (excitatory weight) and $\mathbf{0 . 0}$ (inhibitory weight).

Lower bound on synaptic weights: $\left|w_{i}(t)\right| \geq 0.1$.

Neuronal output limits: $0.0 \leq y(t) \leq 1.0$

Neuronal threshold: $\theta=0.0$.

CS amplitudes (measured relative to zero-level baseline): 0.2 , except for Figure 7, where the amplitudes were 1.0, 0.5, and
0.25 , for $\mathrm{CS}_{1}, \mathrm{CS}_{2}, \mathrm{CS}_{3}$, respectively, and Figure 17, where the amplitudes were $0.2,0.2$, and 0.4 , for $\mathrm{CS}_{1}, \mathrm{CS}_{2}$, and $\mathrm{CS}_{3}$, respectively.

US amplitudes (measured relative to zero-level baseline): 0.5 , except for Figure 8, where the US amplitudes were 1.0, 0.5, and 0.25 for the USs occurring in conjunction with $\mathrm{CS}_{1}, \mathrm{CS}_{2}$, and $\mathrm{CS}_{3}$, respectively.

CS and US timing: See Table 1 for times of onset and offset of CSs and USs within a trial. Also specified in Table 1 are the trials during which each CS and US was present. For all of the CS-US configurations, the time of onset of the first stimulus was arbitrarily chosen to be 10 . Onset of a stimulus at time step $t$ means that the stimulus was on during time step $t$ and was not on during the preceding time step. Offset of a stimulus at time step $t$ means that the stimulus was off during time step $t$ and was not off during the preceding time step.

\section{Hebbian Model}

Where applicable, parameter values were the same as for the drive-reinforcement model, except that $c=0.5$, the initial synaptic weight values were 0.0 , and there was no lower bound on the synaptic weights.

\section{Sutton-Barto Model}

Where applicable, parameter values were the same as for the drive-reinforcement model, except that $c=0.5, \alpha=0.9$, the initial synaptic weight values were 0.0 , and there was no lower bound on the synaptic weights.

Table 1

Timing of the CS-US Configurations in Figures 4-20

\begin{tabular}{|c|c|c|c|c|}
\hline \multirow{2}{*}{$\begin{array}{c}\text { Figure } \\
\text { Number }\end{array}$} & \multicolumn{4}{|c|}{ CS and US Timing* } \\
\hline & $\mathrm{CS}_{\mathrm{i}}$ & $\mathrm{CS}_{2}$ & $\mathrm{CS}_{3}$ & US \\
\hline $4 a$ & $10 / 15 / 1-50$ & - & - & $14 / 15 / 1-50$ \\
\hline $4 \mathrm{~b}$ & $10 / 14 / 1-50$ & - & - & $14 / 15 / 1-50$ \\
\hline $4 c$ & $10 / 14 / 1-120$ & - & - & $14 / 15 / 1-120$ \\
\hline $5 a$ & $10 / 13 / 1-100$ & $20 / 24 / 1-100$ & $30 / 35 / 1-100$ & $\begin{array}{l}13 / 14 / 1-100 \\
23 / 24 / 1-100 \\
33 / 34 / 1-100\end{array}$ \\
\hline $5 b$ & $10 / 13 / 1-300$ & $20 / 24 / 1-300$ & $30 / 35 / 1-300$ & $\begin{array}{l}13 / 14 / 1-300 \\
23 / 24 / 1-300 \\
33 / 34 / 1-300\end{array}$ \\
\hline $5 c$ & $10 / 13 / 1-100$ & $20 / 24 / 1-100$ & $30 / 35 / 1-100$ & $\begin{array}{l}13 / 14 / 1-100 \\
23 / 24 / 1-100 \\
33 / 34 / 1-100\end{array}$ \\
\hline 6 & $4 / 5 / 1-50$ & $15 / 16 / 1-50$ & $28 / 29 / 1-50$ & $\begin{array}{l}5 / 6 / 1-50 \\
16 / 19 / 1-50 \\
29 / 34 / 1-50\end{array}$ \\
\hline 7 & $10 / 13 / 1-60$ & $20 / 23 / 1-60$ & $30 / 33 / 1-60$ & $\begin{array}{l}13 / 14 / 1-60 \\
23 / 24 / 1-60\end{array}$ \\
\hline 8 & $10 / 13 / 1-100$ & $20 / 23 / 1-100$ & $30 / 33 / 1-100$ & $\begin{array}{l}13 / 14 / 1-100 \\
23 / 24 / 1-100 \\
33 / 34 / 1-100\end{array}$ \\
\hline 9 & $\begin{array}{l}4 / 7 / 1-50 \\
14 / 17 / 1-50 \\
24 / 27 / 1-50 \\
34 / 37 / 1-50\end{array}$ & $\begin{array}{l}44 / 47 / 1-50 \\
54 / 57 / 1-50 \\
64 / 67 / 1-50 \\
74 / 77 / 1-50\end{array}$ & $\begin{array}{l}84 / 87 / 1-50 \\
94 / 97 / 1-50 \\
104 / 107 / 1-50 \\
114 / 117 / 1-50\end{array}$ & $\begin{array}{l}7 / 8 / 1-50 \\
17 / 18 / 1-50 \\
27 / 28 / 1-50 \\
37 / 38 / 1-50 \\
47 / 48 / 1-50 \\
67 / 68 / 1-50 \\
87 / 88 / 1-50\end{array}$ \\
\hline 10 & $10 / 13 / 1-120$ & $17 / 19 / 1-120$ & $24 / 25 / 1-120$ & $\begin{array}{l}13 / 14 / 1-120 \\
20 / 21 / 1-120 \\
27 / 28 / 1-120\end{array}$ \\
\hline 11 & $4 / 10 / 1-60$ & $13 / 20 / 1-60$ & $24 / 33 / 1-60 \dagger$ & $\begin{array}{l}4 / 10 / 1-60 \\
14 / 20 / 1-60\end{array}$ \\
\hline
\end{tabular}


Table 1 (Continued)

\begin{tabular}{|c|c|c|c|c|}
\hline \multirow{2}{*}{$\begin{array}{c}\text { Figure } \\
\text { Number }\end{array}$} & \multicolumn{4}{|c|}{ CS and US Timing* } \\
\hline & $\mathrm{CS}_{1}$ & $\mathrm{CS}_{2}$ & $\mathrm{CS}_{\mathbf{3}}$ & US \\
\hline & & & & $\begin{array}{l}27 / 33 / 1-60 \\
40 / 46 / 1-60 \\
57 / 63 / 1-60\end{array}$ \\
\hline 12 & $10 / 15 / 1-200$ & $7 / 12 / 61-200$ & - & $13 / 15 / 1-60$ \\
\hline 13 & $\begin{array}{l}10 / 13 / 1-300 \\
20 / 23 / 71-300\end{array}$ & $20 / 23 / 71-300$ & - & $13 / 16 / 1-200$ \\
\hline 14 & $10 / 13 / 1-200$ & - & - & $\begin{array}{l}13 / 16 / 1-70 \\
13 / 16 / 141-200\end{array}$ \\
\hline $15 \mathrm{a}$ & $10 / 14 / 1-25$ & - & - & $12 / 16 / 1-25$ \\
\hline $15 b$ & $10 / 14 / 1-25$ & - & - & $8 / 12 / 1-25$ \\
\hline 16 & $10 / 13 / 1-160$ & $10 / 13 / 101-160$ & & $13 / 14 / 1-160$ \\
\hline 17 & $10 / 13 / 1-50$ & $10 / 13 / 1-50$ & $10 / 13 / 1-50$ & $13 / 14 / 1-50$ \\
\hline $18 \mathrm{a}$ & $\begin{array}{l}10 / 12 / 1-80 \\
20 / 22 / 1-80\end{array}$ & $20 / 22 / 1-80$ & - & $\begin{array}{l}12 / 13 / 1-80 \\
22 / 23 / 1-80\end{array}$ \\
\hline $18 \mathrm{~b}$ & $\begin{array}{l}10 / 12 / 1-80 \\
20 / 22 / 1-80\end{array}$ & $20 / 22 / 1-80$ & - & $22 / 23 / 1-80$ \\
\hline $19 a$ & $10 / 12 / 1-100$ & $20 / 22 / 1-100$ & $\begin{array}{l}10 / 12 / 1-100 \\
20 / 22 / 1-100\end{array}$ & $12 / 13 / 1-100$ \\
\hline $19 b$ & $\begin{array}{l}5 / 7 / 1-20 \\
25 / 27 / 1-20\end{array}$ & $\begin{array}{l}15 / 17 / 1-20 \\
35 / 37 / 1-20\end{array}$ & $\begin{array}{l}5 / 7 / 1-20 \\
15 / 17 / 1-20 \\
25 / 27 / 1-20 \\
35 / 37 / 1-20\end{array}$ & $\begin{array}{l}7 / 8 / 1-20 \\
17 / 18 / 1-20\end{array}$ \\
\hline 20 & $10 / 13 / 1-160$ & $10 / 13 / 101-160$ & - & $13 / 14 / 1-160$ \\
\hline
\end{tabular}

*Time step of onset/time step of offset/trials during which stimulus was present. $† C S_{4}: 35 / 46 / 1-60 ;$ CS $_{3}$ : $51 / 63 / 1-60$

\section{GLOSSARY}

Mathematical symbols are defined in the text at the time they are introduced. In addition, for the convenience of the reader who wishes to review the definitions, they are listed below.

\begin{tabular}{ll} 
Symbol & \multicolumn{1}{c}{ Definition } \\
\hline$c$ & Learning rate constant. \\
$c_{j}$ & $\begin{array}{l}\text { Empirically established learning rate constant } \\
\text { proportional to the efficacy of classical delay con- } \\
\text { ditioning when the interstimulus interval is } j, \text { mea- }\end{array}$ \\
& $\begin{array}{l}\text { sured in discrete time steps. } \\
\text { Number of synapses impinging on a neuron. }\end{array}$
\end{tabular}

$s(t) \quad$ Sum of the weighted inputs to the neuron at time $t$; $s(t)$ can be viewed as an approximation to the neuronal membrane potential.

$t$

$w_{i}(t)$

$x_{i}(t) \quad$ A measure of the frequency of action potentials at the $i$ th synapse of a neuron at time $t$.

$\bar{x}_{i}(t) \quad$ Exponentially decaying trace of the presynaptic signal level $x_{i}$, equal to $\alpha \bar{x}_{i}(t-1)+x_{i}(t-1)$.

$y(t) \quad$ A measure of the postsynaptic frequency of firing of a neuron at time $t$. $y^{\prime}(t) \quad$ Maximal postsynaptic frequency of firing of a neuron.

$Y \quad$ Designates $y(t)$ for the last trial of a computer simulation.

$\alpha \quad$ Positive constant.

$\Delta s(t) \quad$ Change in the neuronal membrane potential at time $t$; i.e., $\Delta s(t)=s(t)-s(t-1)$.

$\Delta w_{i}(t) \quad$ Change in synaptic weight, $w_{i}(t)$, at time $t$, yielding the adjusted or new efficacy of the synapse at time $t+1$; i.e., $\Delta w_{i}(t)=w_{i}(t+1)-w_{i}(t)$.

$\Delta x_{i}(t) \quad$ Change in presynaptic signal level, $x_{i}(t)$, at time $t$; i.e., $\Delta x_{i}(t)=x_{i}(t)-x_{i}(t-1)$.

$\Delta y(t) \quad$ Change in postsynaptic signal level, $y(t)$, at time $t$; i.e., $\Delta y(t)=y(t)-y(t-1)$.

Neuronal threshold.

Longest interstimulus interval, measured in discrete time steps, over which classical delay conditioning is effective.
(Manuscript received October 19,1987; revision accepted for publication April 15, 1988.) 